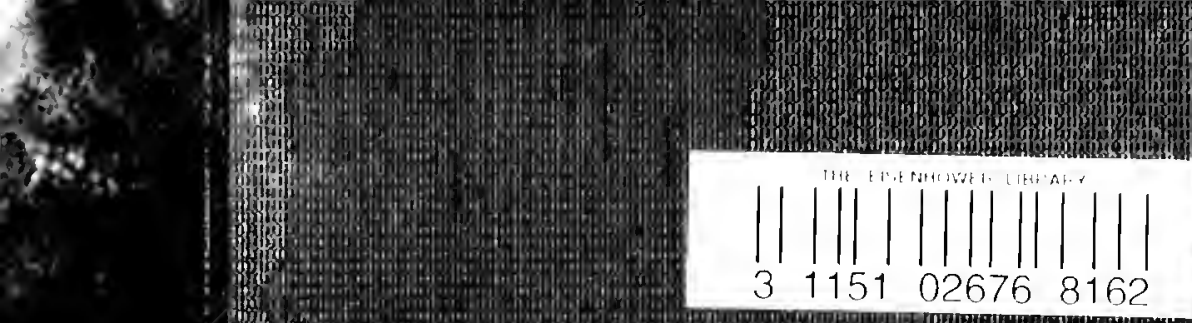

胲晾

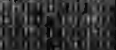
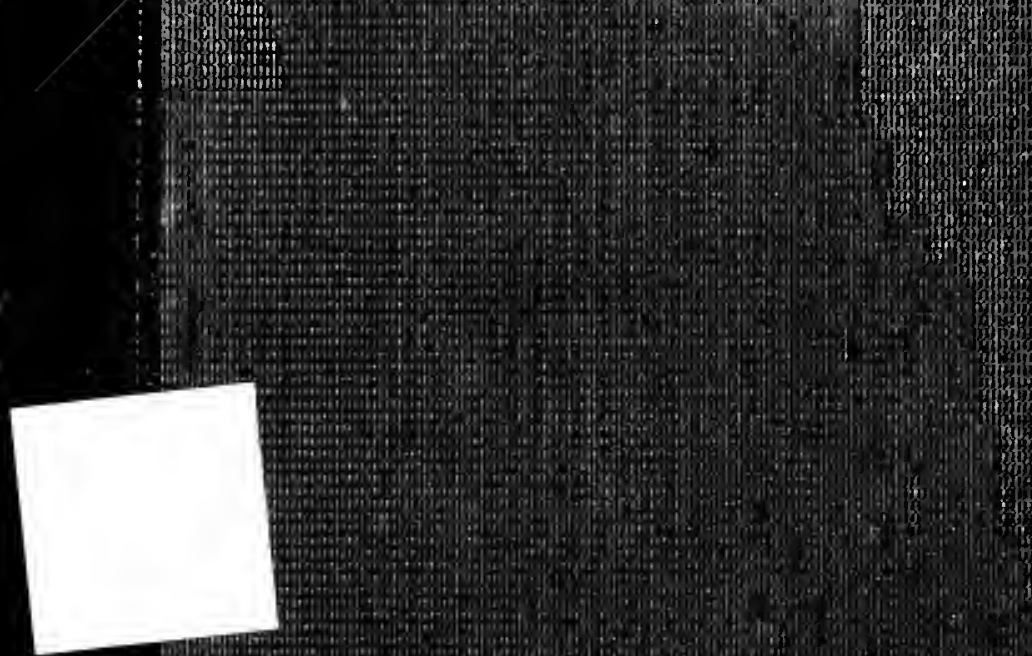

-

泡

(i)

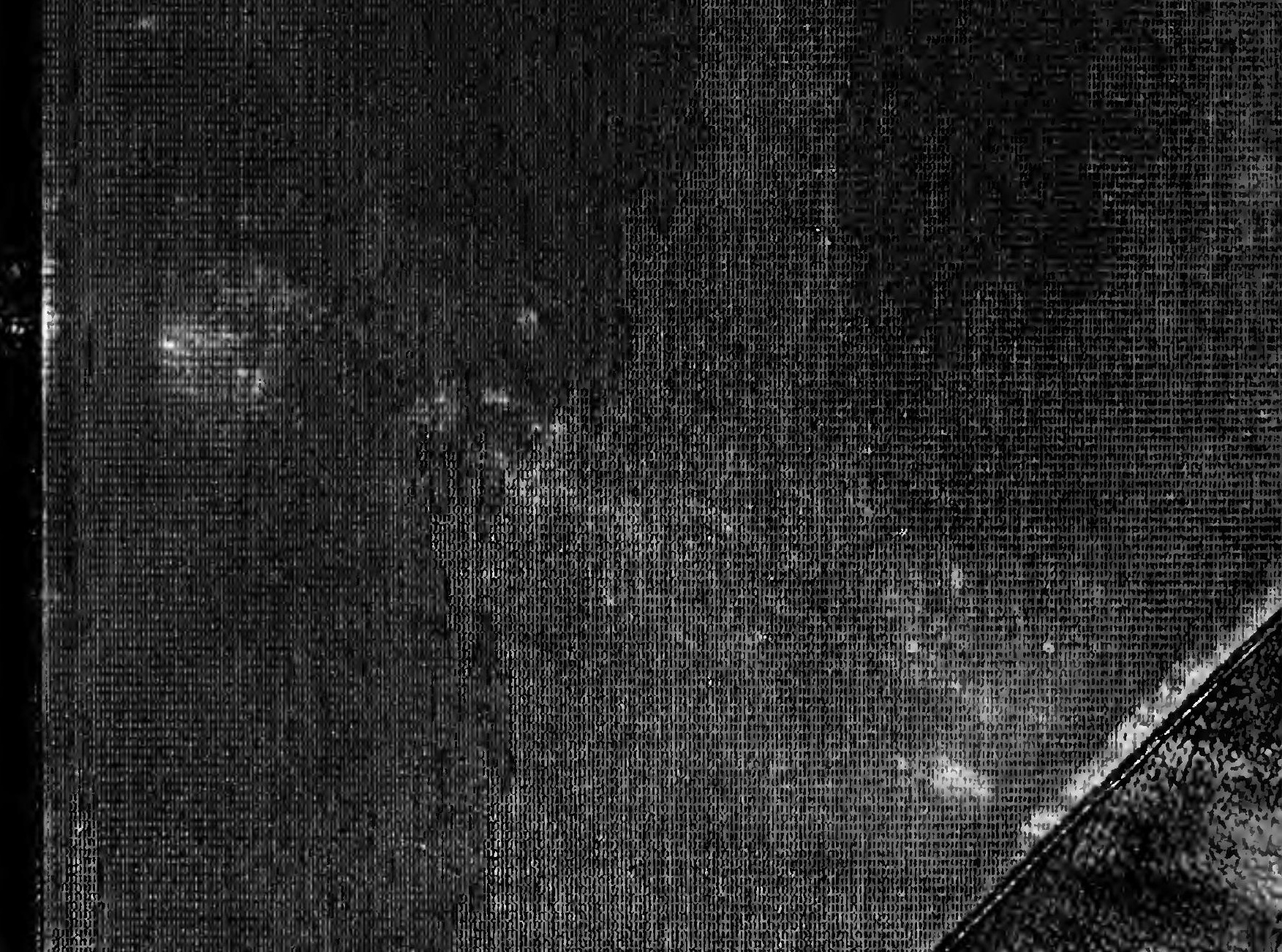

6
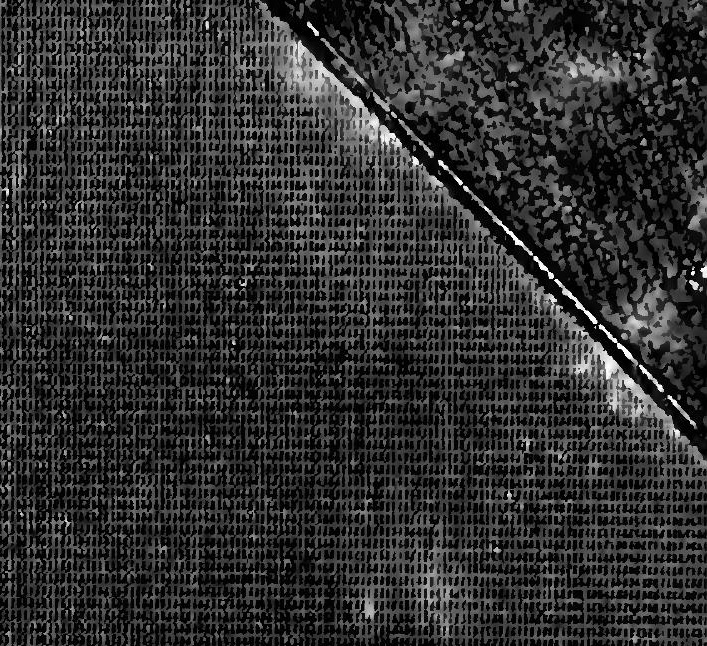

shy 


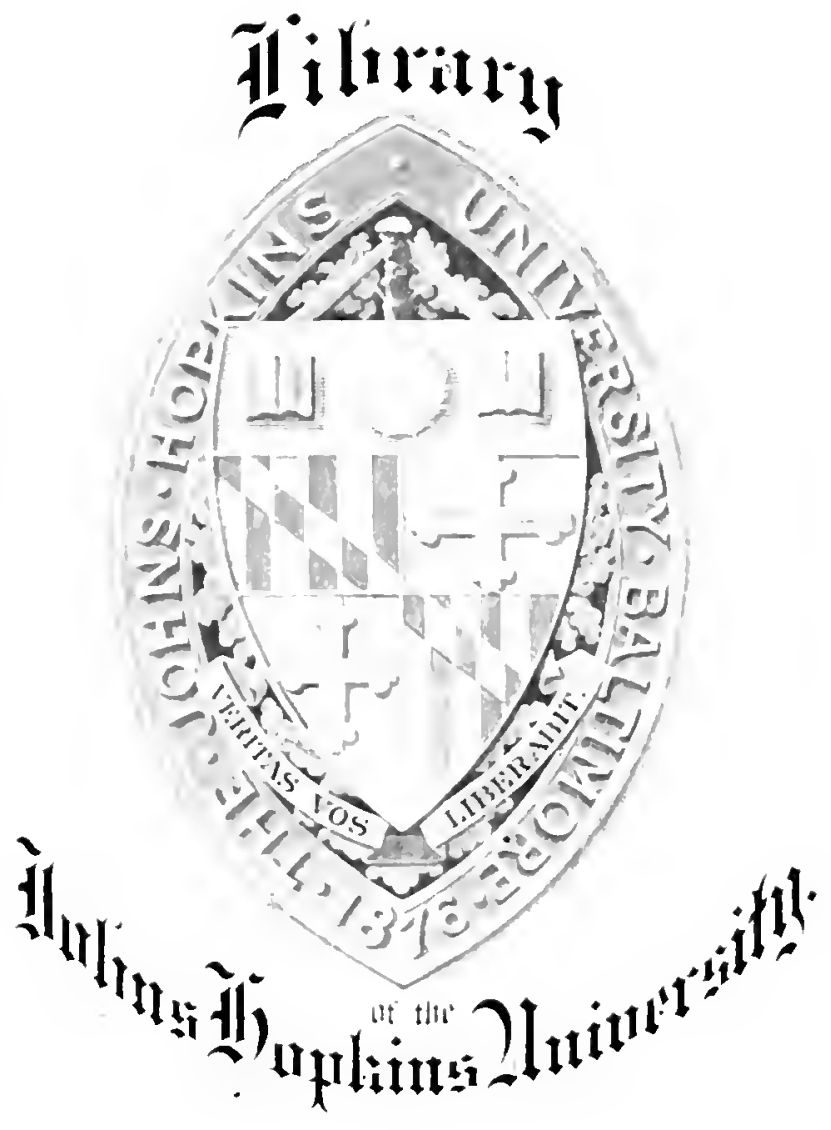




Ovectiqalioins in the 'Jane Y'uatie.

$\checkmark$ ?esen "?

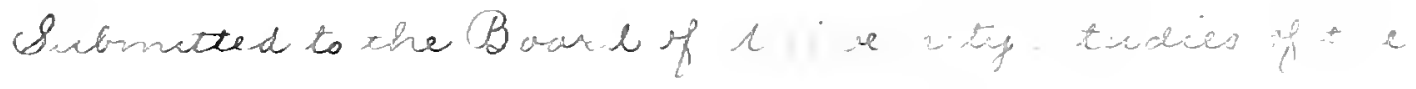

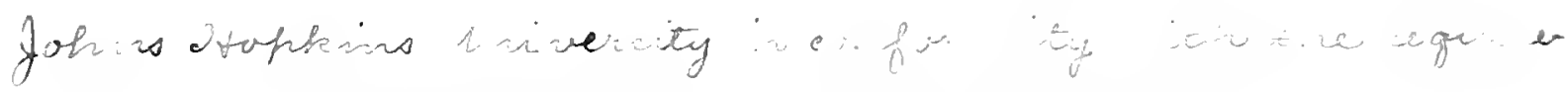
nents for the acoque if wetive of war

1913 
1. Introduction

Page I

2. The Undulato $~$

4

3. The Discrion ine ut of the Jessiar

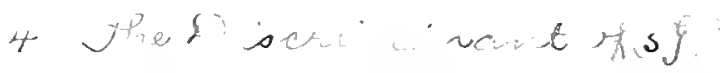

5. The Diacriminant of $t \xi,{ }^{6}$

6. The Pventy-une Liries

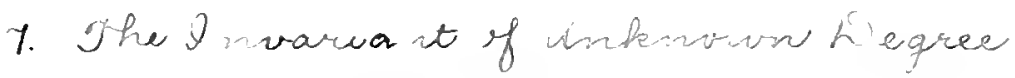

8. Bertain Nther $\mathcal{H}$ naria ito

19

9. The Eliminant of $S, T, H$.

25

10. The Eliminant of the Prlwo Poubie, Porrie, and Leine.

29

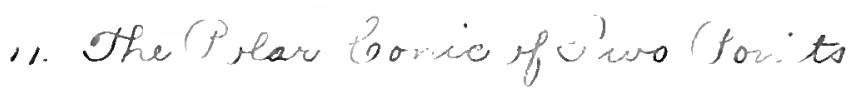

32

12. Sabrun's tounew

36

Srotes

49 

$\S 1$ Introduction.

The fume quartio(ax) ${ }^{4}$ is taken in the form

$$
\begin{gathered}
a x_{0}^{4} \\
+4 a x_{0}^{3} x_{1}+4 a_{2} x_{0}^{3} x_{2} \\
+6 h x_{0}^{2} x_{1}^{2}+12 l x_{0}^{2} x_{1} x_{2}+0 g x_{0}^{2} x_{2}^{2} \\
+4 b x_{0} x_{1}^{3}+12 m x_{0} x_{1}^{2} x_{2}+12 \pi x_{0} x_{1} x_{2}^{2}+4 c_{0} x_{0} x_{2}^{3} \\
+b x_{1}^{4}+4 b_{2} x_{1}^{3} x_{2}+6 f x_{1}^{2} x_{2}^{2}+4 e_{1} x_{1} x_{2}^{3}+c x_{2}{ }^{4}
\end{gathered}
$$

It w rel be convenient first to mentor briefly certain well-

R. wow forms connected with it that will be made use if w this article.

Of these several arise from the polar forms $(x)^{3}(\alpha y)$, $(\alpha x)^{2}(\alpha y)^{2},(\alpha x)(\alpha y)^{3}$. Since of each of these there is an $\infty^{2}$, the

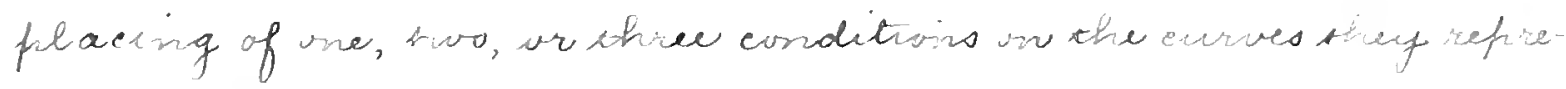
sent results, respectively, in a liens for therpole, $n$ a set $f$

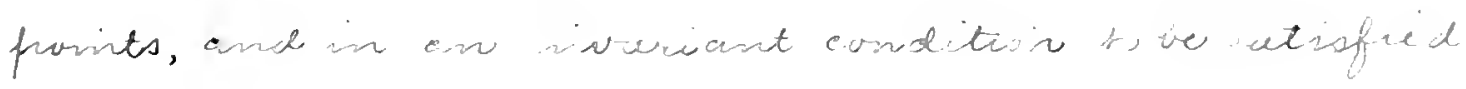
by the quartic.

Trons the polar line she r..ly conduit on peaceable is

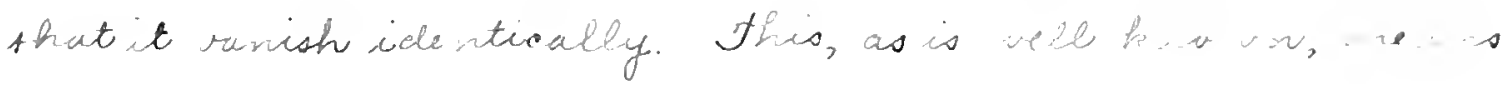



2

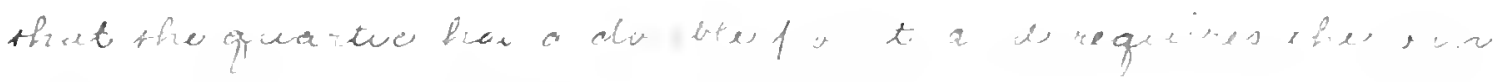

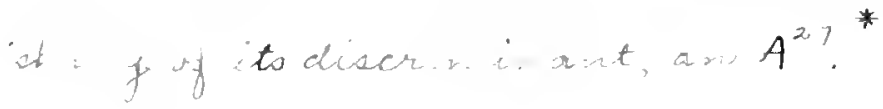

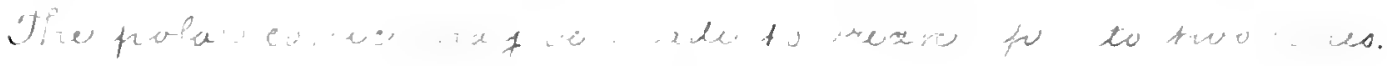

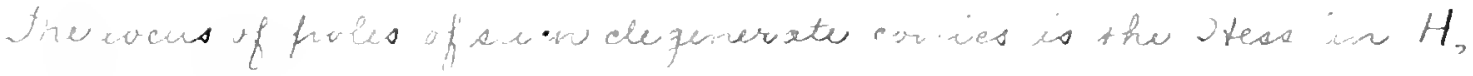

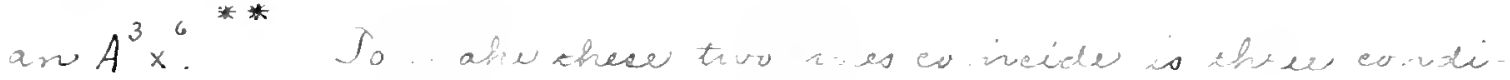

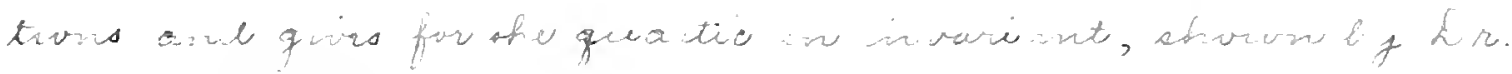
Thomene *** to be an $A^{48}$.

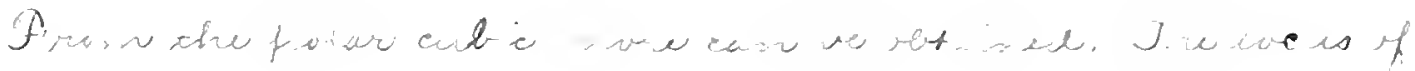

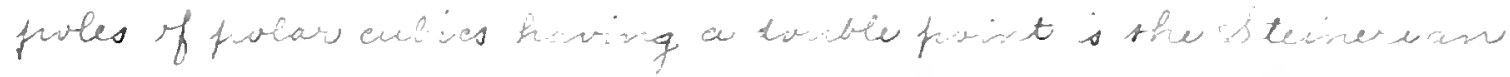

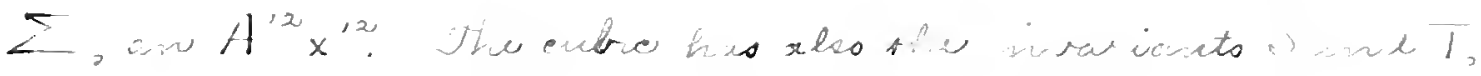

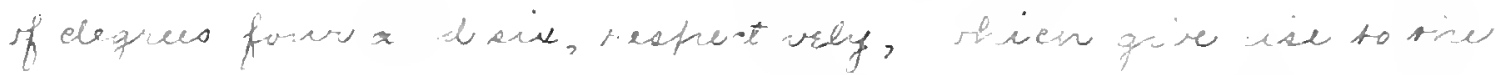

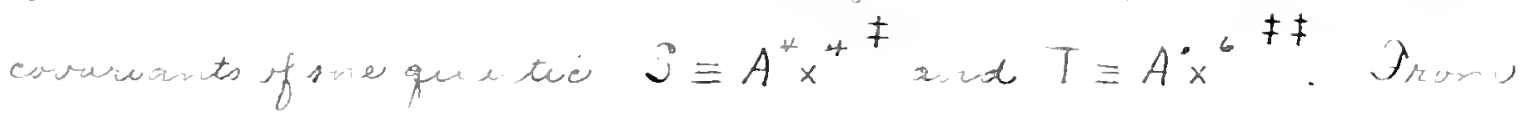

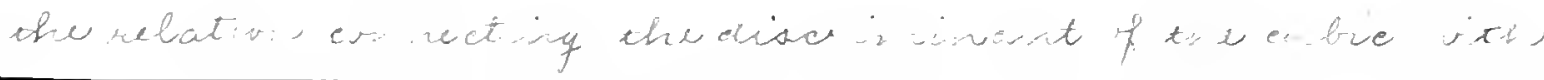
The otiatwe $A^{l} x^{\prime \prime} y^{\prime}$ is ceed to refiesent a ev vitat

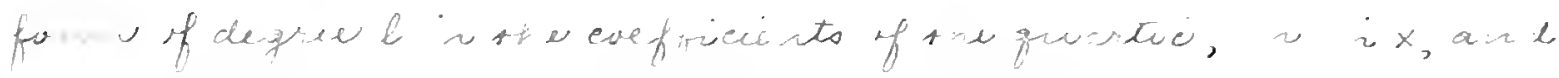
$2 \dot{\sim} \dot{y}$.

Seer to 1 .

Am. Gour. thath, wol XXXVIII, p.2, $24(916)$

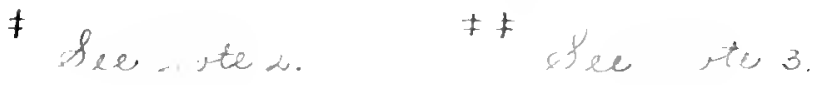



3

the tho where th we, ,

$$
2,+x^{3}+1^{2},
$$

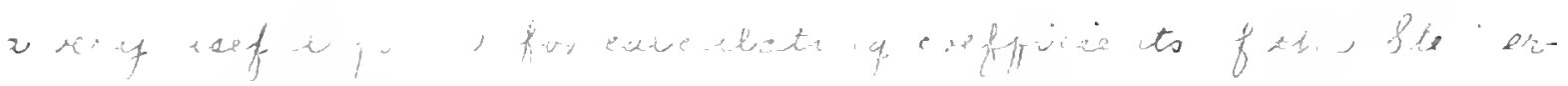

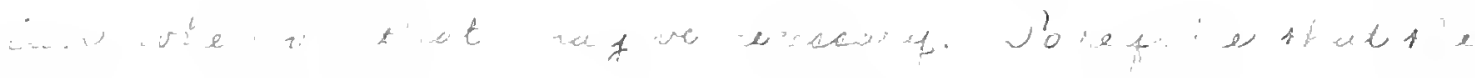

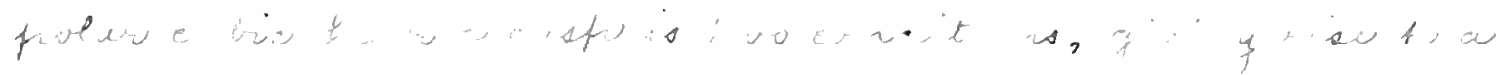

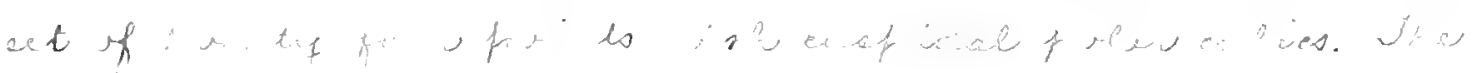

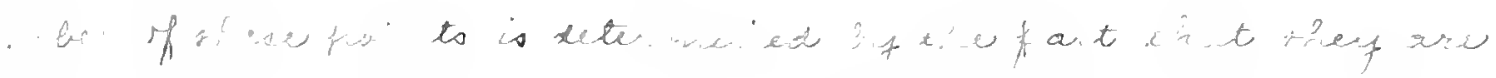

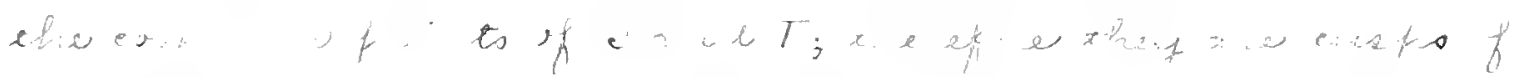

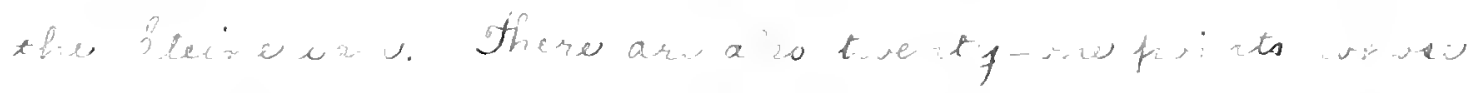

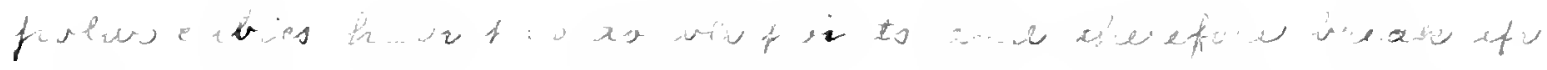

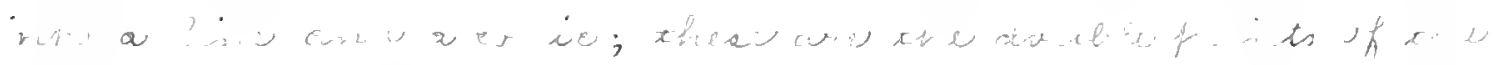

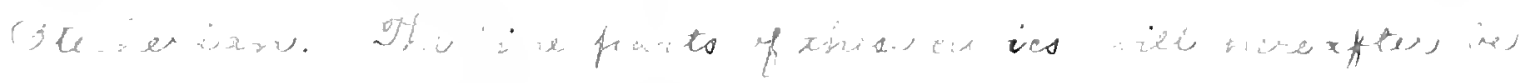

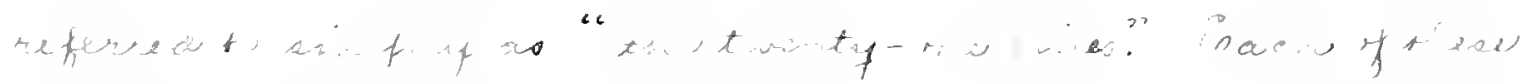

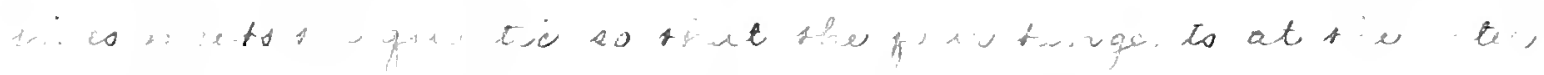

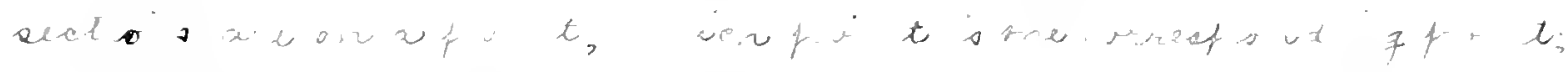

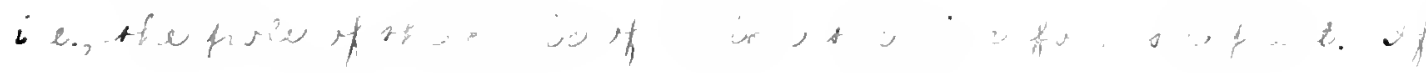

of $1,6,1,,+, \quad, \quad, *$

$$
v,=\nu=v=e,-\cdot
$$



$1 /$

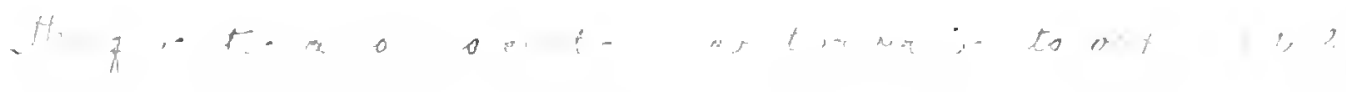

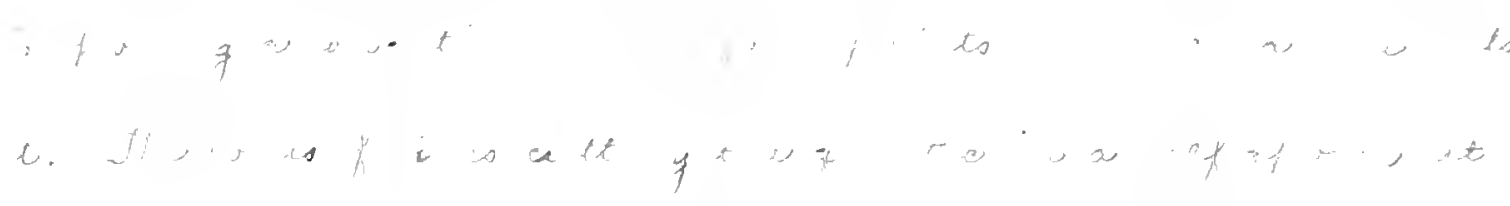

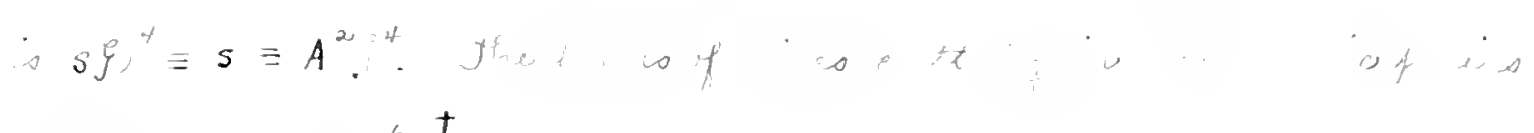
$\therefore t g^{\circ} \equiv t \equiv A^{3} p^{6} \cdot$

\$2. Utio lindulation.

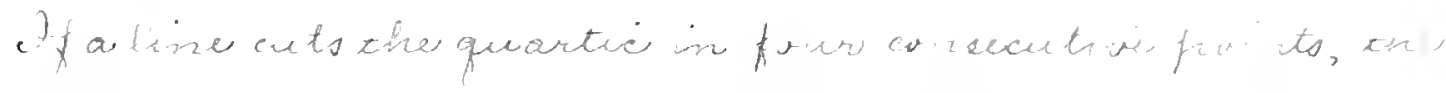

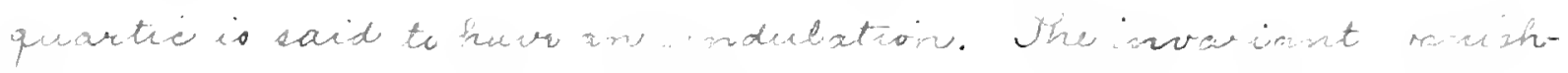

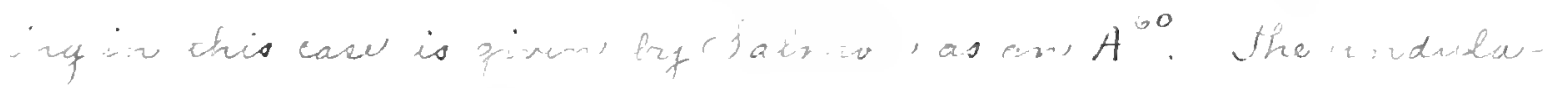

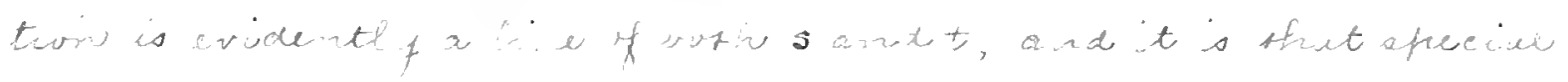

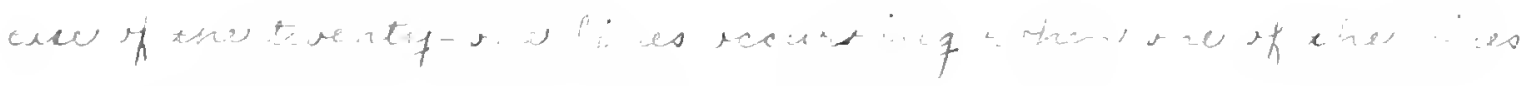

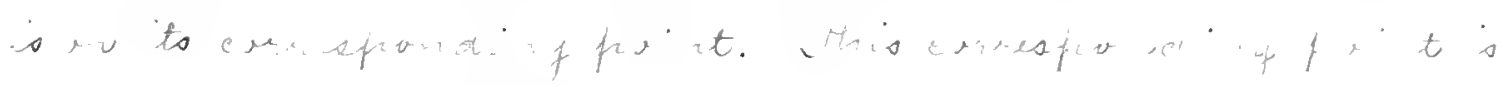

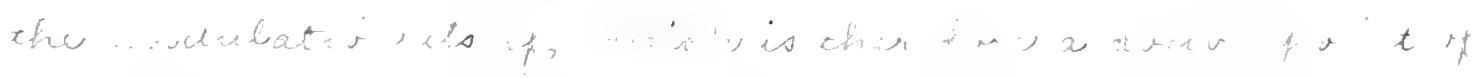

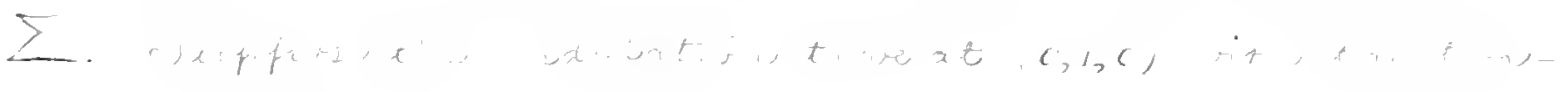
$+t x_{0} \cdot t$

$$
v=b_{2}+v_{1}=c
$$

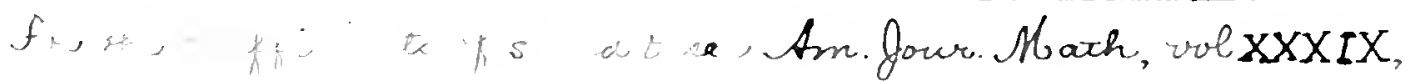
f. $232(1917)$. 

5

The anduclation is aleo a point of the ovessian, for to polar is ic is

$$
h x_{0}^{2}+2 b_{0} x_{0} x_{1}+2 n x_{0} x_{2}=x_{0}\left(h x_{1}+2 b_{1} x_{1}+2 n x_{2}\right),
$$

a fiai, rit thedor porite if s

is $(0,0,1)$, whech therefore becomes the correof wonding froint on the Steinerian. Then

$$
m=0 \text {. }
$$

The frolar enbic of $(0,1,0)$ is

$$
a_{1} x_{0}^{3}+3 h x_{1}^{2} x_{1}+3 l x_{0} x_{1}^{2}+3 b_{0} x_{0} x_{1}^{2}+3 \sim x_{0} x_{2}^{2} .
$$

This is made ufu of $x_{0}$ encl a eanie; to two double porinto are $\left(0, n, b_{0} r_{1}\right)$ and $\left(2, n,-b_{0} r_{1}\right)$; they are the twoforid to of the Ivessian carreaponding to the dowble tor it if the

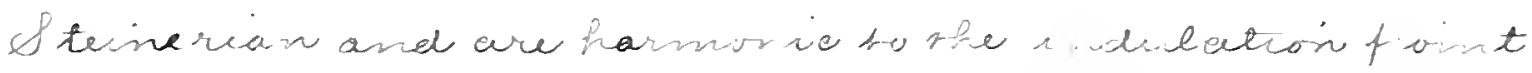
and the eleinerianforrt correster. ch. iq to the indulathoin considered as a Jessian fro t. She tems it th eontaining $x_{0}$ are

$$
x_{1}{ }^{4} x_{2}^{2} \cdot-e b_{0}^{2}+x_{1}^{2} x_{2}^{4}-2 c b_{0} w+x_{2}^{6} \cdot e v^{2}=-c x_{2}^{2}\left(b_{0} x_{1}^{2}+n x_{2}^{2}\right)^{2}
$$

Thirefirex 0 is a trifile tw rqent to the Jxesin. 

6

\$3. The Deccir natiof the Dessia U.

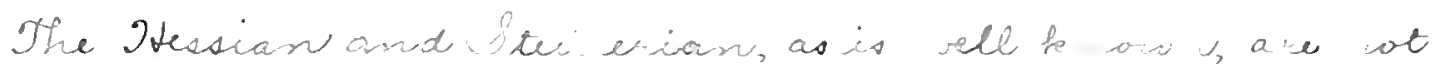

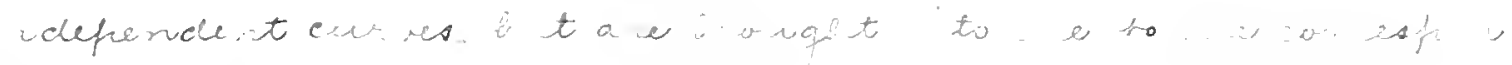
e vew by the relation

ous $\quad y)^{2} \alpha_{i}=0, \quad i=0,1,2$,

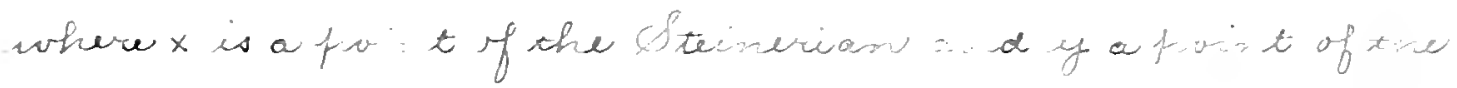

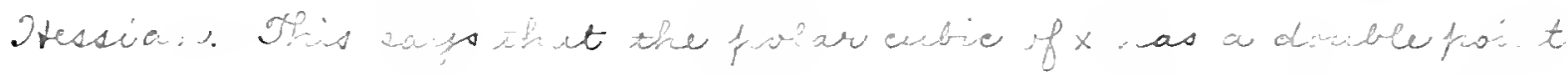

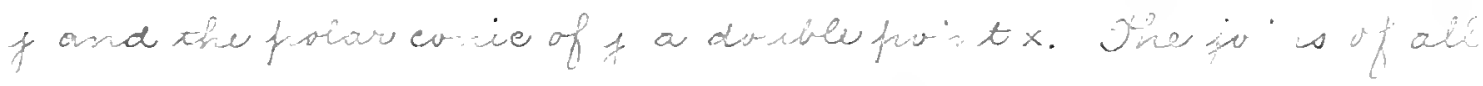

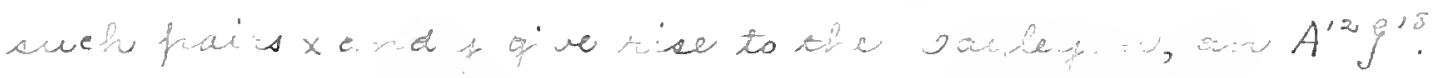

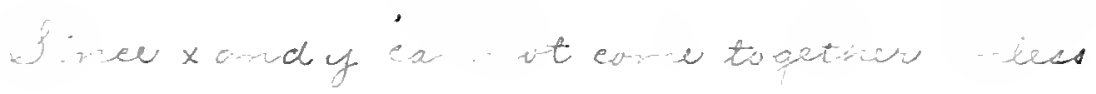

$$
\cos ^{3} \alpha_{1}-0, \quad i=0, s_{2},
$$

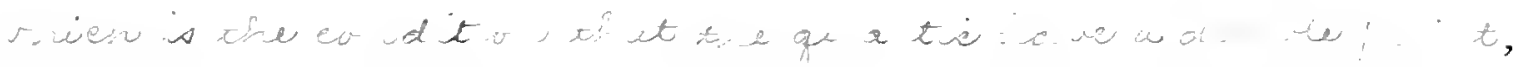

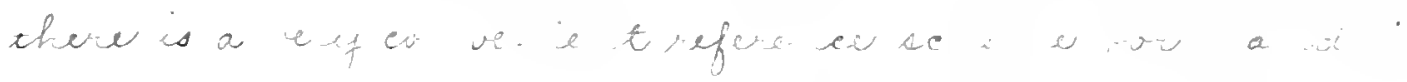

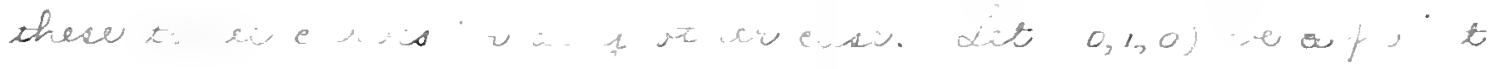

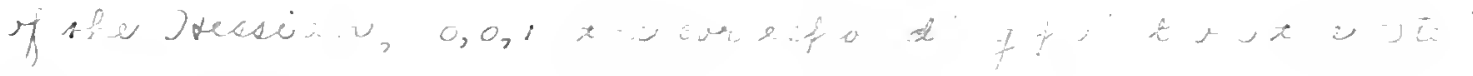

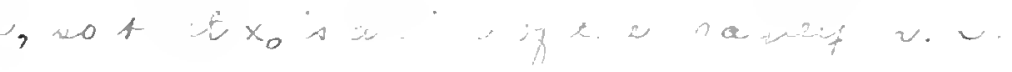

$$
\begin{aligned}
& x_{2} \alpha_{1}^{2} x_{i}=0, \quad \therefore=0, \alpha_{2} \\
& s=v_{2}=f=c .
\end{aligned}
$$




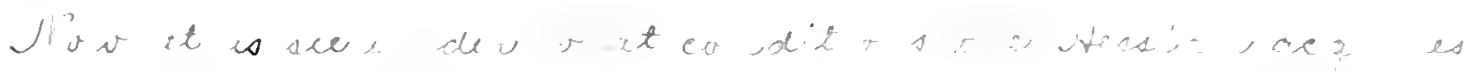

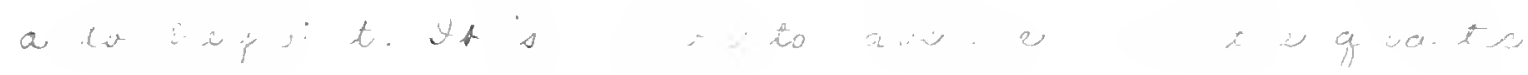

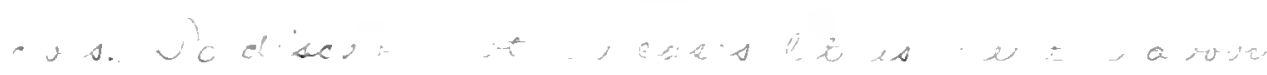

se. 2.

$$
\begin{aligned}
& H=x_{1}^{5} x_{0} \cdot 2\left(-2-v_{0}^{2}\right)+x_{1}^{5} x_{2} \cdot 2 e_{1}, v_{0}^{2} \\
& +\Delta, t x_{1} .
\end{aligned}
$$

Therefoi 0,10 is a wa visfor th

$$
\operatorname{tin}-v_{0}^{2}=0
$$

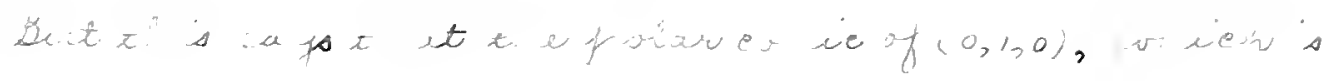

$$
x_{0}^{2}+2 b_{0} x_{0} x_{1}+b x_{1}^{2} \text {, }
$$

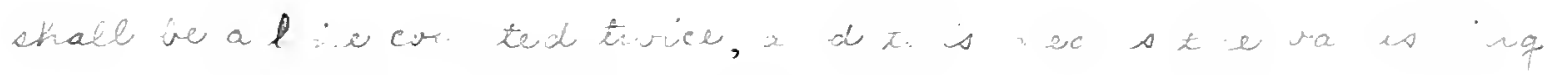

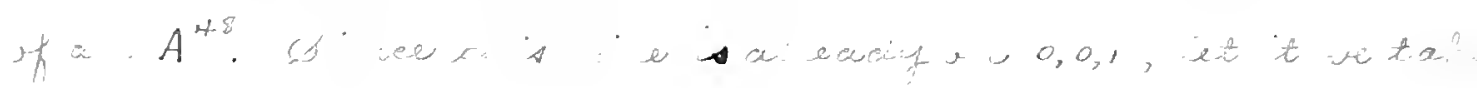
$\cos x_{0}=0$ that

$$
b_{0}=-c .
$$

ot. Wtef, e to tha fot $k, 0,1$ ) on $x_{1}$, namely.

$$
\begin{gathered}
\left(a k+a_{2}\right) x_{0}^{3}+3\left(a_{1} k+l x_{0}^{2} x_{1}+3\left(a_{2} k+q\right) x_{0}^{2} x_{2}+0(l k+\cdots) x_{0} x_{1} x_{2}\right. \\
+3\left(q k+c_{0}\right) x_{0} x_{2}^{2}+3\left(k+c_{1}\right) x_{1} x_{2}^{2}+\left(c_{0} k+c\right) x_{2}^{3},
\end{gathered}
$$

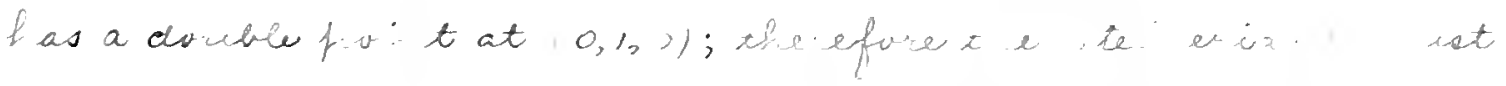

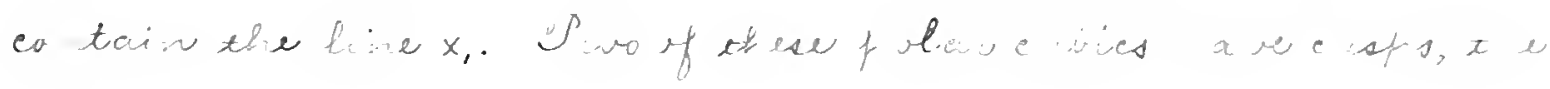





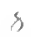

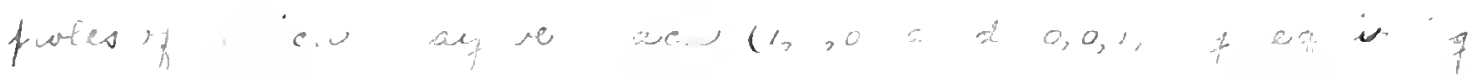

$-t$

$\omega \quad t=0$.

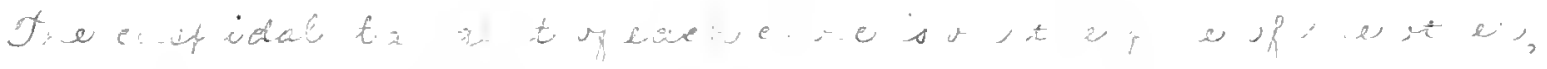

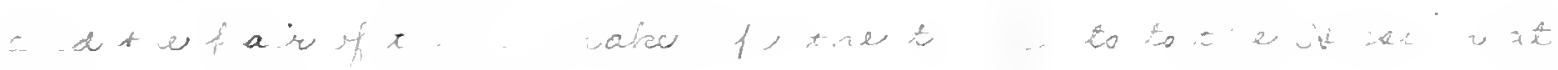
to no $i x+\cdots$, t. $j$.

$$
\begin{aligned}
j= & -a_{1}^{2} c_{1}^{2} x_{0} x_{2}^{2} \\
& +x_{1} L_{0}^{3}\left(-b a_{1} a_{2} c_{0}+b a_{1} q^{2}\right)+\quad+x_{2}^{3}\left(-b a_{2} c_{0} c_{1}+b c_{1} q^{2}\right) ? \\
& + \text { igher bo no in, }
\end{aligned}
$$

a in

$$
\begin{aligned}
T= & 8 a_{1}^{3} c_{1}^{3} x_{0}^{3} x_{2}^{3} \\
& +x_{1} \mid x_{0}^{5} \cdot 4 b a_{1}^{3} c_{0}^{2}+\cdots+x_{2}^{5} \cdot 4 b a_{2}^{2} c_{1}^{3} \\
& + \text { iqgen bc s s i } x_{1} .
\end{aligned}
$$

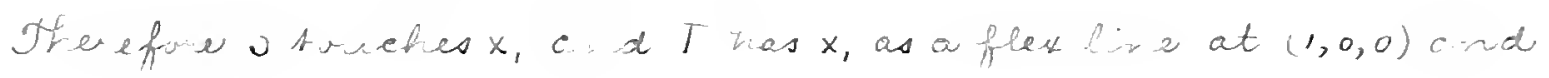
$(0,0,1)$. Alo

$$
\begin{aligned}
& Z=x_{1} x_{0}^{8} x_{2}^{3} \cdot 04 a_{1} c_{0}^{2} c_{1}^{3}+\quad+x_{0}^{3} x_{2}^{8} \cdot 4 \cdot 4 a_{1}^{3} a_{2}^{2} c_{1}^{6}
\end{aligned}
$$

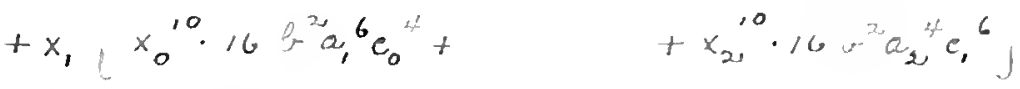

$$
\begin{aligned}
& +\quad i \neq n \text { be s } \sim x_{1}, \text {, }
\end{aligned}
$$

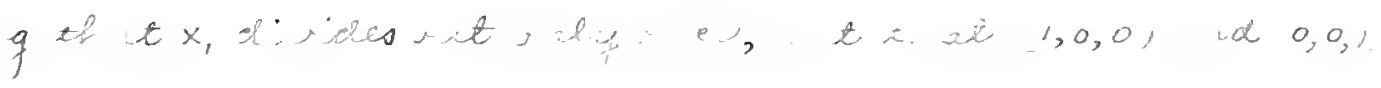



7

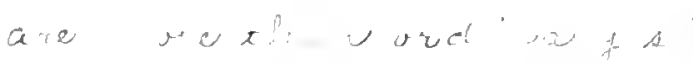

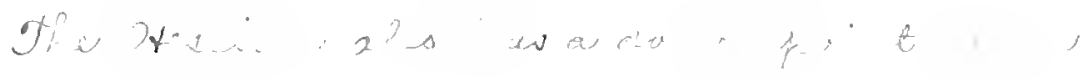
$c_{1}, \cdots c$

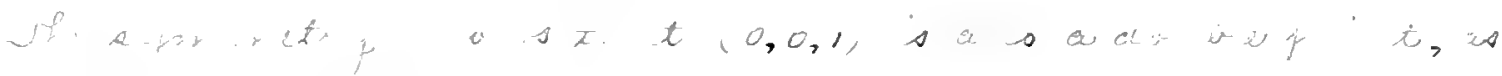

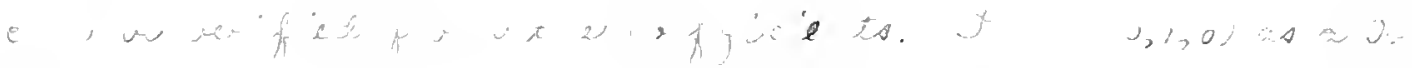

,$v \cdot t \cdot a j(0,0,1)$ us

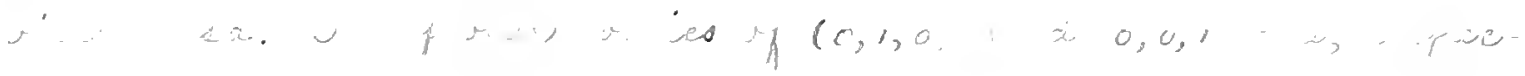

$f \cdot f$

$h x_{0}^{s}+2 v_{0} x_{0} x_{1}+\cdots$,

$=\lambda \geqslant x_{0}+\alpha c_{0} x_{0} x_{1}+\cos _{2}^{2}$.

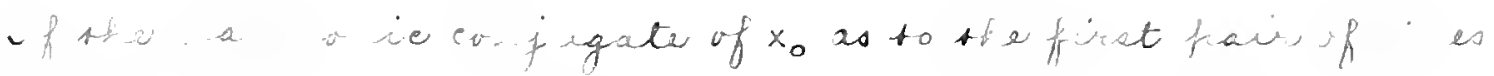

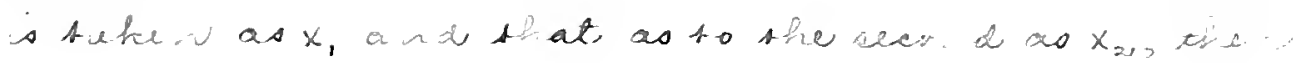

$$
b_{0}=c_{0}=0
$$

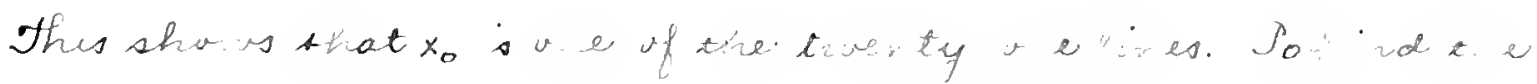

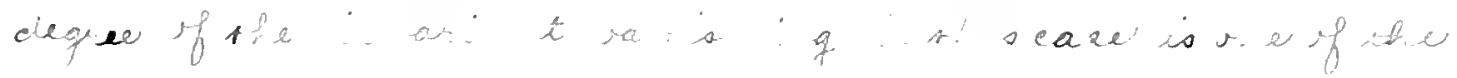
vejiets of of a tigato.

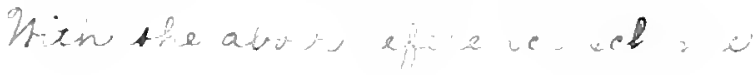

$$
\begin{aligned}
& j=x_{1} x_{2}^{3} \cdot-\sec
\end{aligned}
$$

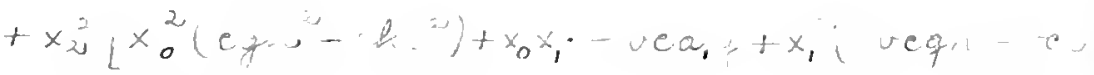

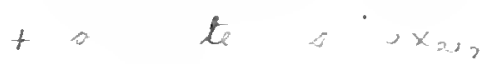



10

$$
\begin{aligned}
& T=x_{1} x_{2}^{5} \cdot 2 b c^{x} l^{3} \\
& +x_{2}^{4} \mid x_{0}^{2} \cdot 3 e^{2} b^{2} l^{2}+2 x_{0} x_{1}\left(-3 b e^{2} a_{2} k b+\cdots c^{2} a_{1}\right)^{2},
\end{aligned}
$$

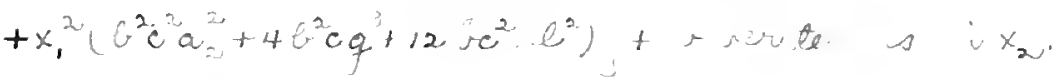

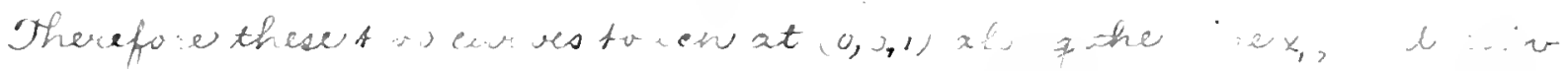

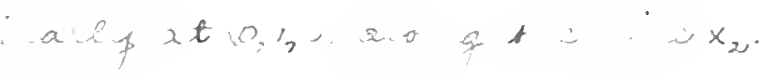

$$
\begin{aligned}
& E=x_{1}^{2} x_{2}^{10}+v^{2} c^{4} t^{6} \\
& +x_{20}^{a^{r}} x_{0}^{2} x_{1}-12 v e^{4 t} e^{2} e^{2}+x_{0} x_{1}^{2} \cdot r^{2} e^{3}\left(-3-c^{2} a_{2} u+g c^{2} a_{1} c^{2}\right)
\end{aligned}
$$

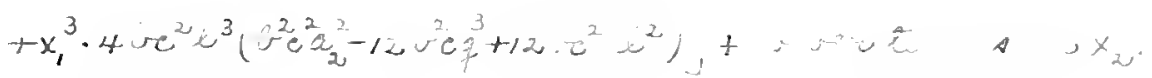

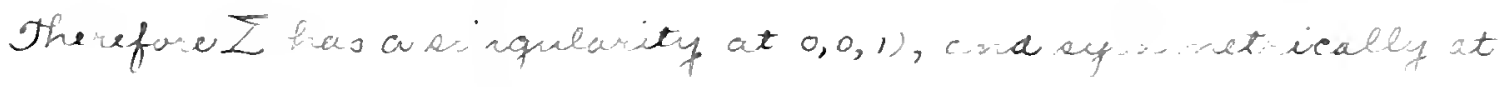

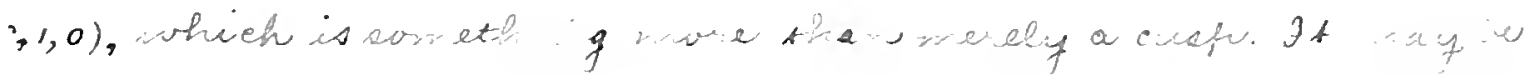
$x$ tac-node.

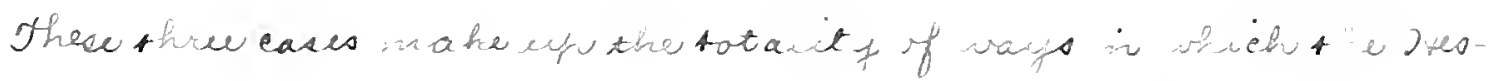

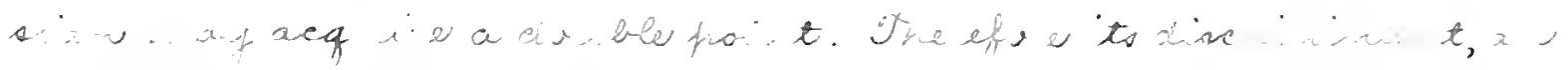

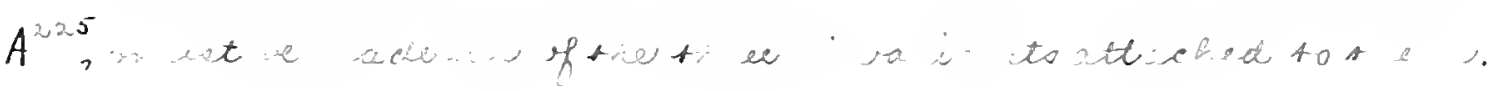

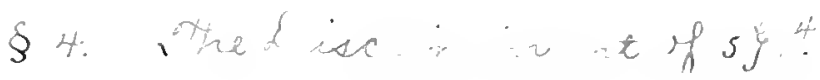

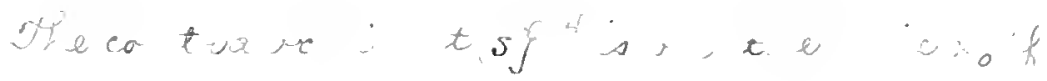

$$
b_{-}=k=e-c .
$$



the proint of eontact is gave'ru $b y$

$$
3 c_{1}, m g_{1}+\left(-b c_{0}+b_{0} e_{1}\right) \xi_{2}, 0 .
$$

Sincer $\left(0,0,1\right.$, is takerv as a if wa e of the frotits in mueh, $x_{0}$ cuts

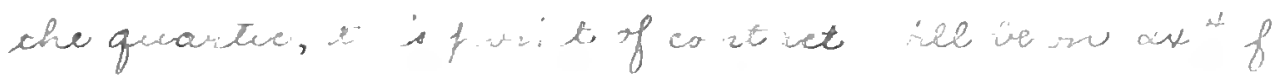

$$
i=0 .
$$

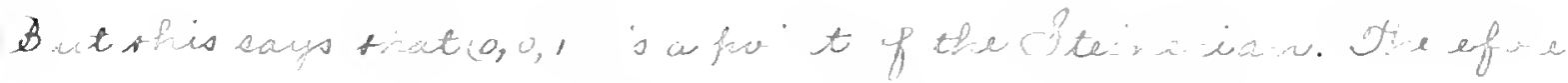

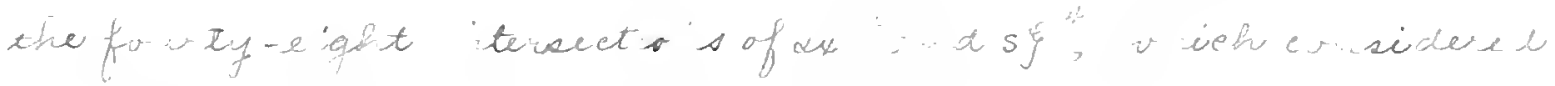

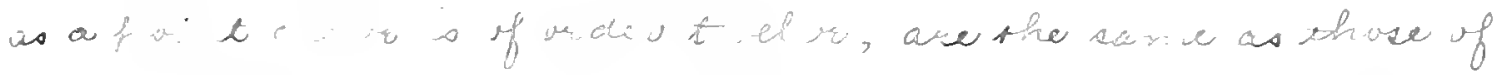

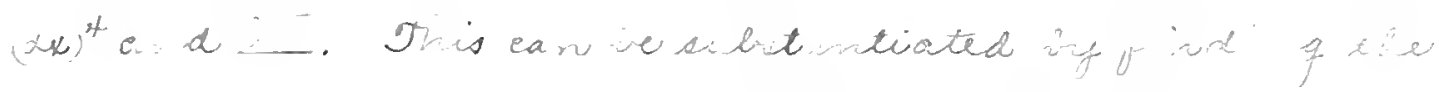

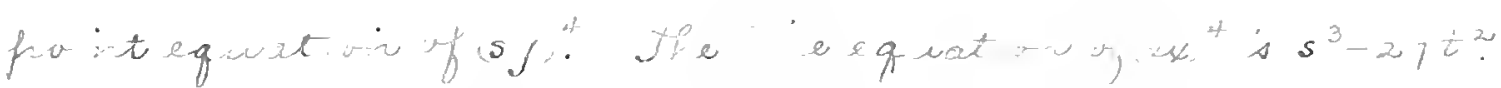

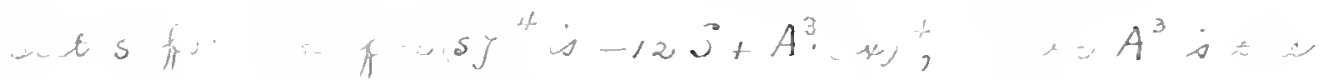

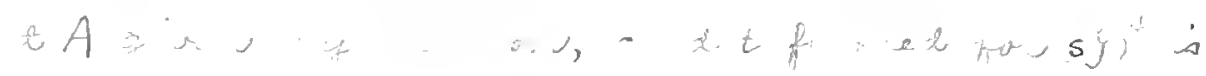

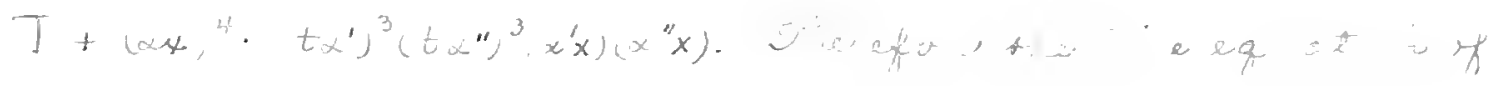
$s\}$

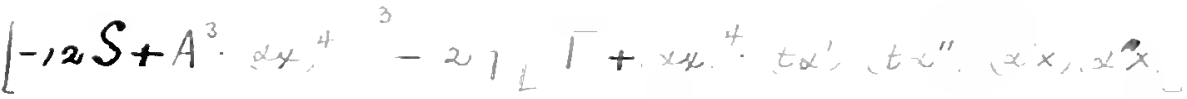

$$
\begin{aligned}
& =-27 \bar{Z}+(\alpha x)^{4} \cdot A^{\prime \prime} x^{8} .
\end{aligned}
$$

$x_{0}$ will hecome a double line of s or ly' $f$

$$
b_{0}=c_{0}=m=0,
$$

If $c_{1}=0$, the quantie we che hav $a$ welat $\omega$. 

2

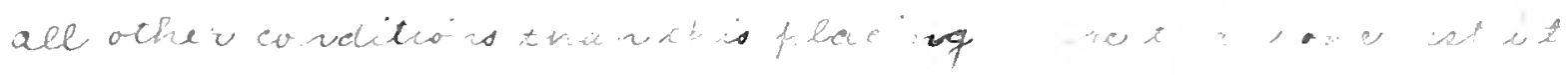
iort sow the gwatie. She fro ito in which $x_{0}$ macts the quar. tié cere $0,0,1),(0, k, 1), 0,0 k, 1),\left(0,0^{2} k, 1\right.$, whe $k^{3}-4 \cdot e^{-1}$ and $v^{3}=1$. The tarrgents to the quar eve at tien forit fo to are, ectertielf-

$$
\begin{gathered}
e_{1} x_{1}=0, \\
3 n k x_{0}-3 c_{1} x_{1}+3 c_{1} k x_{2}=0, \\
3 n \omega k x_{0}-3 c_{1} x_{1}+3 c_{1} \nu k x_{2}=0, \\
3 n \omega^{2} k x_{0}-3 c_{1} x_{1}+3 c_{1} \omega^{2} k x_{2} o_{1}
\end{gathered}
$$

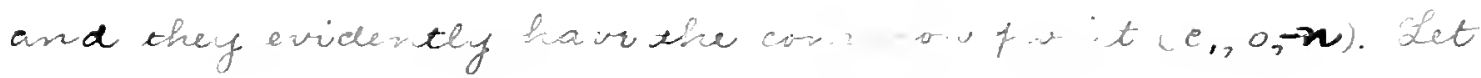

$$
\imath=0 \text {, }
$$

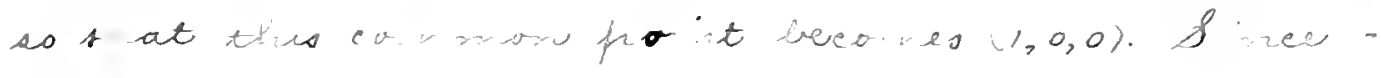

$q$

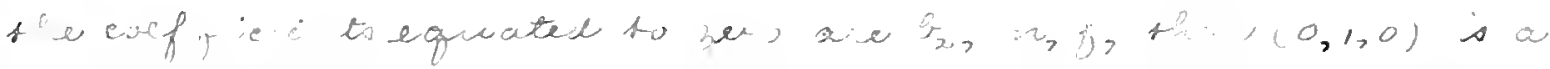

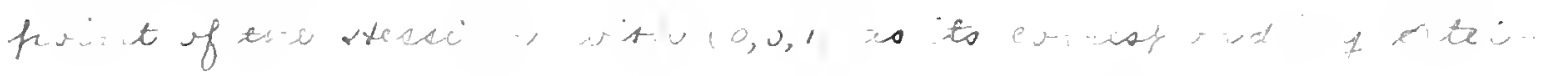

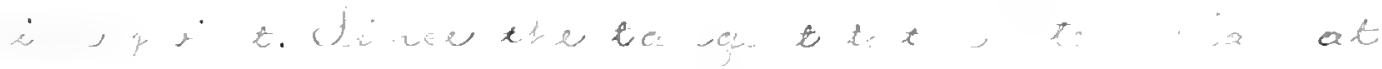

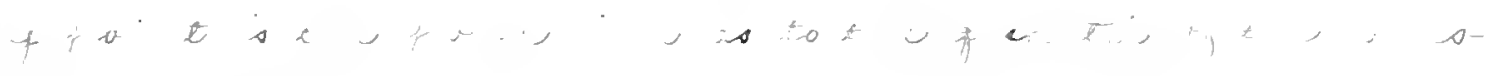

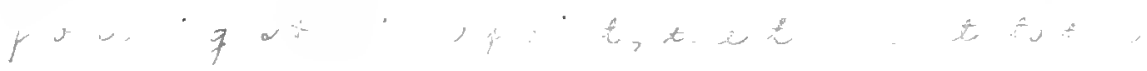

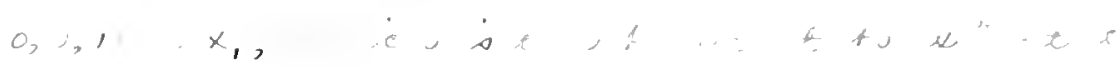

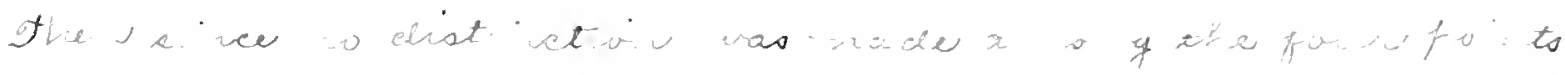



3

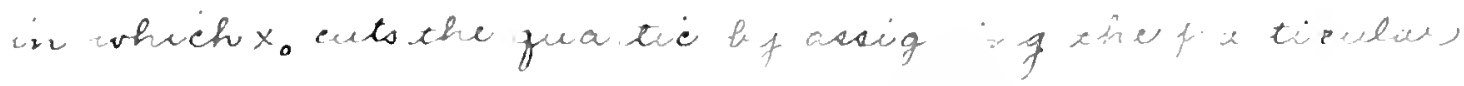

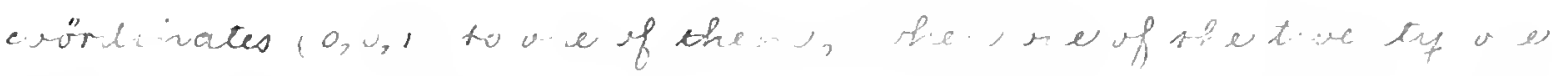

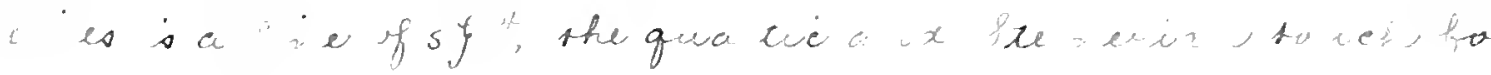
$t=$ ies vo this $f^{\prime}$, the four tangents going thwe the earresponding fwint. Hlso, since the tangent to the Dsescisu at $(0,1,0)$ is $x_{2}$, the tangento at the foris Dtessicin fovi, to conres-

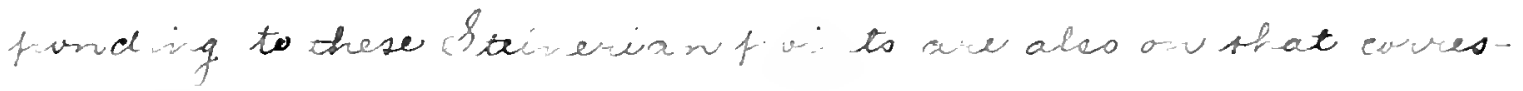
frow if wit.

$$
\begin{array}{r}
x_{0} \text { will also be a } l \text { of } s y^{4} f \\
b=b_{2}=f=0 ;
\end{array}
$$

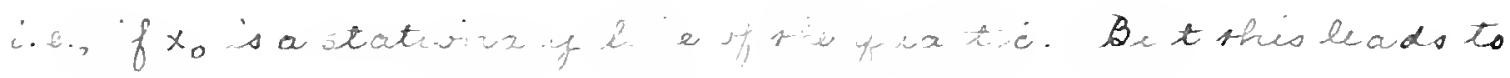

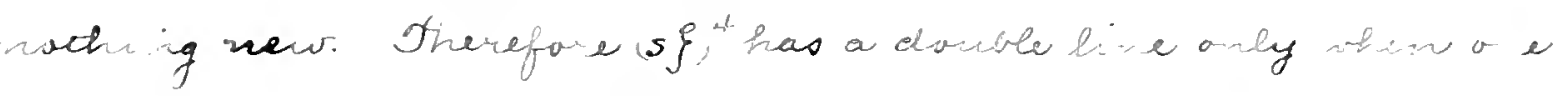

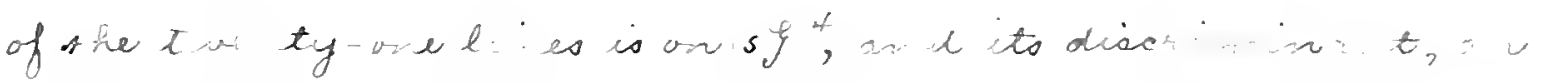
$A^{54}$, expresess this evaditain.

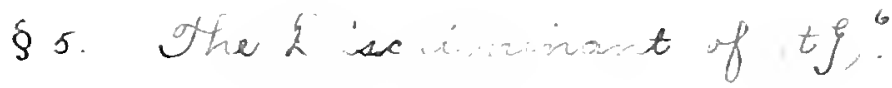

The contravareant $(t f)^{6}$ is on the hilex. $f$

$$
b=b_{2}=f=0 \text {. }
$$



Then

$$
t \xi^{\circ}=2 \xi_{0}^{5} \xi_{1} \cdot 2 b_{0} e_{1}^{2}+t_{0} \text { er powers in } \xi_{0} .
$$

$x_{0}$ becomes a double tine either if ${ }_{n}=0$, which sa go the the

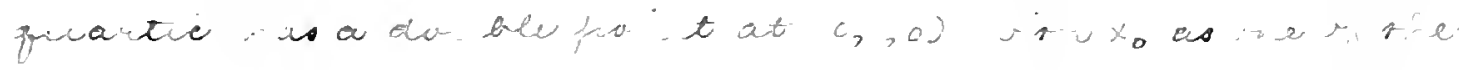

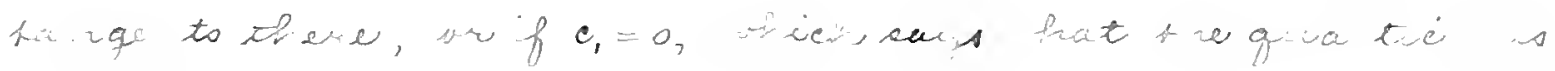
$\therefore 3$ - she tot

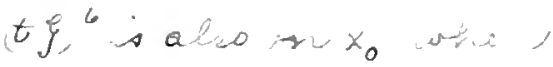

$$
\begin{aligned}
& b_{2}=k=e_{1}=0 .
\end{aligned}
$$

Dire,

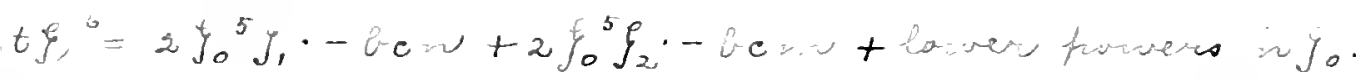

For $x_{0}$ to be a dis be time requires either $b=j$ or $c=0$, whines refeats the undulation condition, of $m=n=0$, which is the condition on the qua tie when when the ore sian nus

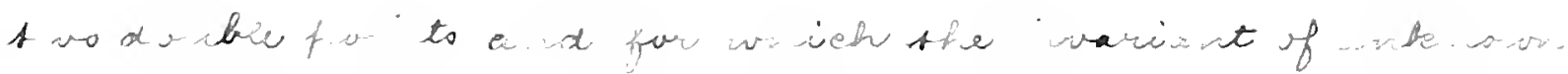

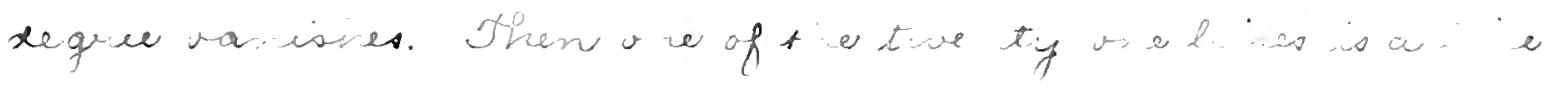
if $t \rho:$ :

ty) also has to as a line when

$$
b=f=c-?,
$$

but this leads to wet ow Striction che discriminant 

13

of $\left\{\begin{array}{c}G, 5 \\ ,\end{array}\right.$

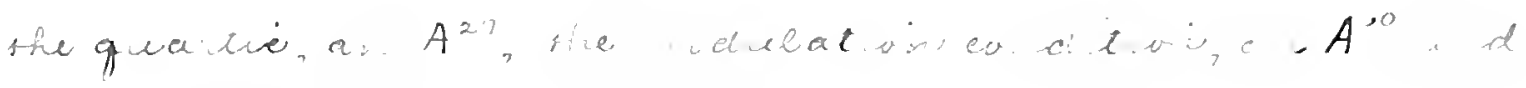

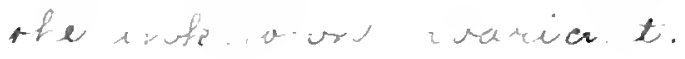

$\S$ o. The Owenty-une Lines.

The twenty-one ines " are given by an $A^{i} x^{2 \prime}$, where is

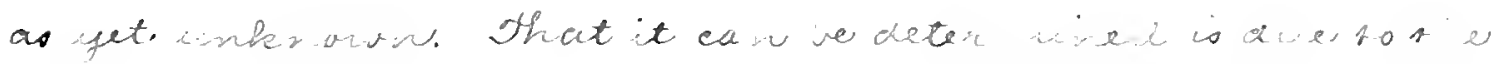

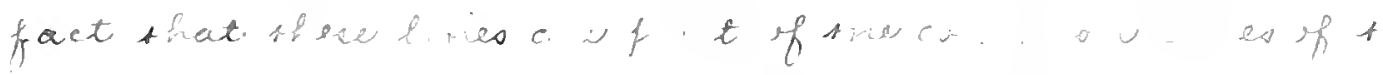

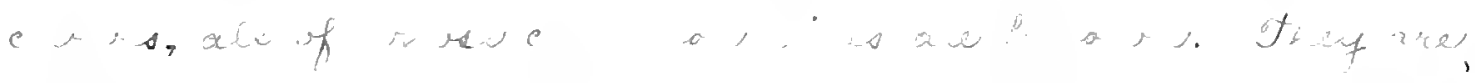

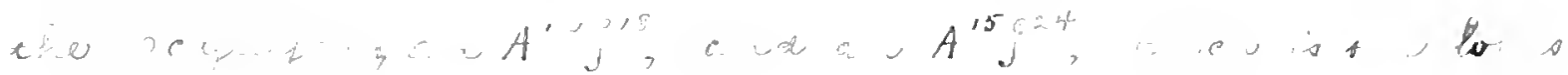

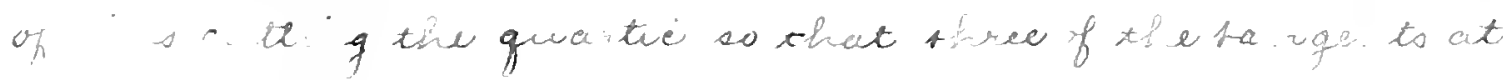

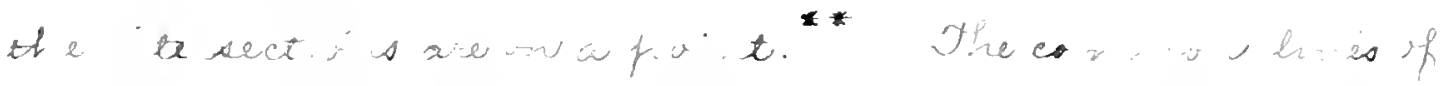

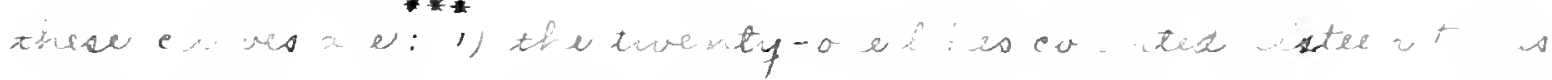

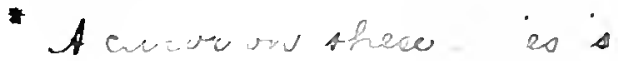

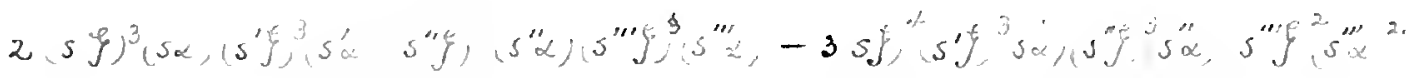

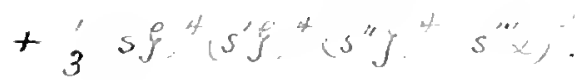

* Am. Jour. Hooch, vol. XXXIX, p.22, (19,7).

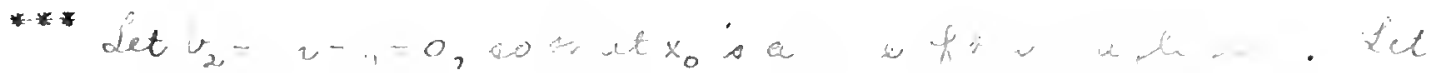



16

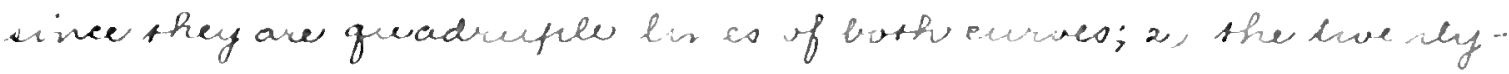

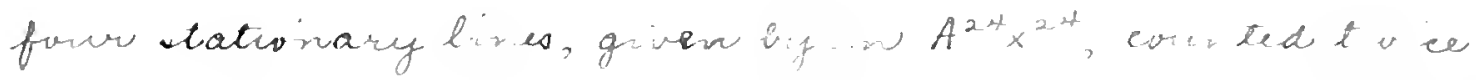

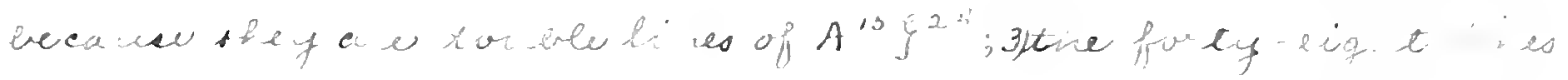

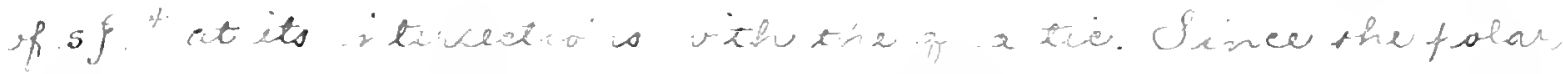

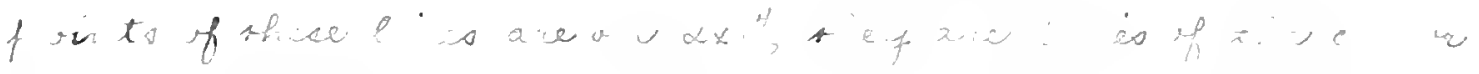

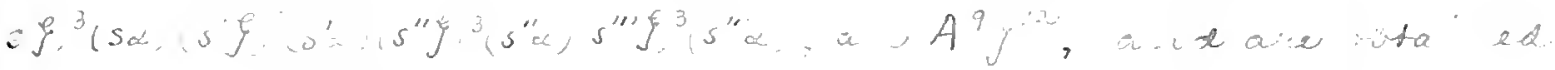

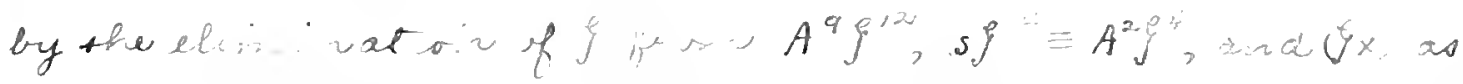

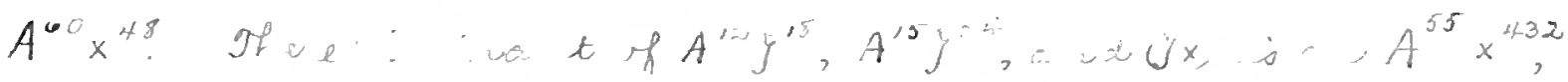

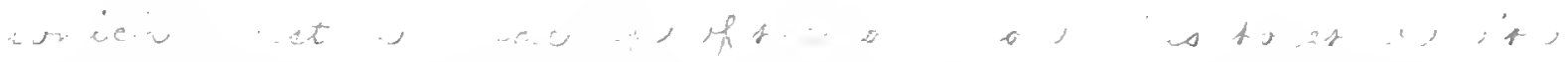

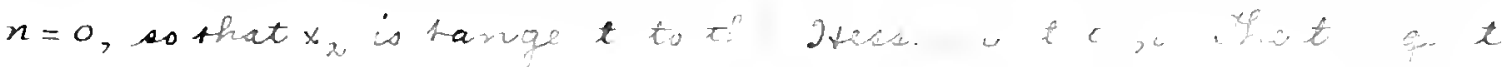

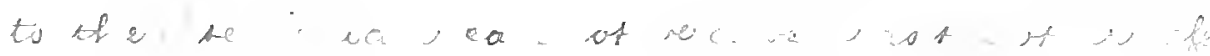

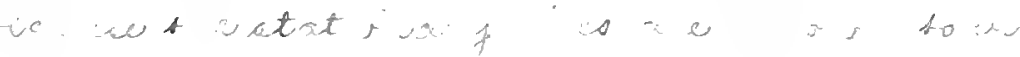

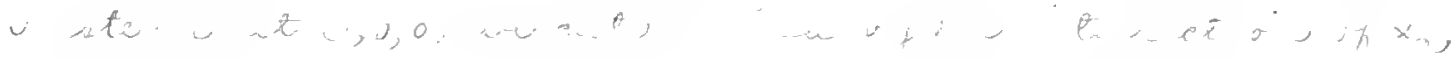

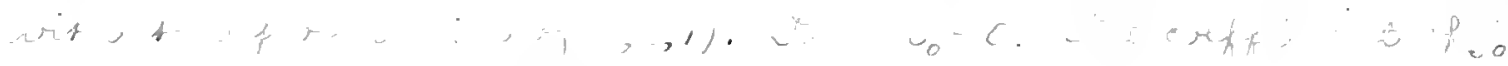
i $A^{15} f^{24}$ wecones $\frac{13}{3} b^{2} e b, e_{1}^{8} . I_{t} b=0$, $x_{0}$ is a atativa y 2

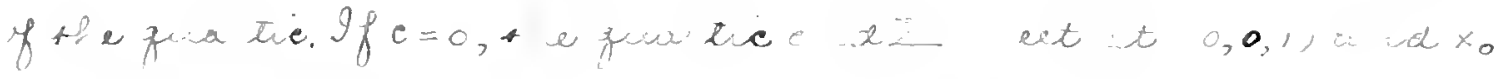

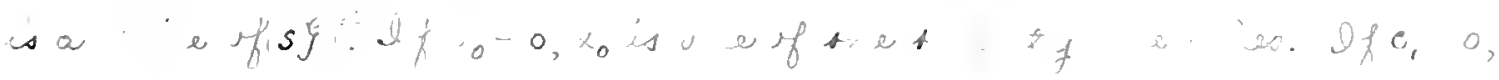

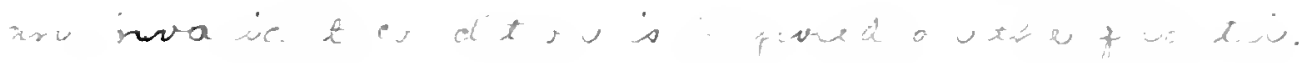



19

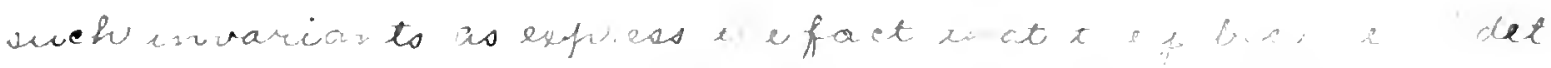

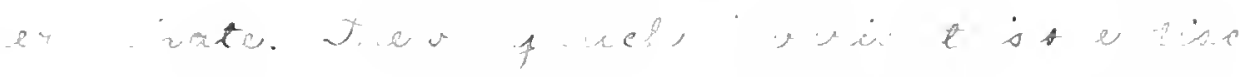
t

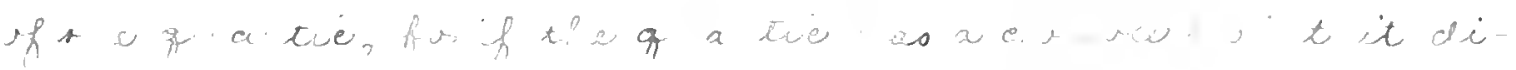

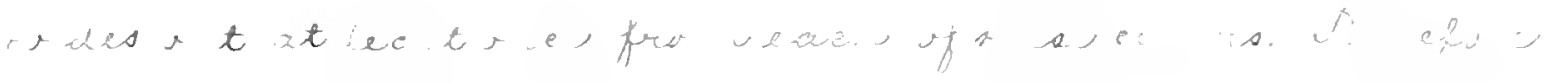

$$
\begin{aligned}
& A^{558} x^{43}=A^{2} x^{2,16} \cdot A^{-4} x^{2+4},^{2} \cdot A^{-0} x^{24} \cdot A^{27} \\
& \therefore 558=15 i+108+270 . \\
& 1, i+27 \cdots+3 c \text {. }
\end{aligned}
$$

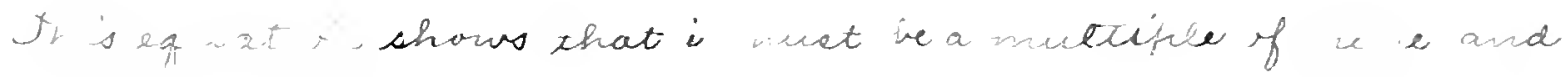

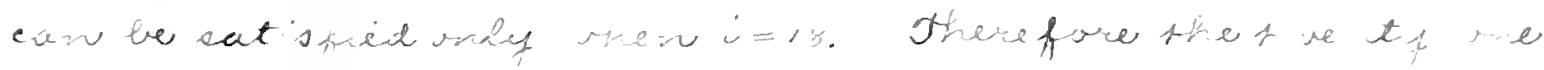

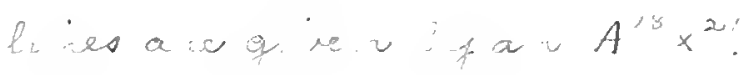

\$7. The Invariant of lakewern Deguee.

The clegree of the forns giving the tiventy-ores lines be iq

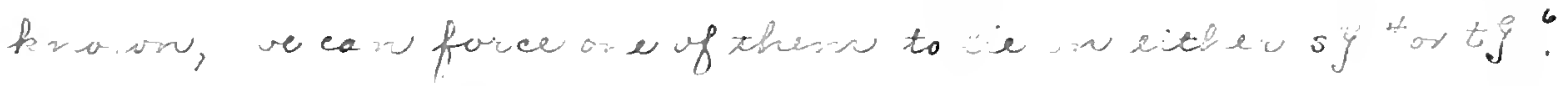

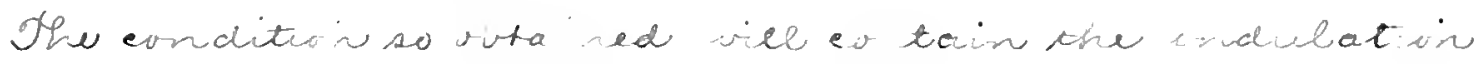

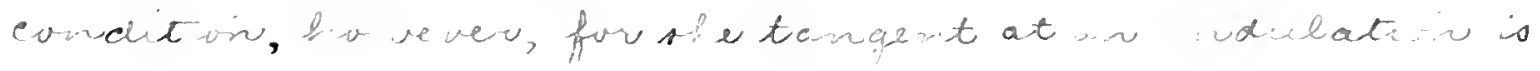

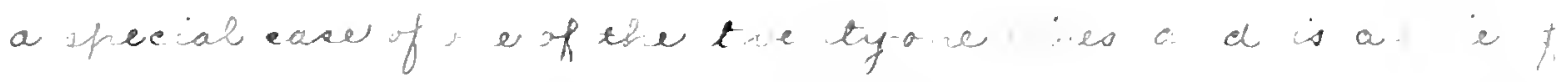

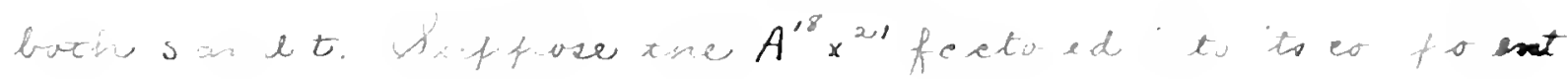


18

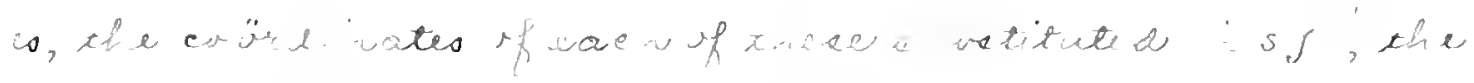

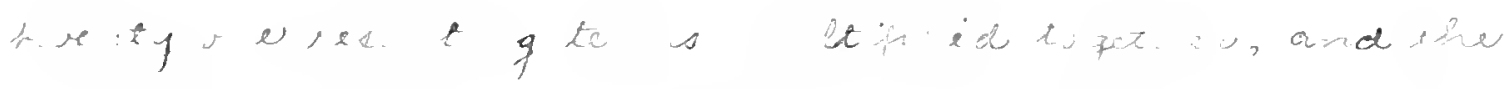

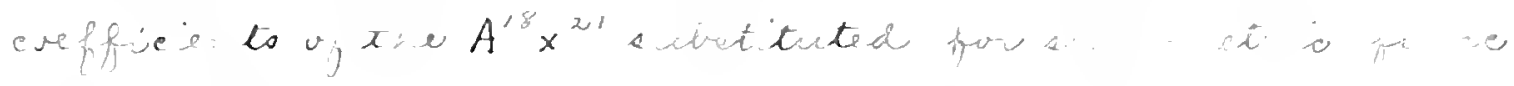

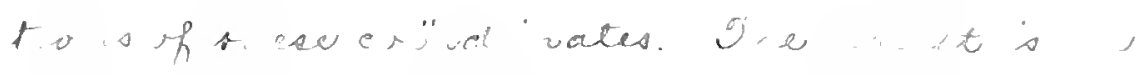

$$
A^{15 \cdot 4+2 \cdot 21}=A^{\prime \prime 4}=A^{60} \cdot A^{54} \text {. }
$$

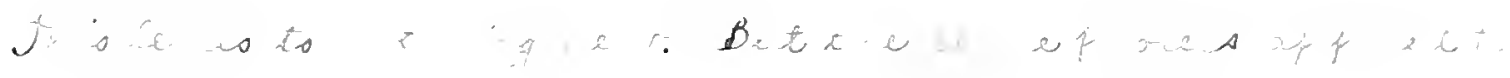

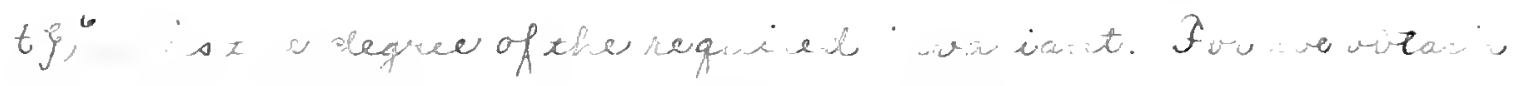

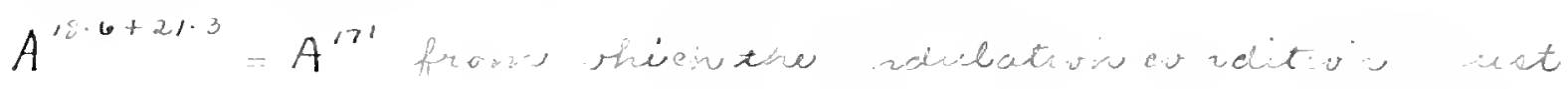

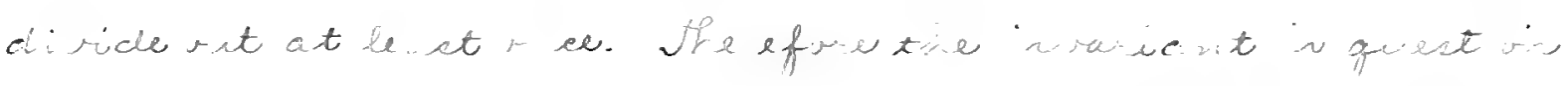

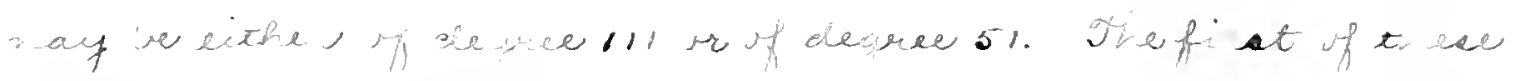

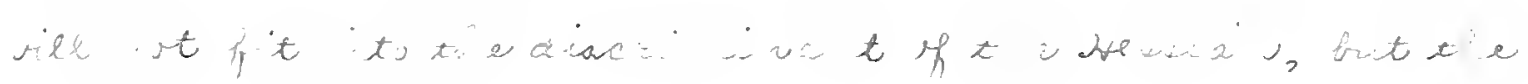
zeco d o d eldio

$$
A^{225}=A^{27} \cdot\left(A^{48}\right)^{2} \cdot\left(A^{51}\right)^{2},
$$

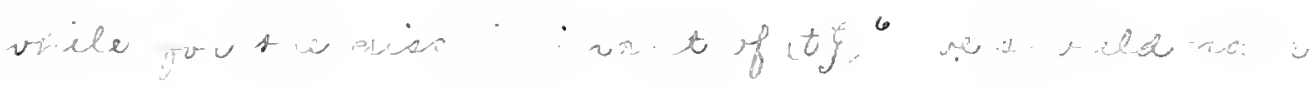

$$
\left.A^{225}=A^{21},^{2} \cdot A^{60}\right)^{2} \cdot A^{51} \text {. }
$$

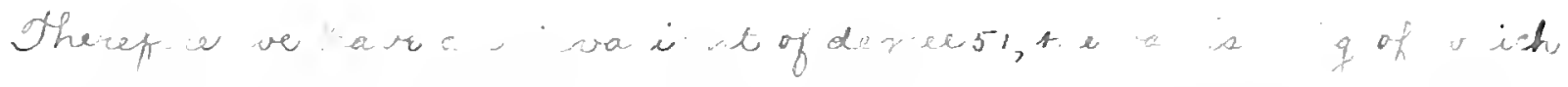

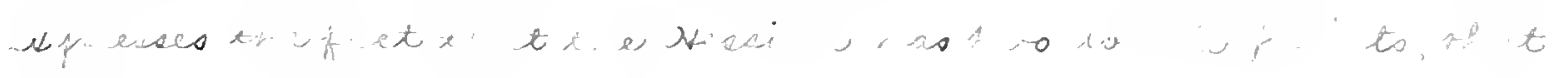

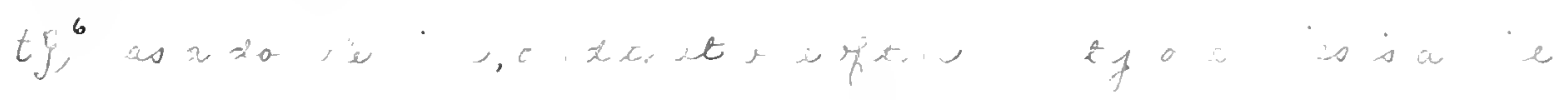
otit," A further maning will be seen later. 

$\$ 8$ berta, + yo is $x$

The degree of the form giving the twenty wee is $t$ iq

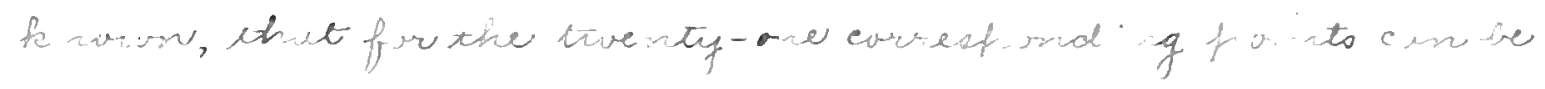

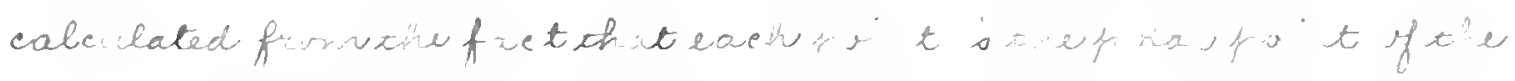

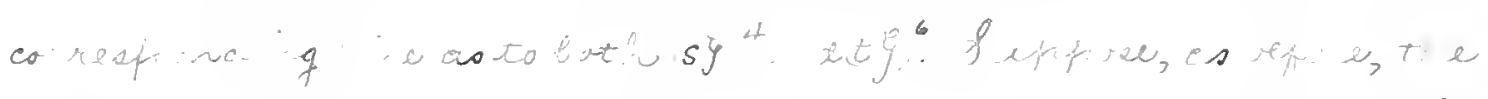

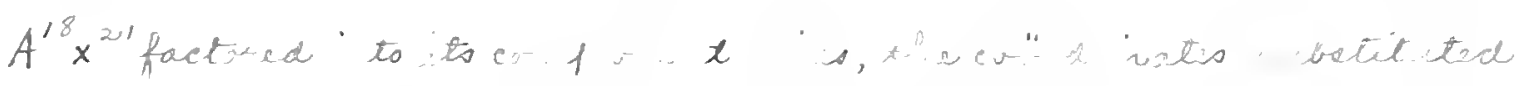

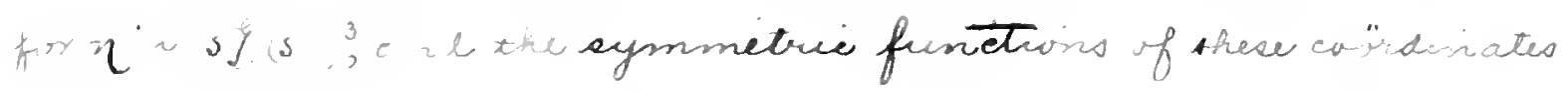

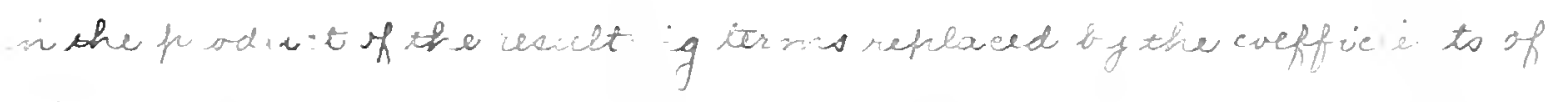

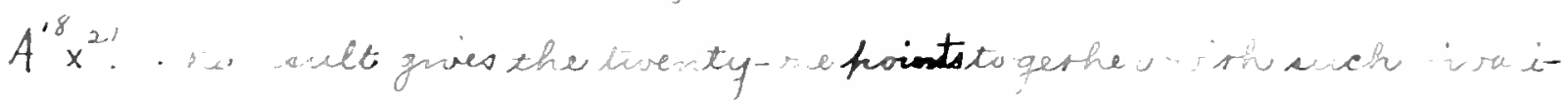

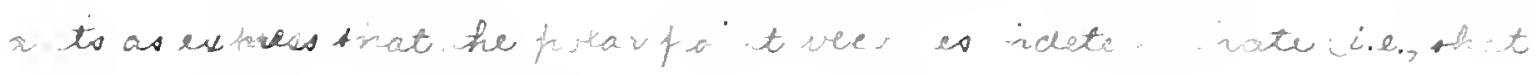

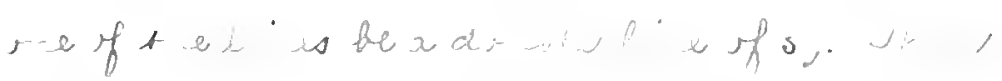

$$
A^{18 \cdot 3+2 \cdot 21} g^{21}=A^{544} \cdot A^{42} g^{21} .
$$

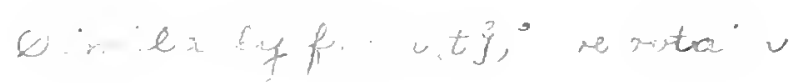

$$
A^{18 \cdot 5+3 \cdot 21} g^{21}=A^{60} \cdot A^{51} \cdot A^{42} j^{2} .
$$

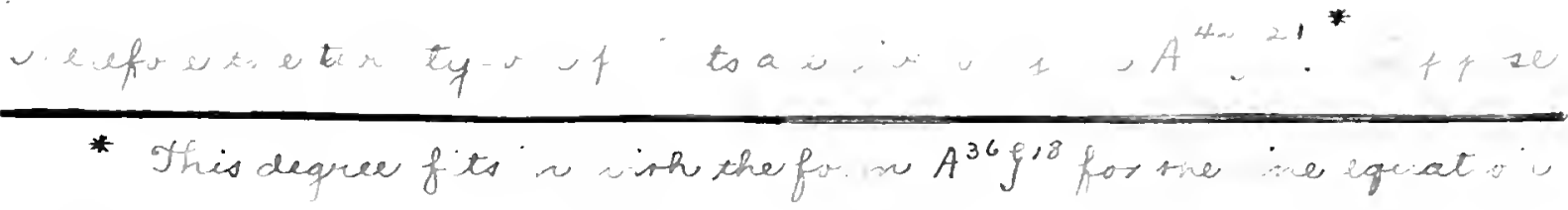

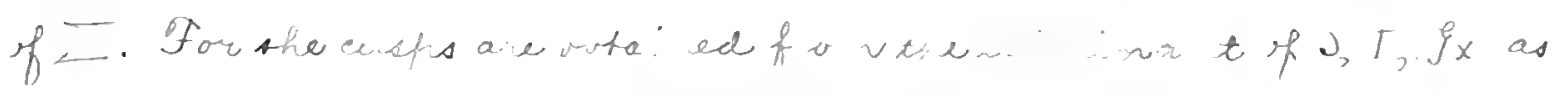

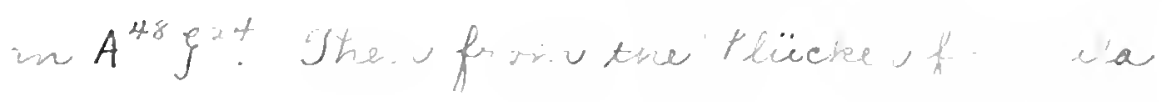

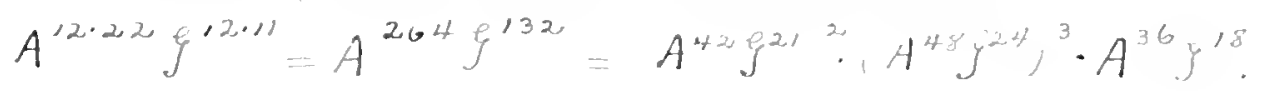


$2 \cdot 0$

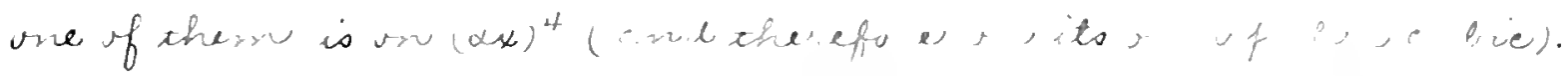

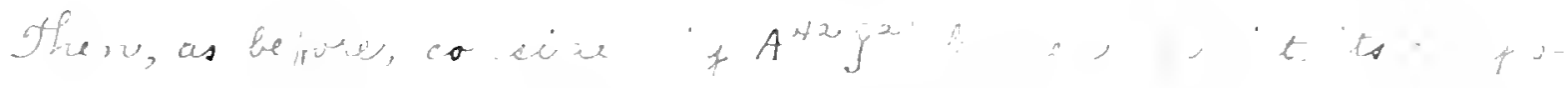

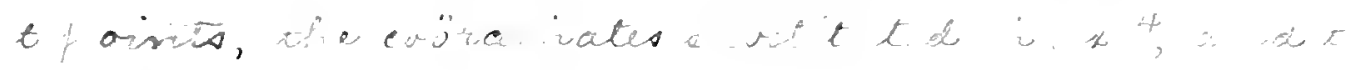

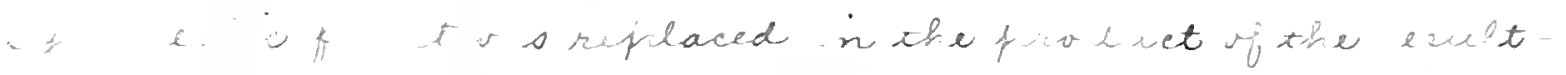

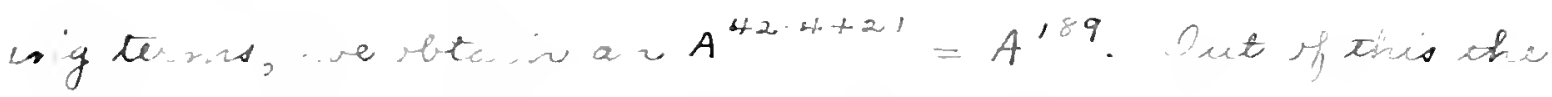

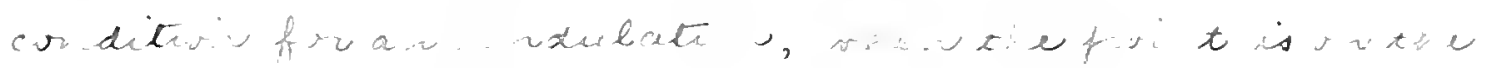

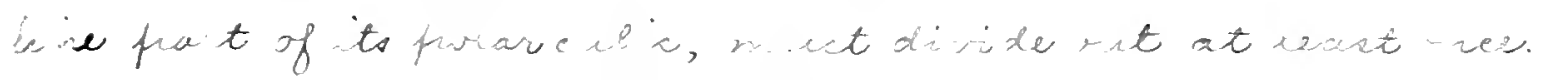

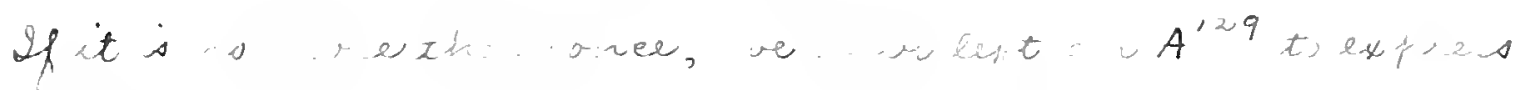

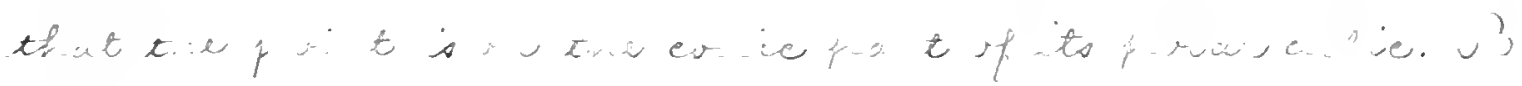

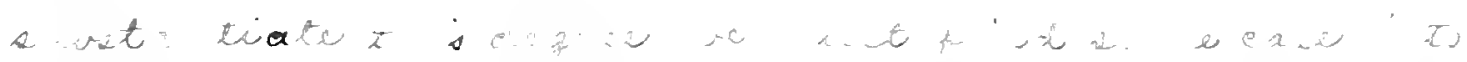

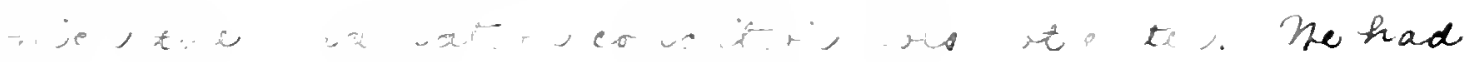
befow that the fwit is $\left(1,0,0\right.$ ) and ito cordesturnduig lini $x_{0}$ if

$$
b_{0}=i n=n=c_{0}=c .
$$

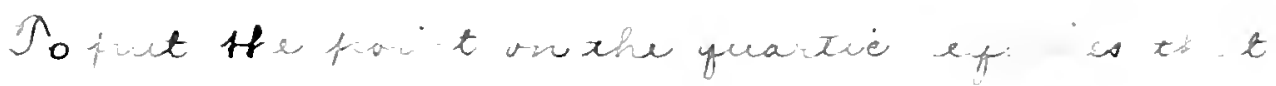

$$
x=0 .
$$

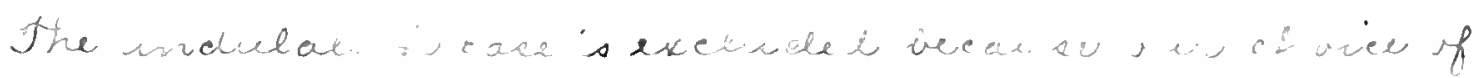

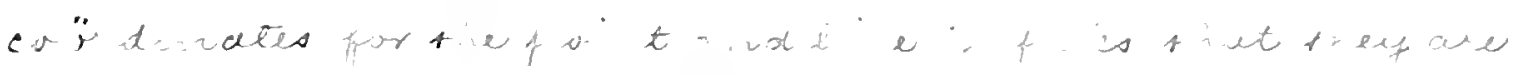

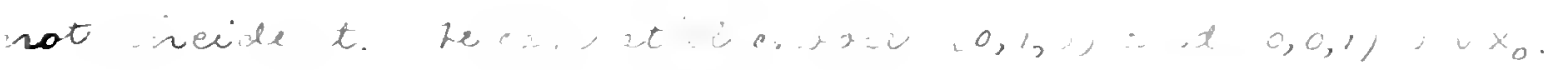



2,

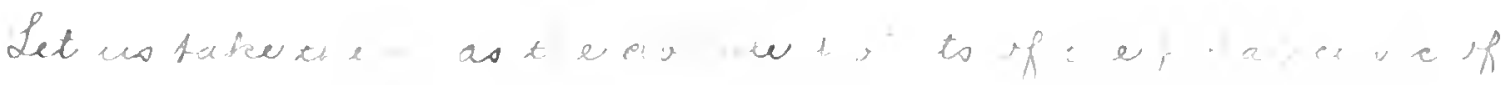
$10,2, \infty x: t$

$$
q=-c .
$$

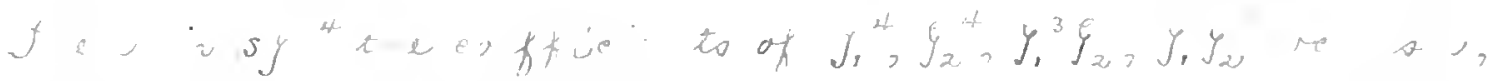

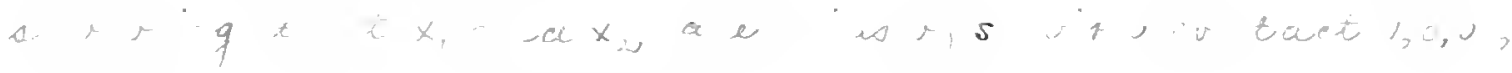

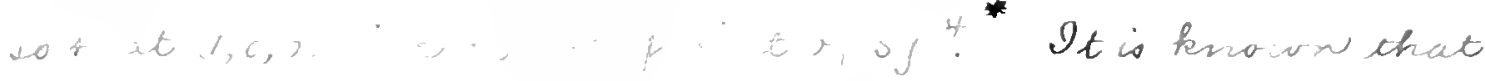

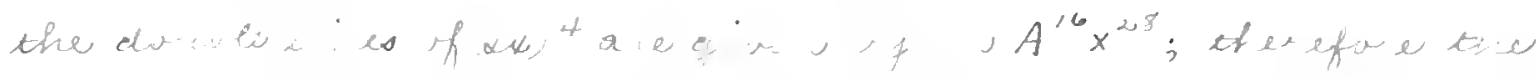

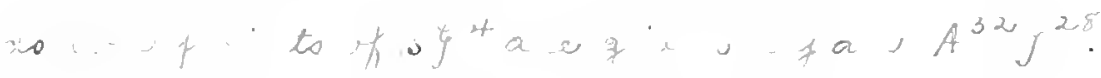

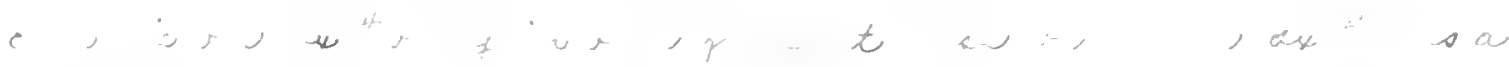

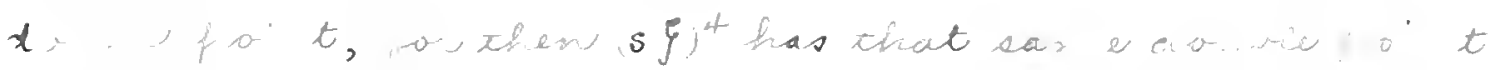
itu the se. to rap, to. Then by the method of cv sedering the $A^{32} g^{28}$ factored into its compo et t fast, we ave

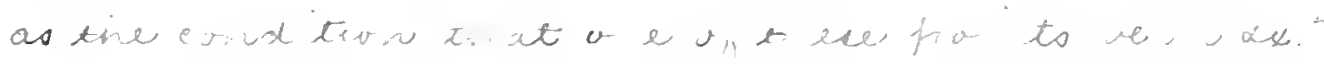

$$
A^{32 \cdot 4+25}=A^{156}=A^{129} \cdot A^{2 / 3} \text {. }
$$

This establishes the degree of the $A^{\prime 2}$ ?

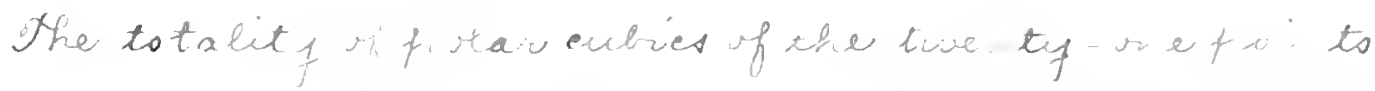

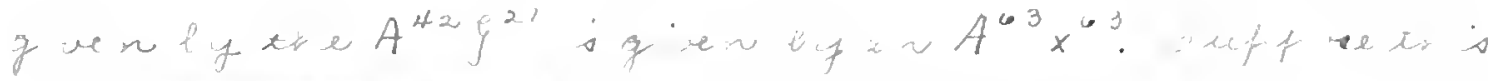

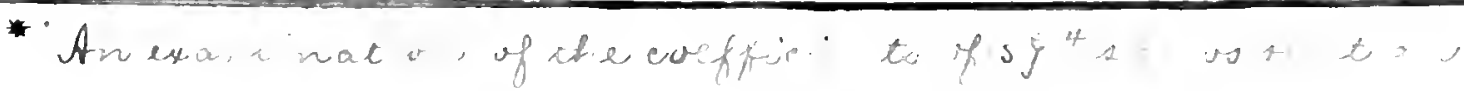

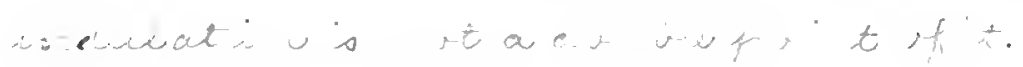




$$
\bullet
$$


2.2

factored into its component rubies $\left(\rho_{i} x\right)^{3}$, and then each of these cuties factored into its component line and conic $\left(y_{i} x\right) \cdot\left(\delta_{i} x\right)^{2}$. Then the condition that the line touch the conic is of second degree in both $\gamma_{i}$ and $S_{i}$, therefore of secone degree in $\beta_{i}$. Therefore the invariant expressing the condition that there be a polar cubic made up of a conic and a live touching it is an $A^{6 \cdot 2}=A^{126}$. This result can be substantiated in ocher rays. If, as usual, we take the lIne as $x_{0}$ and the corresponding faint as $(1,0,0)$, so th at

$$
b_{0}=n=n=e_{0}=0 \text {, }
$$

then the eric with towed $x_{0}$ at $(0,1,0)$ if

$$
h=l=0 \text {. }
$$

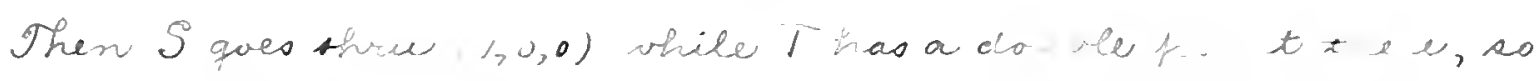

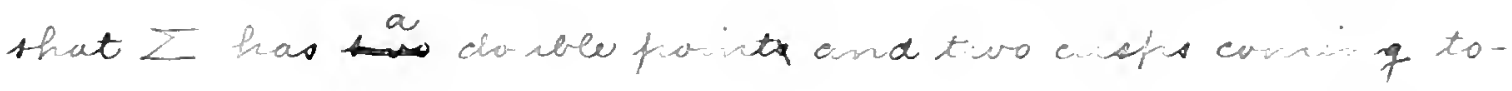
getter and acquires a turtle prim t, since $x_{0}{ }^{9}$ is the nighest power of $x_{0}$ recurring in ito equation. S ire two of the intersections of $S$ ad Thane corner to peckers, the $A^{\prime 26}$. we th

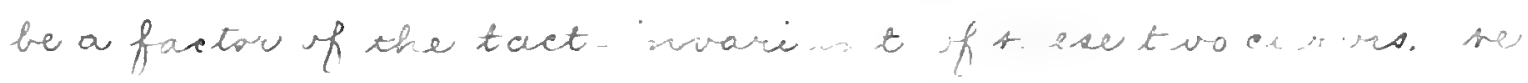
haves en $(p, 8,10)$ that in case of to e ia. is iq if titi 

3,3

the $A^{48}$ or the $A^{51}$ that $S$ and $T^{-}$touch at two distinct points, sind these three cases seem to be the only ones in which two intersections of cheer curves come to aether. Their tact -invariant is found frow the formula given by folmon to be of degree H. $(6+8-3)+6 \cdot 4(4+12-3)=57^{6}$. Then $A^{576}=\left(A^{48}\right)^{2} \cdot\left(A^{51}\right)^{2} \cdot\left(A^{126}\right)^{3}$.

Hes inspection of their coefficients shows that both it ind $S$ touch $x_{0}$ at $(0,1,0)$. As will be seen later, two intersections of $S$ and $H$ can came tagethein only when two of $S$ and $T d o$. Then the $A^{\prime \prime}$ vanishes, $H$ has two double fruits on $S$; when che $A^{48}$ vanishes, H has a deere front which is ales a double point of $\boldsymbol{S}$. The tact-imvariant of $S$ and $H$ is of degree $4 \cdot 6(6+8-3)+3 \cdot 4(4+12-3)=420$.

$$
A^{420}=\left(A^{48}\right)^{4} \cdot\left(A^{51}\right)^{2} \cdot A^{126} .
$$

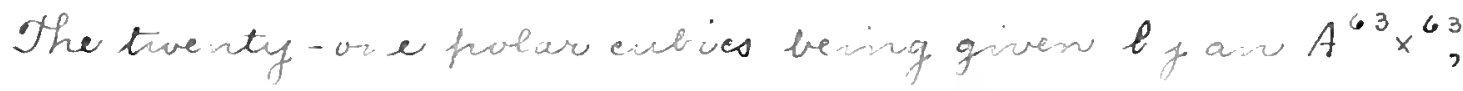

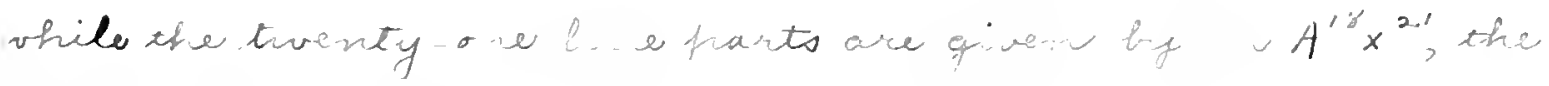

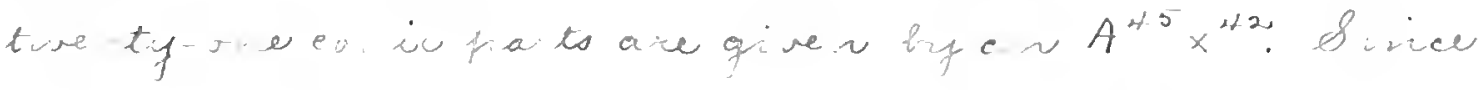

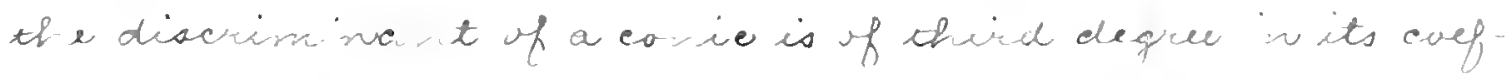

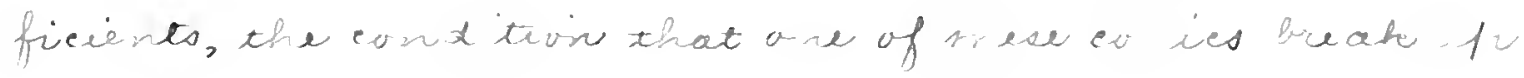



21

is an $A^{45}$. But the the f ware $b$ ic ev sisto of ifpee es,

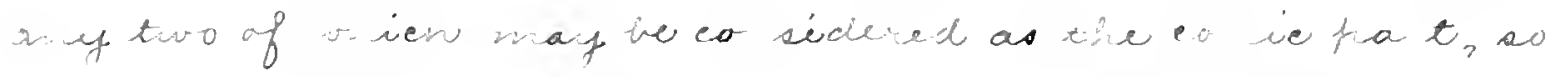

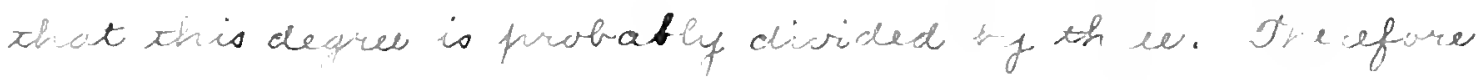

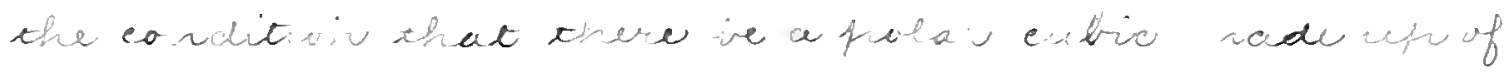
chree bires is an $A^{45}$. Then three of the dowere porits of

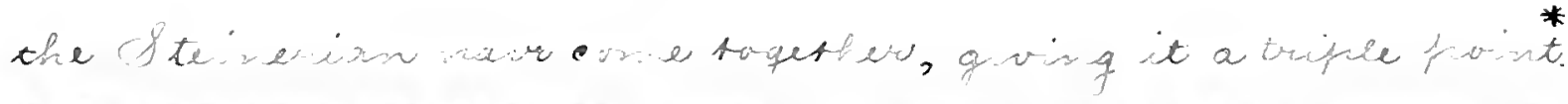

* Profecsur do reley has poirted o ct to w chet the dequess of

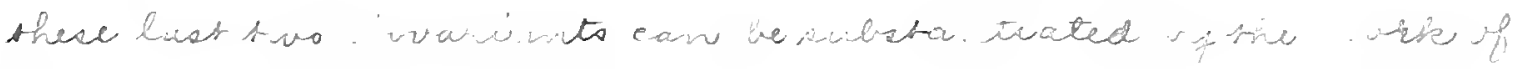

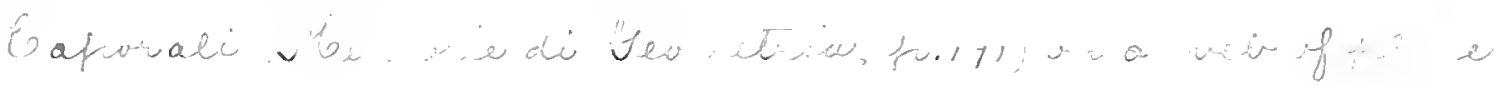

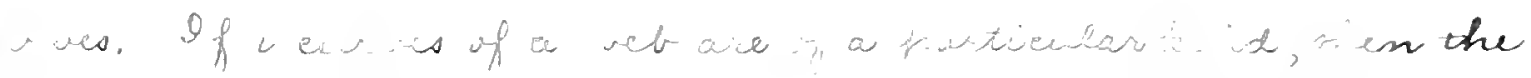

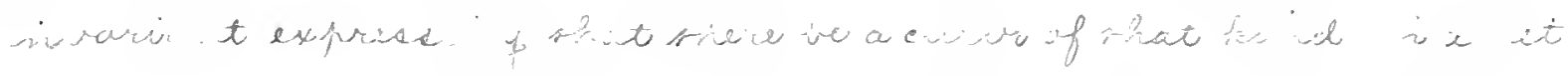

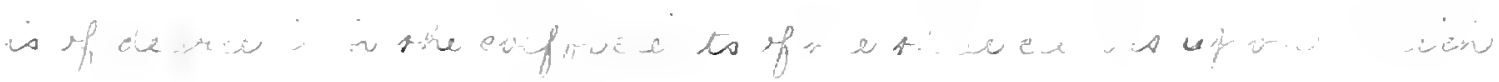

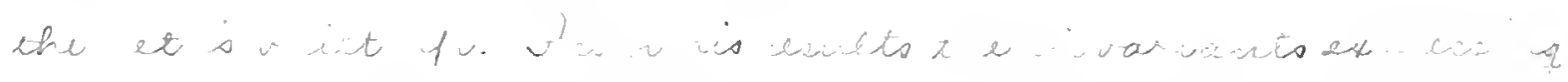

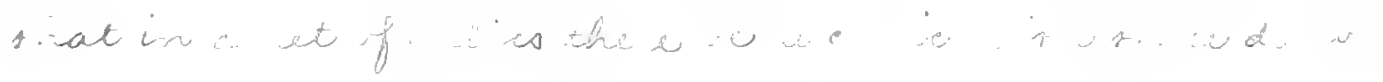

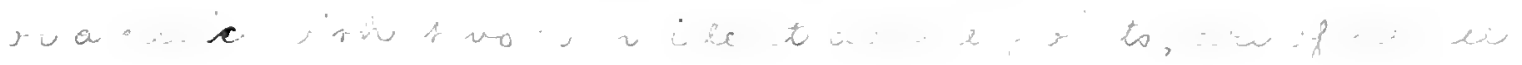

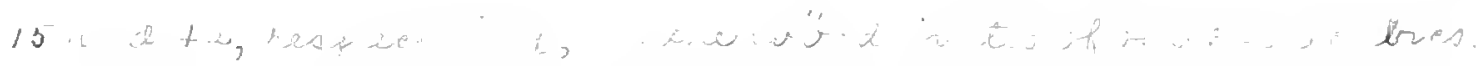

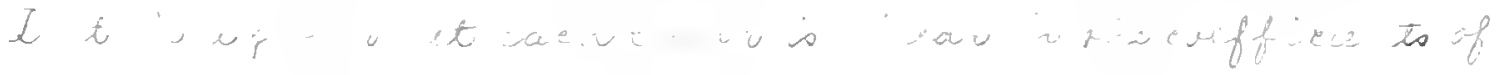

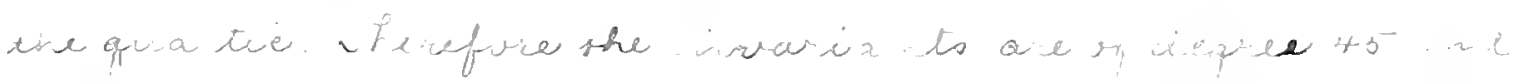
126 in the eveftice to of the grata lie. 
2,3

$\S q$ The Elerrinant of $, i, t$

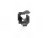

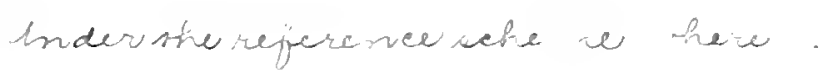

$$
b_{2}=f=n=0,
$$

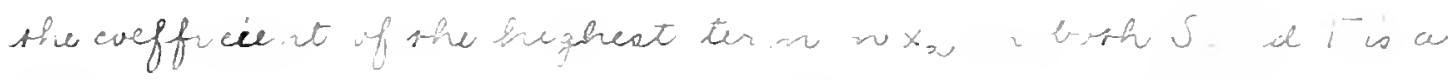

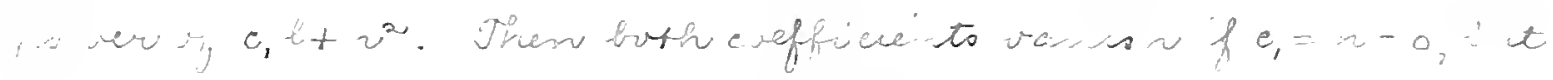

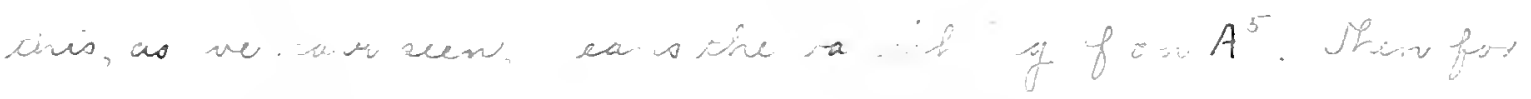

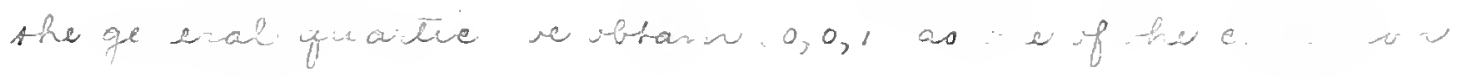

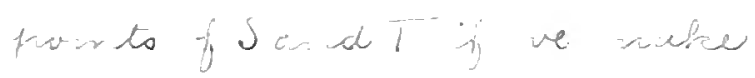

$$
i=n=0 .
$$

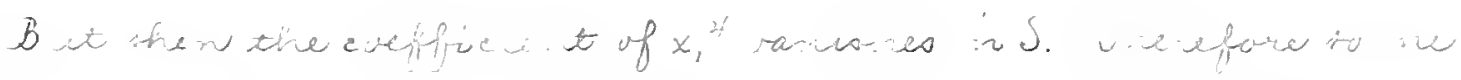

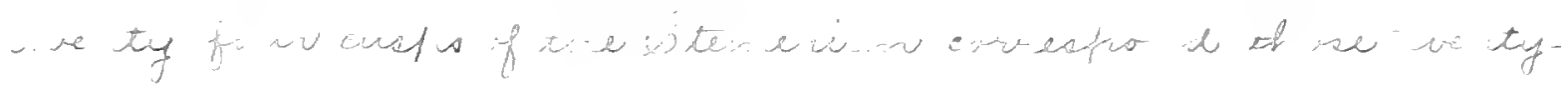

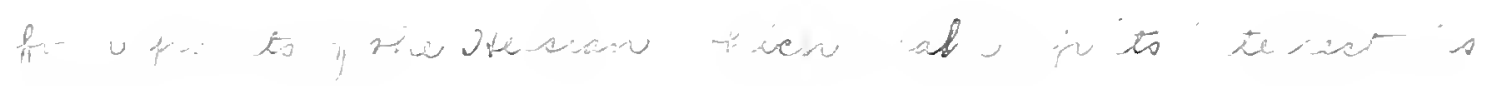
ito.

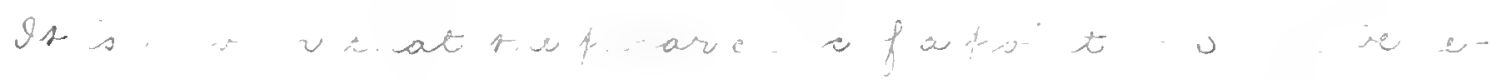

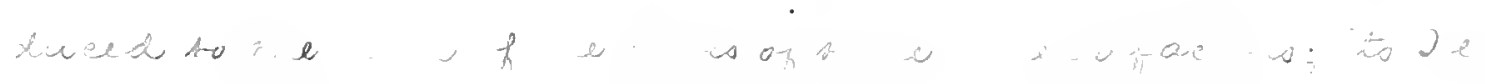

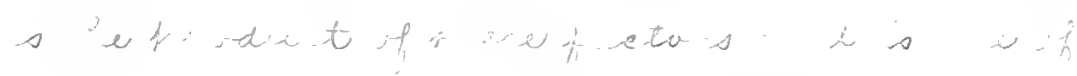

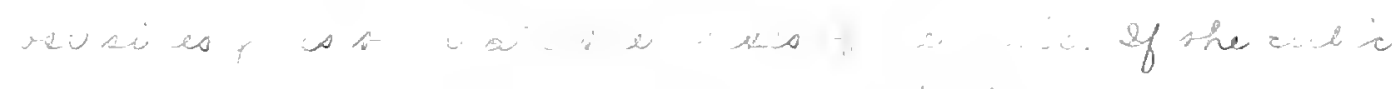
hen pees

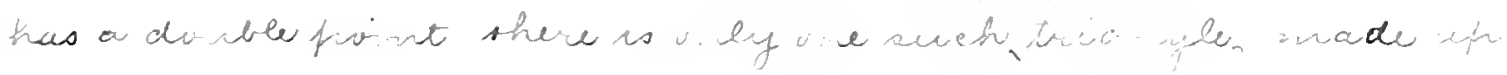



20

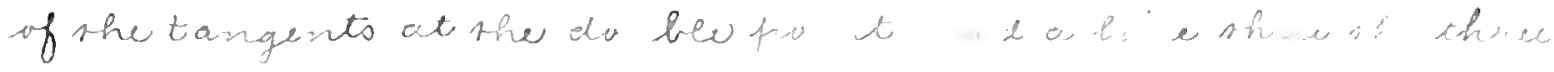

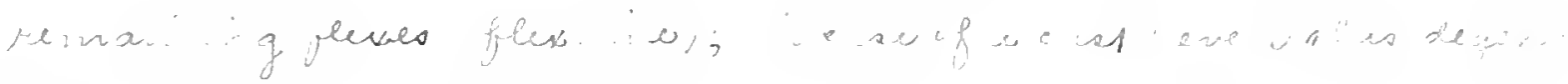

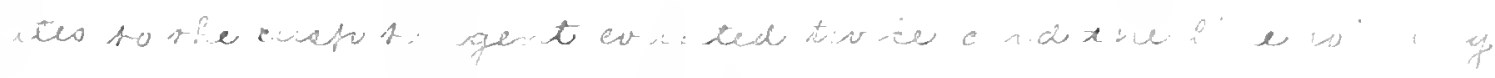

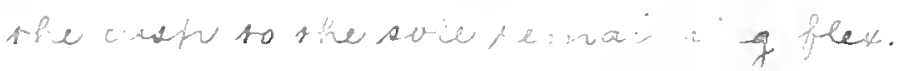

Dere she fulare che of $0,0,1)$ wo

$$
a_{2} x_{0}{ }^{3}+3 g x_{0}^{2} x_{2}+3 e_{0} x_{0} x_{2}^{2}+3 c_{1} x_{1} x_{2}^{2}+c x_{2,3}^{3}
$$

and its beteidion is

$$
c_{1}^{2} x_{2}^{2}\left(c_{2} x_{0}+g x_{2}\right)
$$

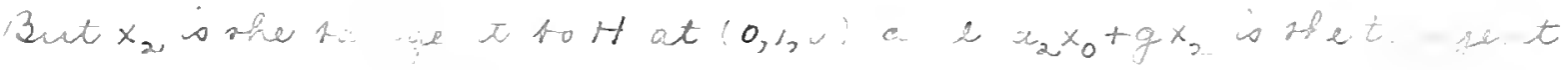

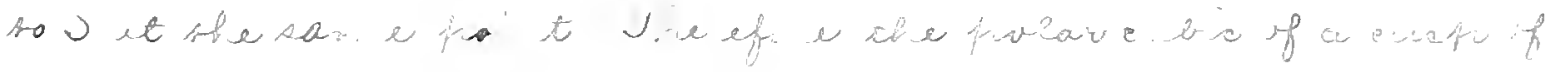

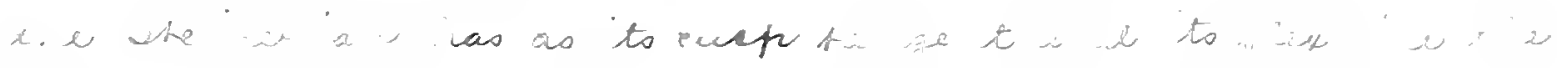

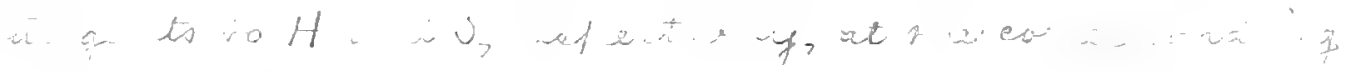

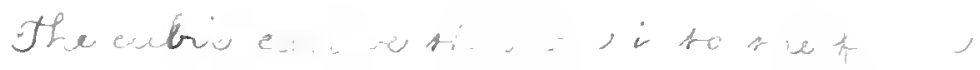

$$
\frac{1}{a_{2}^{2}}\left[\left(a_{2} x_{0}+g x_{2}\right)^{3}+x_{2}^{2}\left\{3 a_{2}\left(a_{2} c_{0}-g^{2}\right) x_{0}+3 a_{2}^{2} c_{1} x_{1}+\left(c a_{2}^{2}-g^{3}\right) x_{2}\right\}\right]
$$

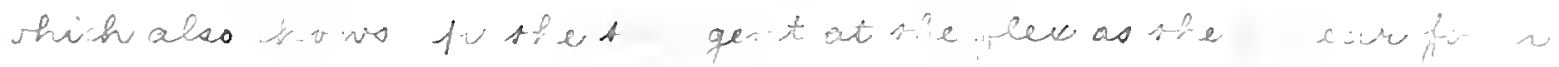
in buefreto

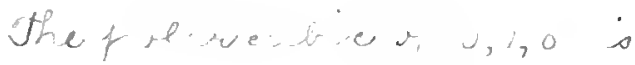

$$
x_{1} x_{0}^{3}+3: k_{0}^{2} x_{1}+3 b_{0} x_{0} x_{2}^{2}+b_{1}+c_{1} x_{2},
$$

ar to syesain is 
27

$$
c_{1} x_{2}\left[x_{0}^{2}\left(a_{1} b_{0}-h^{2}\right)+x_{0} x_{1}\left(b a_{1}-b_{0} h\right)+x_{1}^{2}\left(b h b_{0}^{2}\right)\right] .
$$

Then she tangent to that $(0,1,0)$ pasacs thew thice fleses of

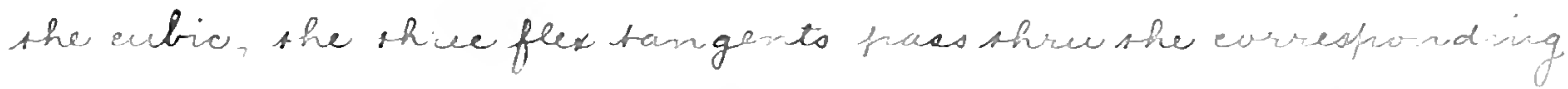
Steinerian twont and she b inory descian vithese tien tom

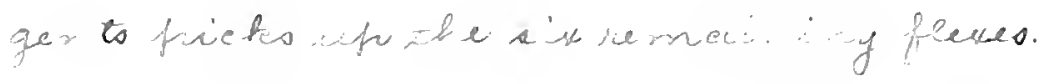

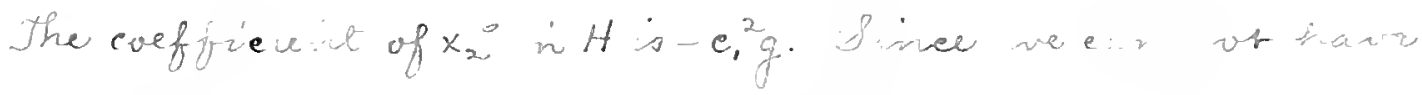

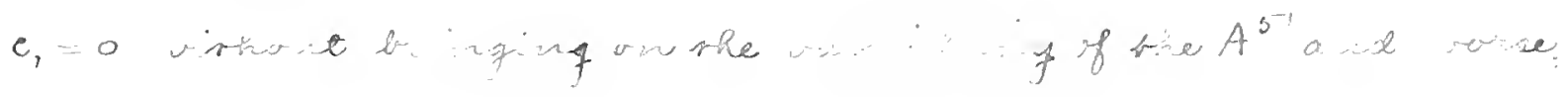
let wo shec

$$
q=0 \text {. }
$$

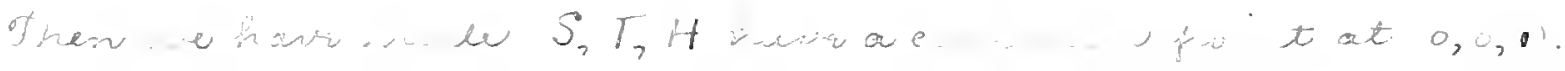

The polar ecico of $, 3,1$, thecomes

$$
a_{2} x_{0}^{3}+x_{2}^{2}\left(3 e_{0} x_{0}+3 c_{1} x_{1}+e_{2}\right) .
$$

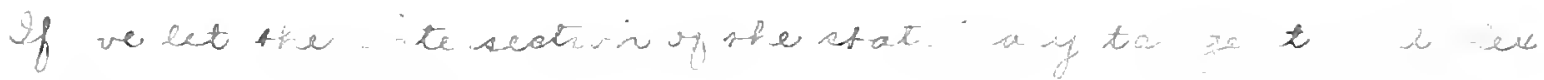
$l^{2}, 2,2,0,0,8,2: 2$

$$
c_{0}=0
$$

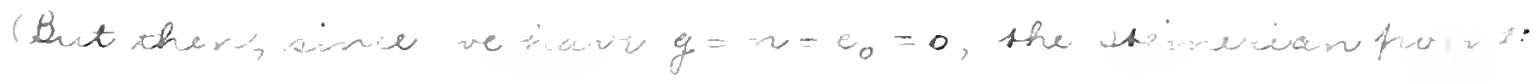

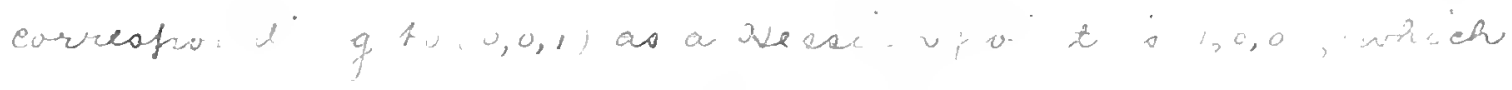

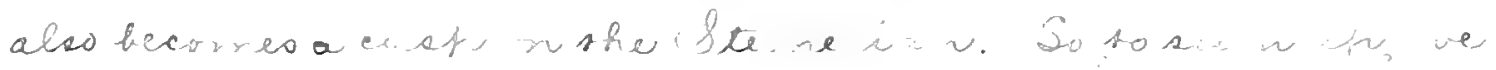

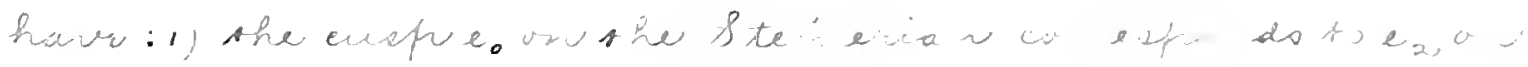



28

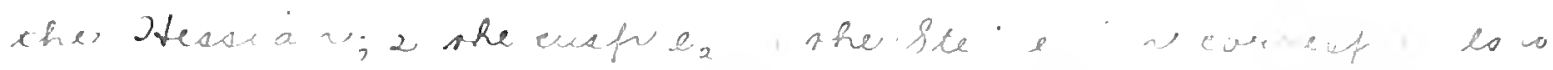

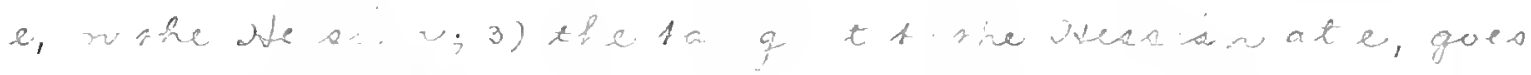

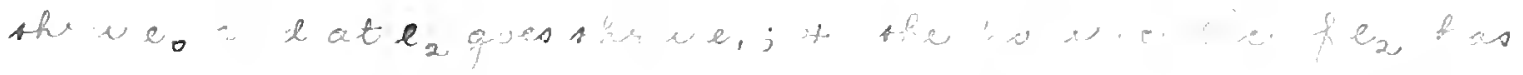

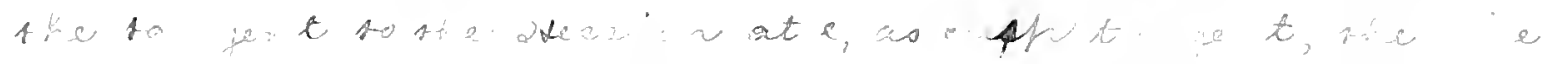

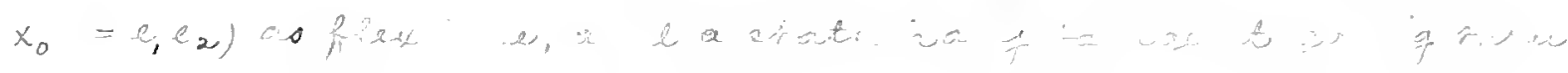

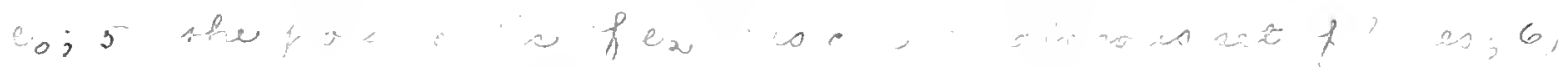

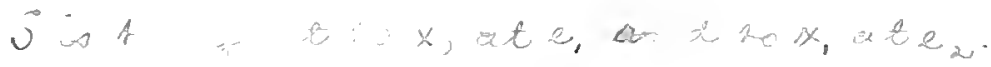

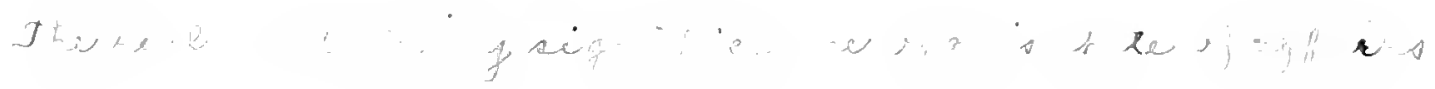

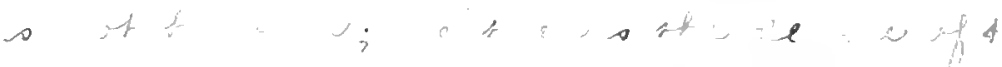

$\therefore t$ t t $T_{2} H$ is, $A^{300}$.

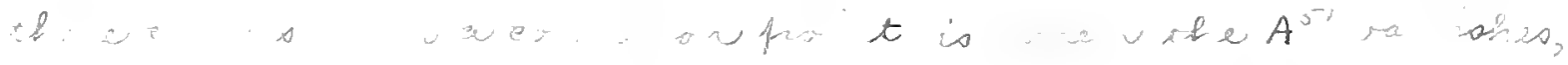

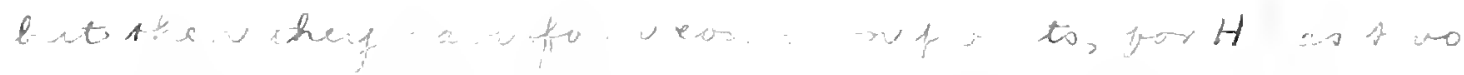

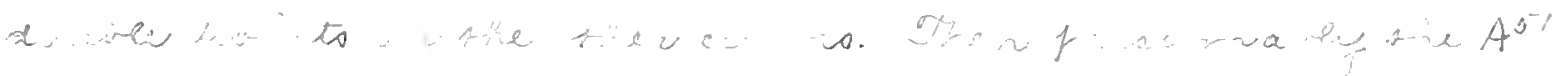

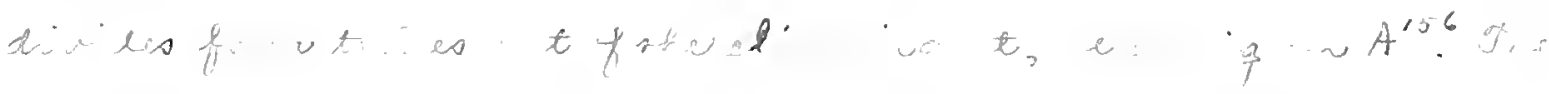

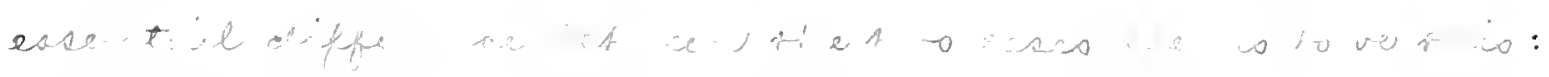

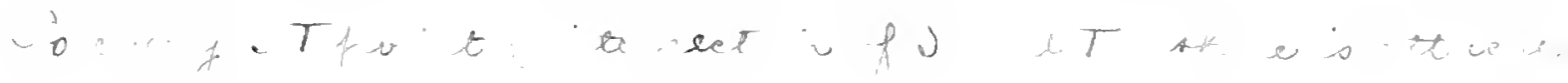

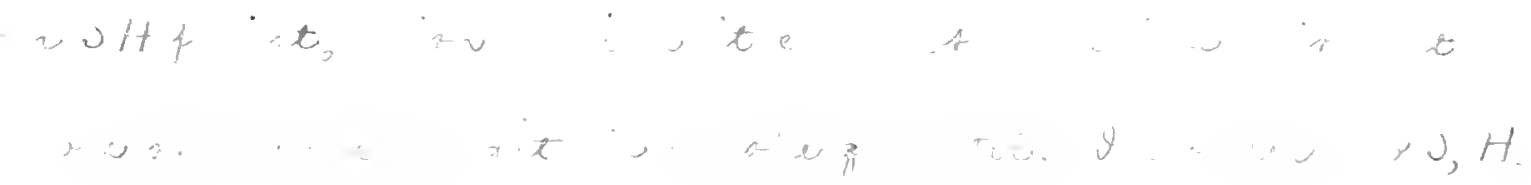


2.9

\$10. The Elininant of the Volar bubic, Bonie, and Live.

Supt ose we aste that the fwewrenbe, convie, and line of

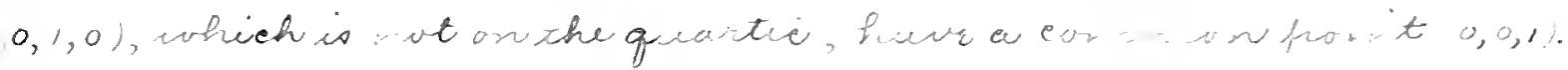

Thers

$$
b_{2}=f-c_{1}-0
$$

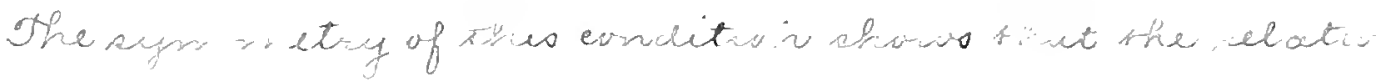
betweer, $(0,1,2)$ and $(1,0,1)$ is a watualo $e$. Heo, $x_{0}$ is a te

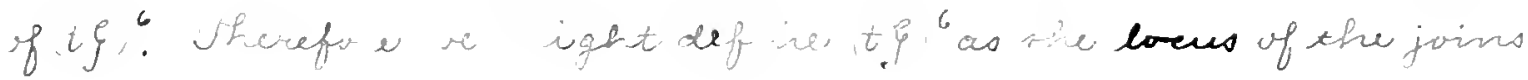
of pairs of pwints so related thot the fivlar encurs ffecech pass threw the other, the paur of fari to being the eas on w

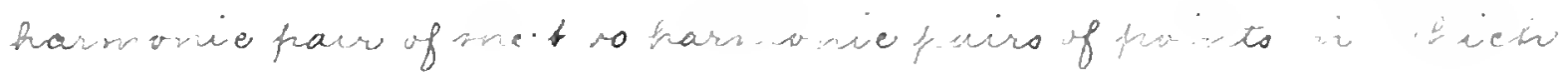
their join cats tine quantie.

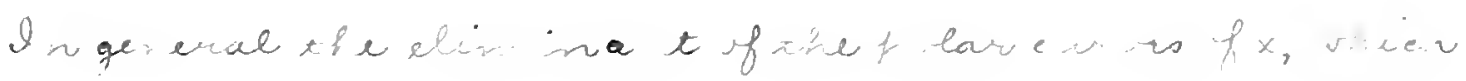



30

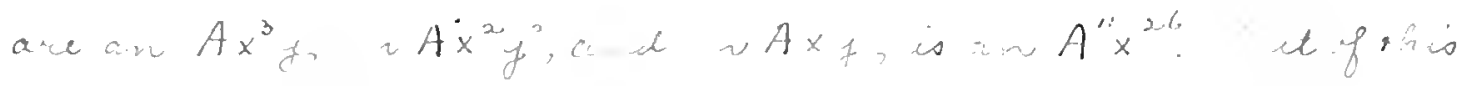

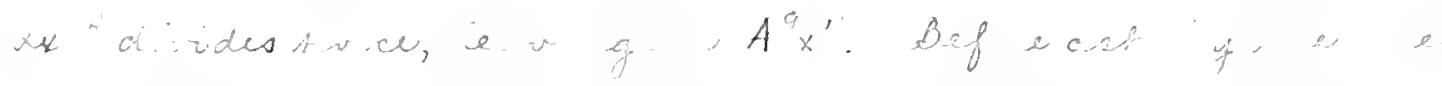

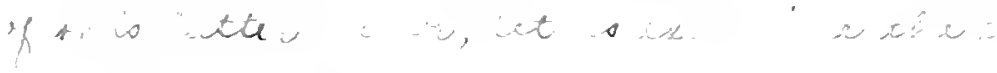

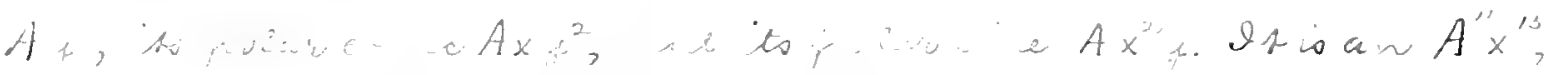

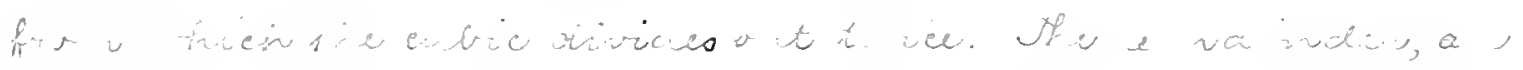

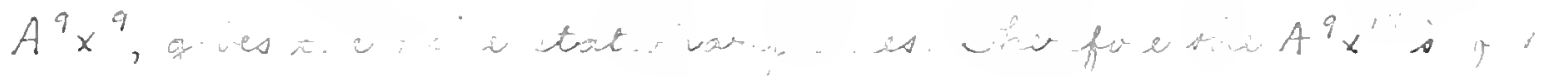

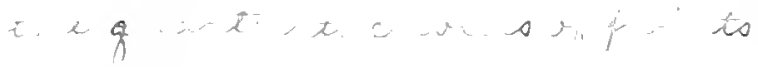

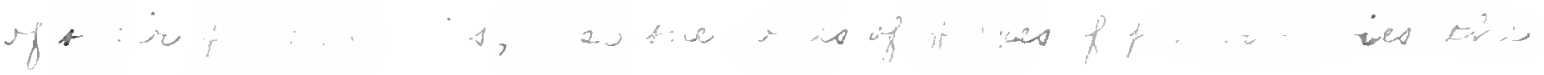

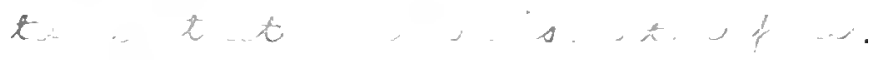

isalmory ge res as the equation of the ine statioivary Lis es of checule

$$
3>\iota^{2} H-H^{3}-l \theta \text {, }
$$

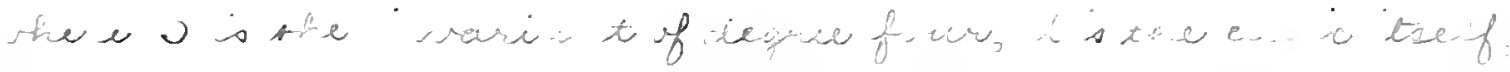

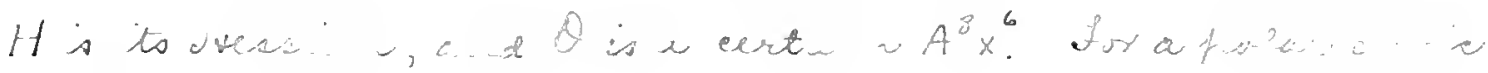

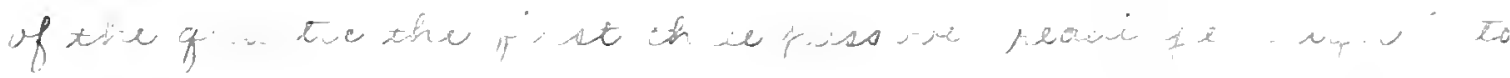

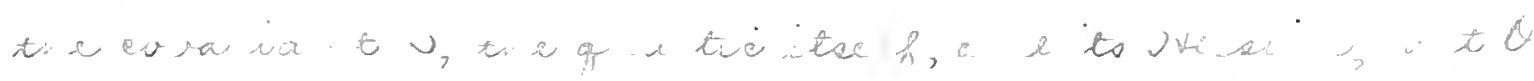
is of se exsiaf bo ster L2. So we vill substitute for it a nocher $A^{8} x^{6}$, expescible in lerns of $S, l, H$, and $\theta$, whech gives the locus of points whose polar ev. ies as to the cubie 

31

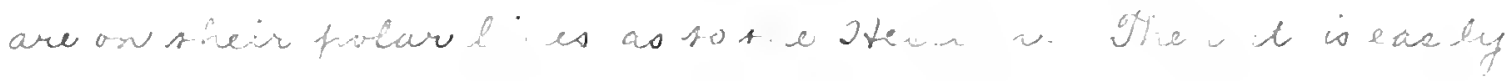
Qbarion a...e.

$$
A^{9} x^{\prime}=8 S L^{2} H H^{3}+9<\theta \text {, }
$$

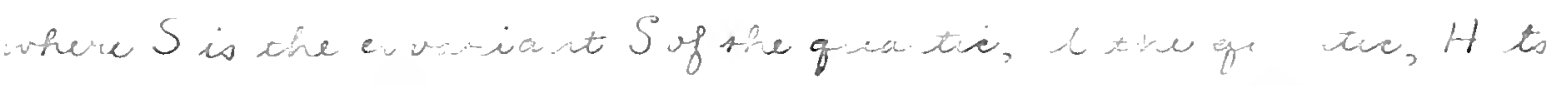

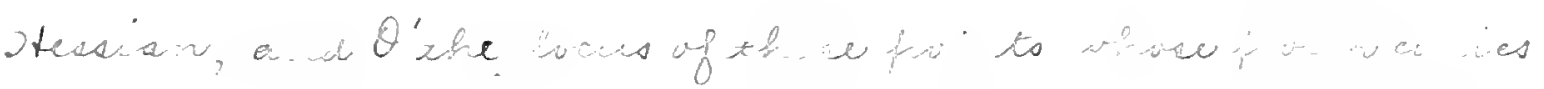

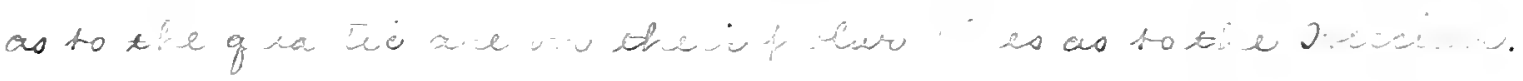

The fo of the $A^{9} x^{38}$ shows that all of to te wecto.

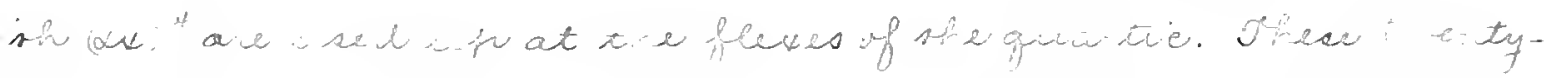

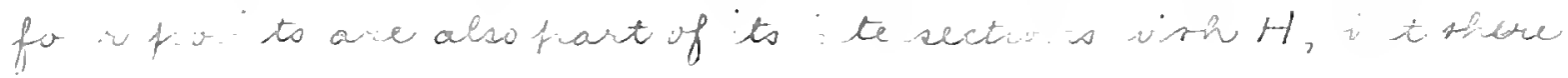

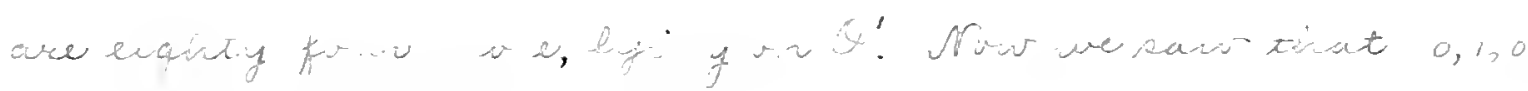
is a parit figtie $A^{9} x^{18}$ if

$$
\theta_{2}=f=e_{1}=j_{1}
$$

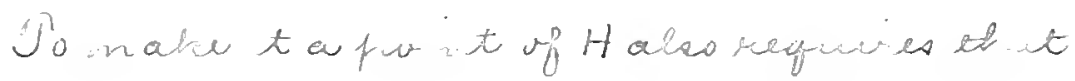

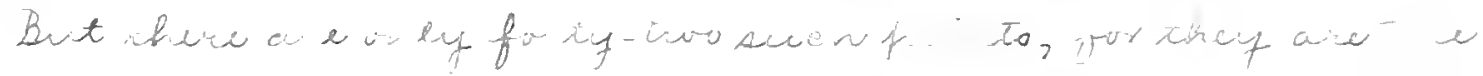

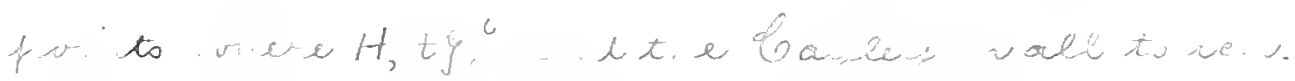

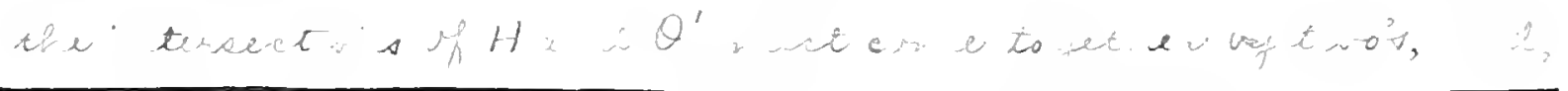

30 , sym etweally, 0,0, satpint of $H^{\prime} \ldots$

* Proc. Mat. Ac. Sci., vol. 3, p.titg (1q,7) 

32

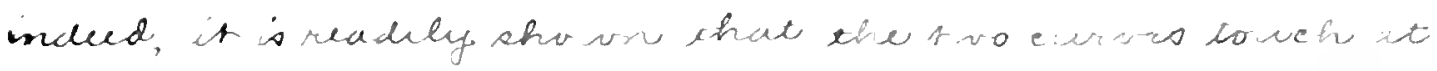
iblice no. for to

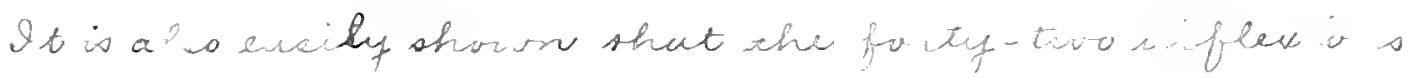

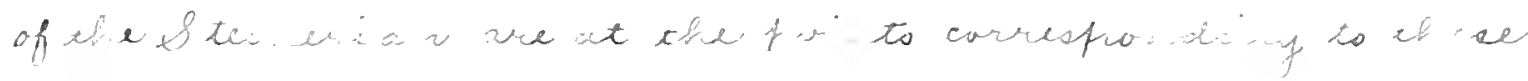
Jeacian fici: to.

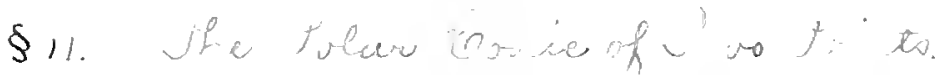

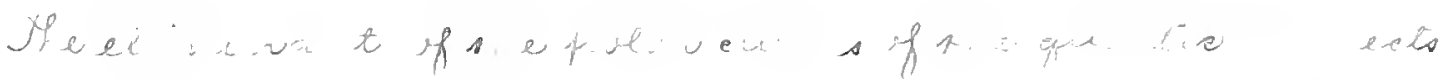

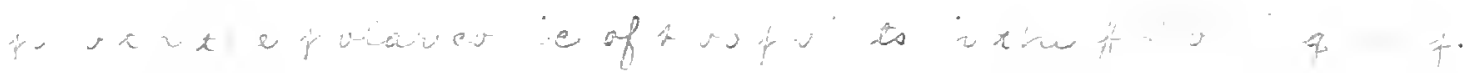

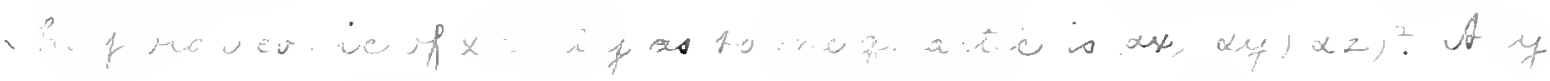

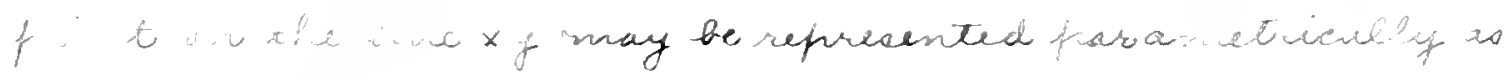

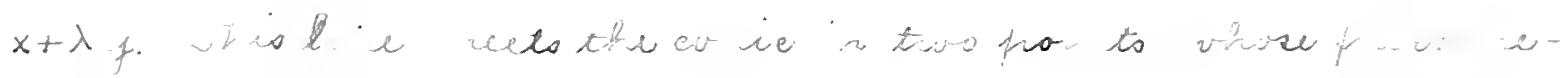
ters $\lambda$ ane gien if

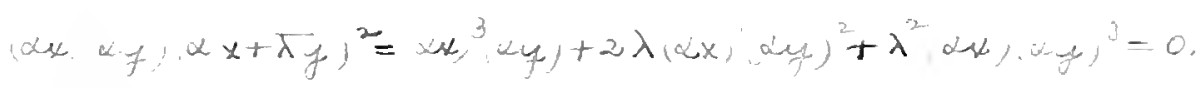

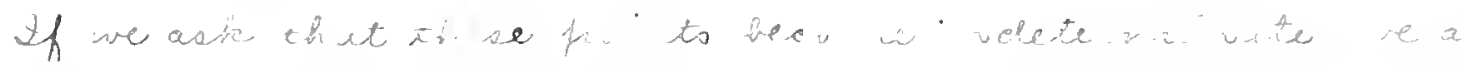

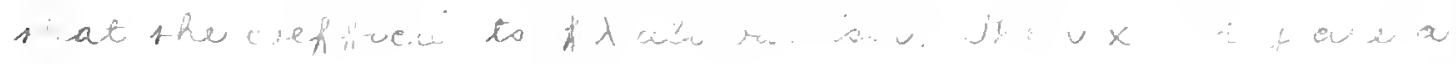

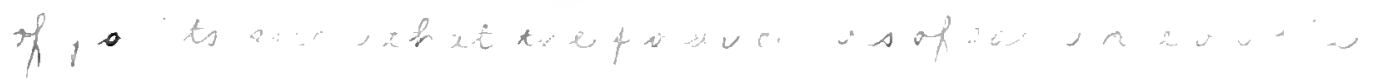

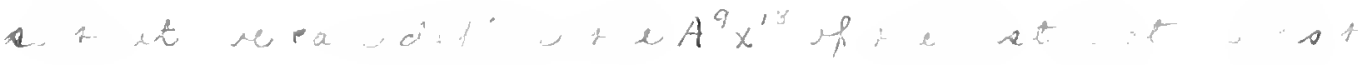

$\infty$ 



3

$$
v_{2}+0_{3}
$$

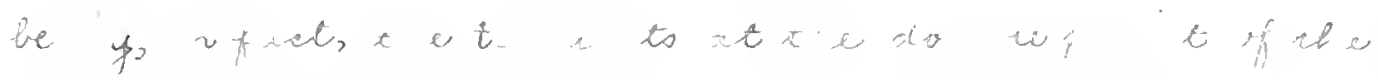

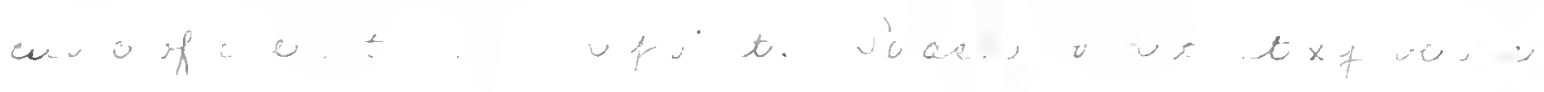

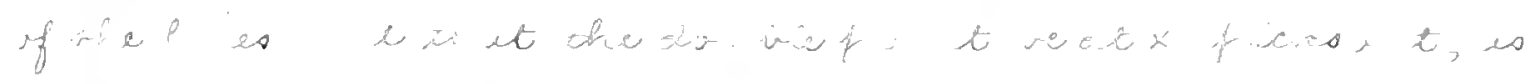

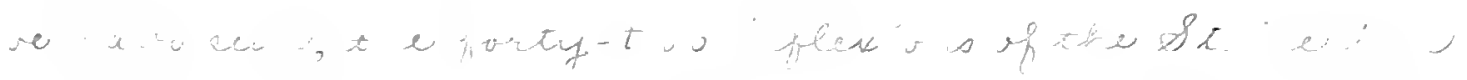

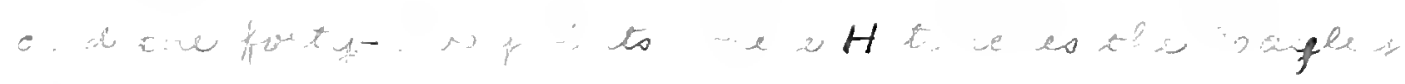
2. $\mathcal{L} \operatorname{ct} y^{6}$

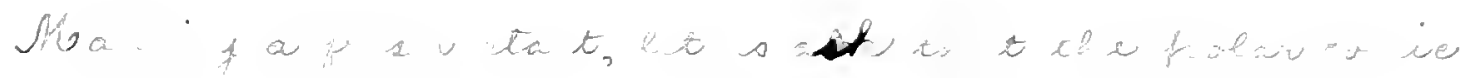

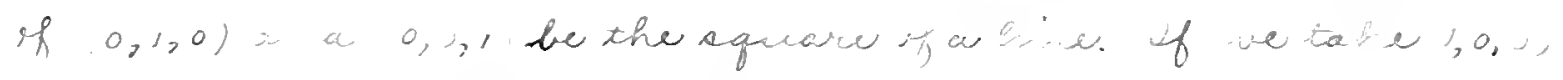
as a p...'tifis is the w

$$
\begin{array}{r}
l=, 2=-2, \\
b_{2} c_{1}-k^{2}=0 .
\end{array}
$$

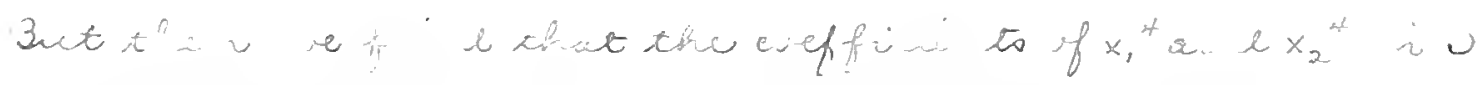

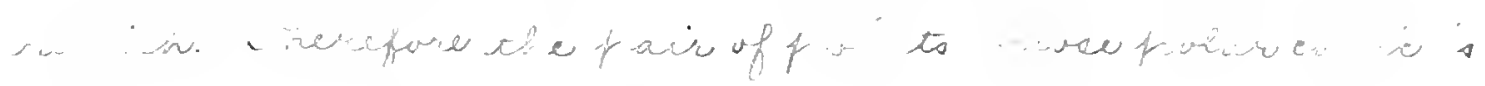

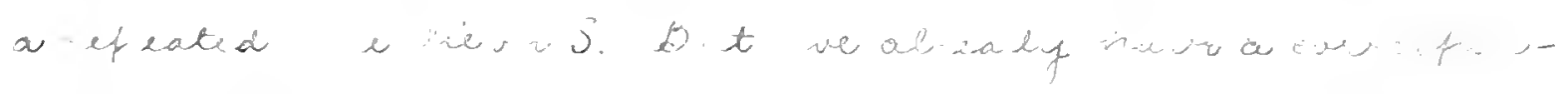

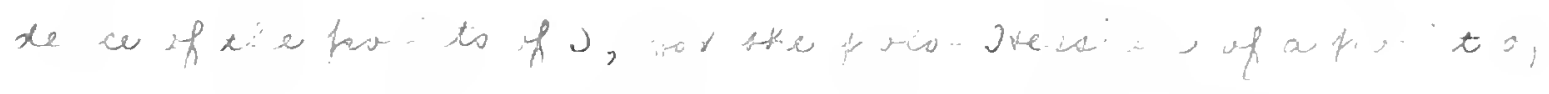

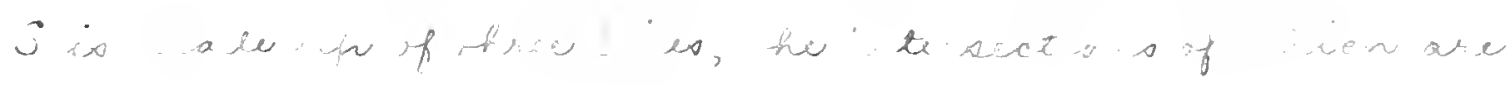

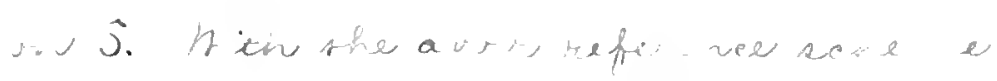

$$
\left.v_{4},-x_{1} v_{0}-v^{2}\right)\left(-x_{2} c_{0}+v^{2}\right)
$$



35

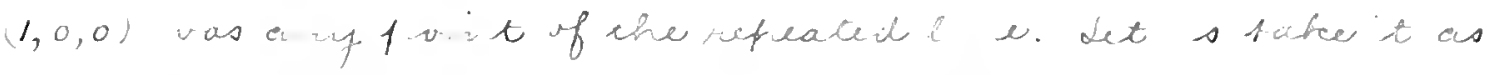

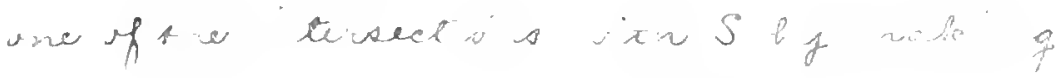
$a, b_{0} i^{2} 0$.

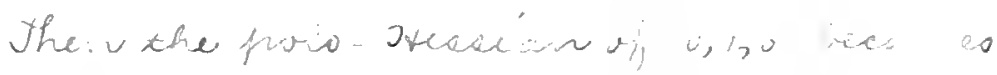

$$
\begin{aligned}
& x_{1} x_{0} x_{1}, b a_{1},-a_{1} v_{2}^{2}-v_{0}(h)+x_{2} x_{2}\left(a_{1} c_{1}-a_{1}, v_{2}\left(-v_{0} c_{1} v\right)\right. \\
& \quad+x_{1}^{2}\left(v, v-b_{0}^{2} f-v_{2}^{2} v\right)+x_{1} x_{2}\left(b c_{1} v-v_{0}^{2} c_{1}-v_{2}-v_{1}\right) .
\end{aligned}
$$

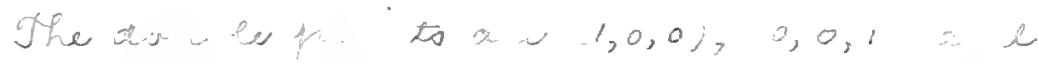

$$
f \equiv\left(v e_{1} v-v_{0}^{2} e_{1}-v_{2} f v,-b a_{1} e_{1}+a_{1} z_{2} f+b_{0} e_{1} v_{2}, a_{1} f-a_{1} v_{2}^{2}-b_{0} f h\right) .
$$

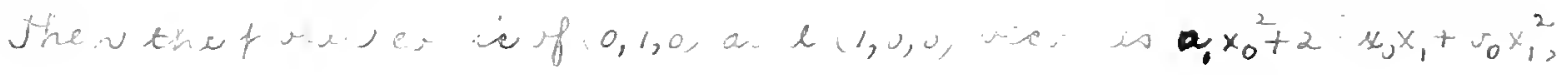

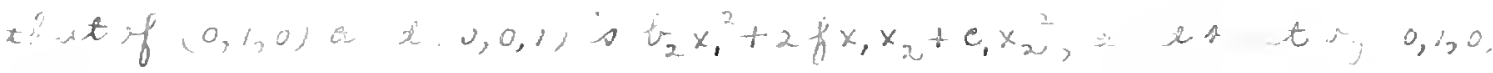

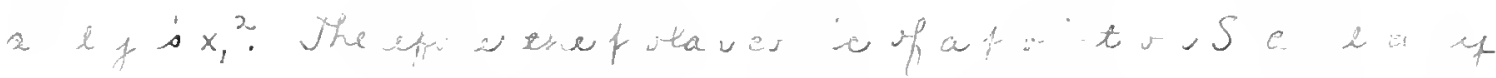

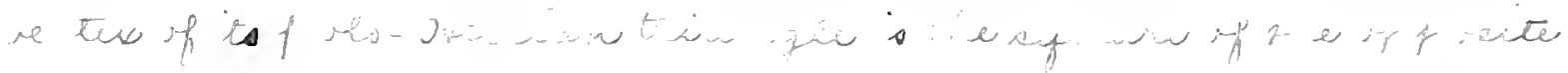
sicue.

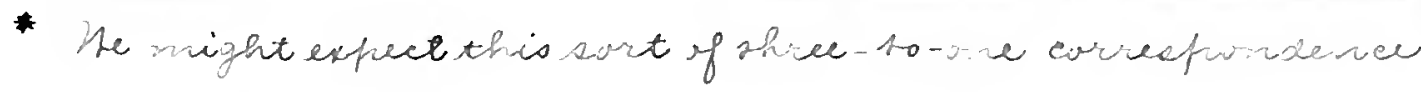

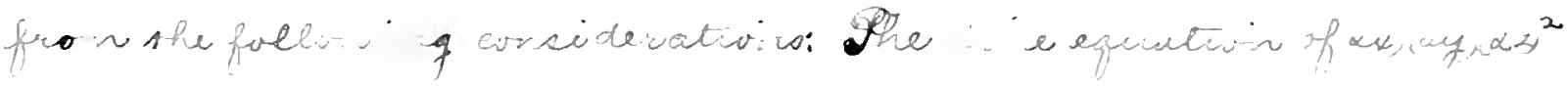

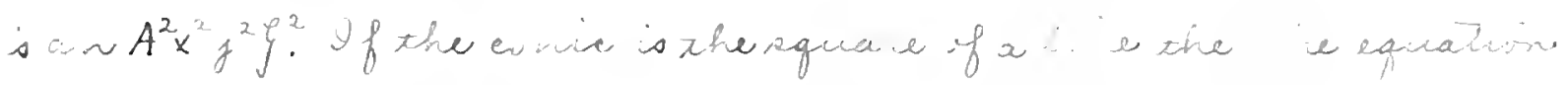

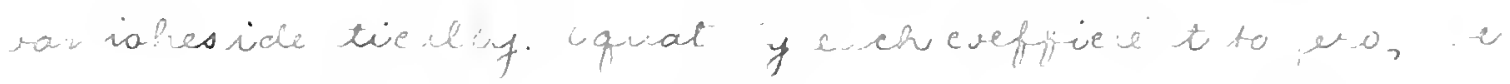

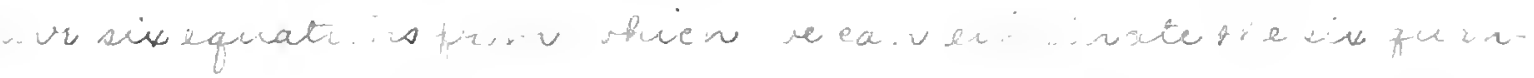

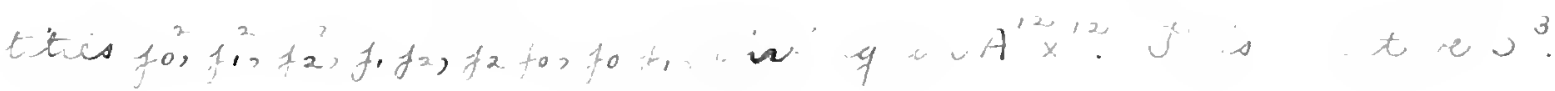




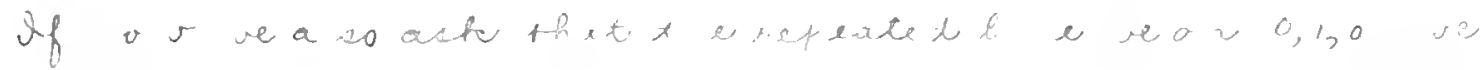
r

$$
b_{2}=y_{1}=0
$$

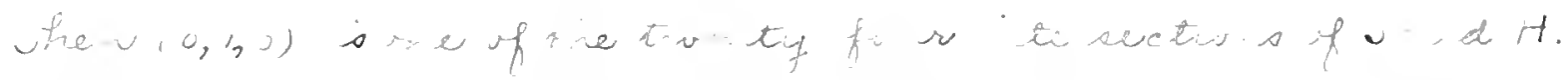

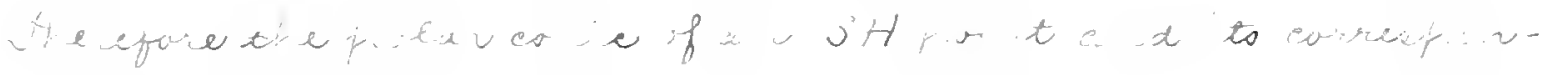

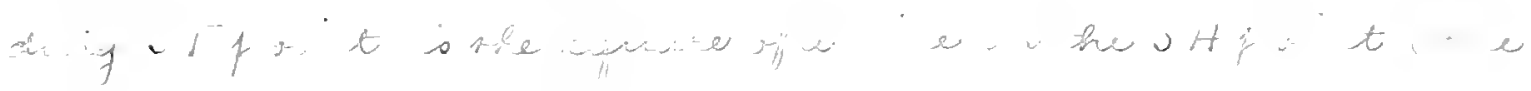

$t=\ldots=t \leq 0$ it -20 .

If f frivalix, we aster that the polar evince of j, is, and $(0,0,1, \text { be } x)^{2}$, we tar

$$
r=b_{2}=f=e_{1}=0 .
$$

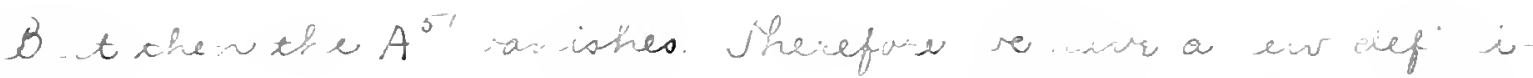

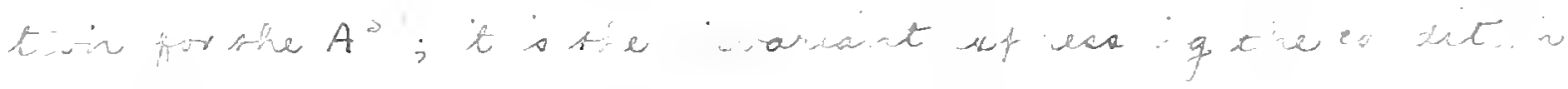

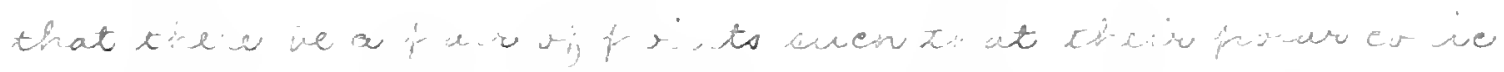

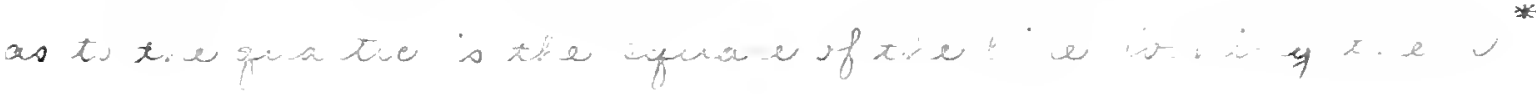

\$12. Salmon's bonner.

Salmon has shown that wish any plane curve as w he the See note 4. 

is associated an $A^{3} x^{2(n 2)} y^{n-2}$, wheck when $x$ is a pront on the ewrer fricks vet the remaining interection of ohe tangent at $x$ is the exver. This conness is exprescible in torns of palars of the otesenan of the eurve itself and the Otesacons of the polar cwives of $x$ as to (ay)? In cace of the greartic che ermext is an

$$
A^{3} x^{4} y^{2} \equiv, 5(h x)^{4}(h y)^{2}-9(h, x)(h, y)^{2},
$$

where (hy) is the Jtessian of the quartie and $(h, y)^{3}$ is she Jteosian of the polar enbie of $x$. Esplicitly

$$
\begin{aligned}
& A^{3} x^{4} y^{2}=\sum^{3}\left[x _ { 0 } ^ { 4 } \left\{y_{0}^{2} \cdot 6\left(a g h-a b^{2}-a_{1}^{2} g-a_{2}^{2} h+2 a_{1} a_{2}, l\right)\right.\right. \\
& +y_{0} f_{1}+4\left(a b_{0} g+a h n-2 a b m-a_{2}{ }^{2} b_{0}-a_{1}^{2} n\right. \\
& \left.+2 a_{1} a_{2} m-a_{1} g h+a_{1} e^{2}\right) \\
& +f_{0} y_{2}+\left(a c_{0} h+a g n-2 a b n-a_{1}^{2} c_{0}+2 a_{1} a_{2} n\right. \\
& -a_{2}^{2}+a_{2}, g h+a_{2}, b^{2} \\
& +f_{1}^{2}\left(a b g-b a_{2}^{2}+2 b_{0}-2 a b_{2} b+a f h-a n^{2}\right. \\
& +2 a_{1} a_{2} b_{2},-a_{1}^{2} f_{1}-x_{1} b_{0} q-a_{1} n_{2}+a_{1} t n \\
& +f_{1} f_{2}\left(a b_{0} c_{0}+2 a b_{2} g+2 a c_{1} h-4 a f l-a m m-2, a_{1} c_{1}\right. \\
& +2 a_{2}^{2} b_{2}+4 a_{1} a_{2},-a_{1} c_{0} h-a_{2} b_{0} q \\
& -a_{1} q \cdots-a_{2}++2 c_{1} \cdot m+2 a_{2}+\cdots \text {, }
\end{aligned}
$$



$+f_{2}^{2}\left(a c h-c a_{1}^{2}+a c_{0} n-2 a c, b+a f g-a w^{2}+2 a, a_{2}, c\right.$,

$$
\left.-a_{2}^{2} f-a_{2} c_{0} h-a_{2} g w+2 a_{2}^{2} 2^{2} w\right)
$$

$+x_{0}^{3} x_{1}\left(f_{0}^{2} \cdot 8\left(a b_{0} g+a h w-2 a b \sim-a_{2}^{2} b_{0}-a_{1}^{2} \sim+2 a_{1} a_{2} \cdots\right.\right.$

$$
\left.-a, g h+a, e^{2}\right)
$$

$+f_{0} f_{1} \cdot 2\left(a b g-b a_{2}^{2}+1 a b u-2 a b_{2} b+a b+4 a n^{2}\right.$

$$
\begin{aligned}
& +2 a_{1} a_{2} b_{2}-a_{1}^{2} f+5 a_{1} b_{0} g-12 a_{2} b_{0} l \\
& -7 a_{1} n+12 a_{2} h n+2 a_{1} b n_{1}-6 h^{2} \\
& \left.+0 n b^{2}\right)
\end{aligned}
$$

$+f_{0} 1_{2}\left(7 a b_{0} c_{0}+2 a b_{2} g+2 a c_{1} h-4 a f l-7 a n n-2 a_{1}^{2} c_{1}\right.$

$$
\begin{aligned}
& -2 a_{2}^{2} b_{2}+4 a_{1} a_{2} f-7 a_{1} c_{0} b-1 a_{2} b_{0} g+17 a_{1} g \pi \\
& +17 a_{2} n=10 a_{1} b x-10 a_{2} b=-12 g h d \\
& \left.+12 b^{3}\right)
\end{aligned}
$$

$+f_{1}^{2} \cdot 3\left(a b n+a b_{0} b+6 a_{1} g-2 a b_{2}=-2 b a_{2} b\right.$

$$
\left.+2 a_{2} b_{2} b-a_{1}+h-b_{0} g h-h^{2} x+2 b l n\right)
$$

$+f_{1} f_{2} a b c_{0}+5 a b_{0} c_{1}-b a_{2} g+3 a b_{2} w-9 a f n$

$$
+2 a_{1} b_{0} c_{0}+1 a_{1} b_{2} q-5 a_{1} c_{1} h-4 a_{2} v_{0}
$$$$
-10 a_{2}, b_{2} b+11 a_{2,},-3 c_{0} u^{2}-2 a_{1} b
$$

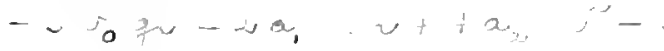





$$
\begin{aligned}
& f_{2}^{2}\left(2 a c b_{0}+2 b_{2} c_{0}-2 c a_{1} v-3 a e_{1} v-2 a_{2} \varphi_{0} e_{0}\right. \\
& -a_{2} b_{2} q+5 a_{2} e_{1} v 1-4 a_{1} e_{0}=v-2 e_{1}, e_{1} b \\
& +a_{1} f q-+z_{2} b-3 c_{0} \cdot b-t a_{1} \cdots+a_{2} a_{2} \\
& \left.-3+2 v+2 i^{2} w\right)
\end{aligned}
$$

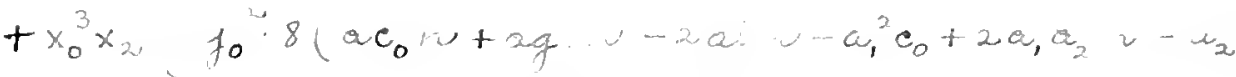$$
-a_{2} \cdot g^{2}+a_{2} t^{2}
$$

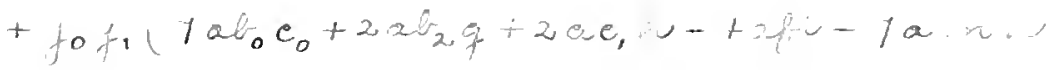$$
-2 a_{1}^{2} e_{1}-2 a_{2}^{2} v_{2}+1 a_{1} x_{2} \gamma-7 a_{1} e_{0}
$$$$
-7 a_{2}, 5_{0} q+11 a_{1} q+17 a_{2},-10 a_{1} x
$$$$
-10 a, b-122 t+12 e^{3}
$$

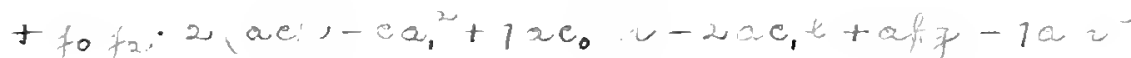$$
+2 a_{1} a_{2} c_{1}-a_{2}^{2} t+5 a_{2} c_{0} v-1 a_{1} a_{1} c_{0}
$$$$
+12 a_{1}+2 v-1 a_{2}+\cdots+2 a_{2}, \ldots+y^{2},
$$$$
\left.+0, x^{*}\right)
$$

$$
\begin{aligned}
& 7 f_{1}^{2}\left(2 a b e_{0}+a b_{0} e_{1}-2 b x_{2} q-3 x b_{2}, 2 a_{1} e_{0} e_{0}\right. \\
& +5 a_{1} s_{2}+a_{1} a_{1} J+4 a_{2} v_{0}-2 a_{2} b_{2} b
\end{aligned}
$$

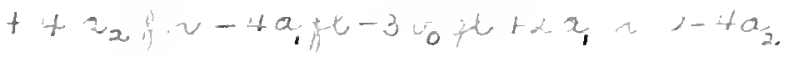

$$
\begin{aligned}
& -3 \ldots,+2,2 \text {, }
\end{aligned}
$$

$+f_{1} f_{2}\left(a c b_{0}+5 a b_{2} c_{0}-c a_{1} \ldots+3 a c_{1} w-9 a b v\right.$ 

40

$$
\begin{aligned}
& +2 a_{2} f_{0} c_{0}-5 a_{2}, b_{2} g+7 a_{2} c_{1} h, 4 a_{1} c_{0} x \\
& -10 a_{1} c_{1} l+11 \omega_{1} \lg -3 \log ^{2}-2 a_{2} f l \\
& -3 c_{0} k+4 a_{1} n^{2}-2 a_{2}+2-3 q k, \\
& \left.+3 \ldots k n+, b^{2} n\right) \\
& +f_{2}^{2} \cdot 3(a c)+a c_{0} b+c a_{2} v-x a c_{1} v-2 c_{1} v+2 a_{1} e_{1} \\
& \left.-a_{2} f y-e_{0} \min -q^{2} 2+2 a b j\right\}
\end{aligned}
$$

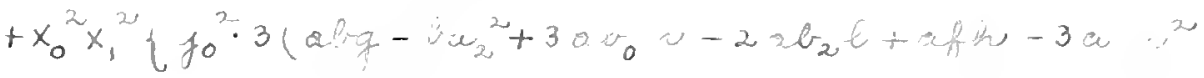

$$
\begin{aligned}
& +2 a_{1} \cdot a_{2} b_{2}-a_{1}^{2} 1+a_{1} r_{0} a^{2}-4 a_{3} b_{0} z
\end{aligned}
$$

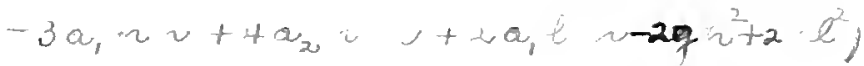

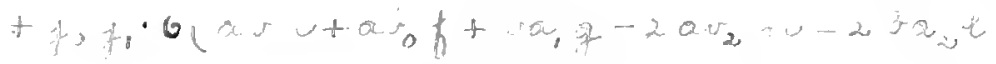

$$
\begin{aligned}
& +2 a_{2}, r_{2},++a_{1}=1-v_{1}-1-v_{0}, \\
& -+a_{1} v^{2}-40 c^{2}-5 i^{2} v+k b
\end{aligned}
$$

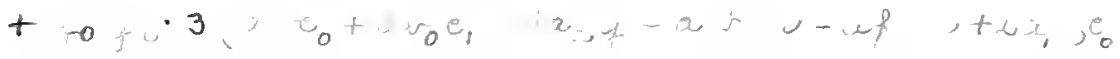

$$
\begin{aligned}
& +32, s_{2}-2, e_{1},+\ldots, \ldots, \ldots, 2, \ldots \\
& +3 a_{2}-1-3 c_{0} \cdot v^{2}-2 a, b l-7 b_{0} \cdot b \\
& -2 a_{1} \ldots,-+a_{2} \cdot v^{x}+5 q,-5 l v \\
& +00^{2} \quad 1 \\
& +f_{1}^{2} \cdot 3\left(x b f-a b_{2}^{2}+3<a_{1} v-\infty u_{2} v+\cdot 4 h-3 v^{2}\right. \\
& +2 a_{2}+2+4_{0} f-0^{2} q-4 a, 2,
\end{aligned}
$$



$+1$

$$
\begin{aligned}
& -3 b_{0} n,+4 b_{2} h b^{2}+2 b_{0} t=-2 f^{2} \\
& +2 \sim v^{2} \\
& +f_{1} f_{2} ; 3\left(2 b_{1}+b_{0} c_{0} a b_{2} f-a_{2} v j=b+2 a_{1} b_{0} c_{1}\right. \\
& +3 a_{2} b_{0} b-v_{0} c_{0} t+4 a_{1}, \ldots-2 a_{2,}, \ldots \\
& +3 w_{2}+2+3 c_{1} v^{2}-1 a_{1} \rho,-2,+q w \\
& -2 v_{0} l,-+b_{2} b^{2}+5 t: l-2 \sim, \\
& \left.+0 a^{3}\right) \\
& +f_{2}^{2}\left(a b e-x b_{2} e_{1}-a_{2} c_{0}+2 c a_{1} v_{0}-3 e n^{2}+4 a_{1} v_{2} c_{0}\right. \\
& +4 z_{2} v_{0} e_{1}-0 a_{1} c_{1} v-0 i_{c} c_{0} t-3 a_{2} \& 2
\end{aligned}
$$

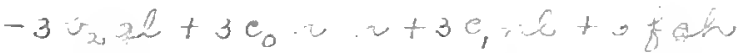

$$
\begin{aligned}
& -3 k^{2}-3 g 2^{2}-, \cdots 2^{2}+12,2, j \\
& +x_{0}^{2} x_{1} x_{2},{ }_{0}^{2} \cdot 3,32 b_{0} e_{0}+2 a v_{2}+2 a c_{1} v-+a-b-3 x \\
& -\alpha x_{1}^{2} c_{1}-2 x_{2}^{2}-x++a_{1} x_{2}+3 x_{1} e_{0}
\end{aligned}
$$

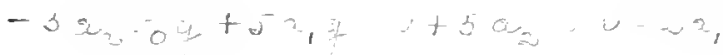

$$
\begin{aligned}
& -2 x_{2} \ldots,+1=\cdots+ \\
& +0 f_{1} \cdot 3 \times e_{0}+3 s_{0} e_{1}-e_{2}, t+2,3 f \\
& t, e_{0}+5, \ldots-3 x_{1},+4
\end{aligned}
$$

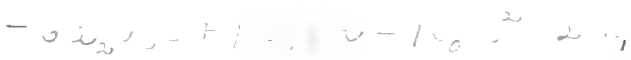

$$
\begin{aligned}
& 1 .+\cdots+1,2+1 \\
& +
\end{aligned}
$$



$1+2$

$$
\begin{aligned}
& +f_{0} f_{x} \cdot 3, a c_{0}+3 a b_{x} c_{0} c_{1} n+a c_{1}, 5 a h^{2} \\
& +a_{2}, \hat{r}_{0} e_{0} \quad 3 a_{2} b_{2} q+3 a_{2}, h+4 a_{1} c_{0} \ldots \\
& -v a_{1} c_{1}+7 a_{1} f-7 b_{0} q^{2} \quad 2 a_{2} h
\end{aligned}
$$

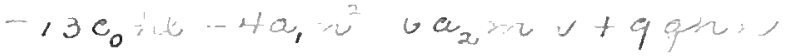

$$
\begin{aligned}
& +2+100^{2}+1
\end{aligned}
$$

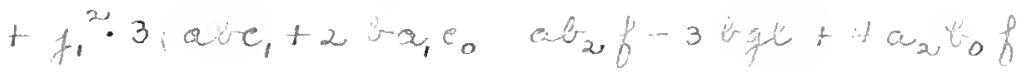

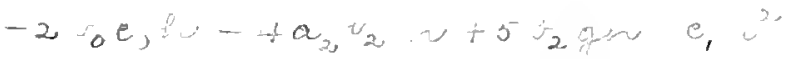

$$
\begin{aligned}
& -2 a, k-2 s_{0} q \cdots+4 k \\
& +f_{1} x_{2} a b c+8 a b_{2} c_{1}+2 b_{a_{2}} c_{c}+2 c a_{1} v_{0}-9 a f^{2}-3 g^{2} \\
& -a p h^{21}+10 a_{1} b_{2} c_{0}+10 a_{2} b_{0} e_{1}-12 a_{1} c_{1}, 2 \\
& -12 a_{2} v_{2},-2,2,2-3 a_{2}, h^{2} \\
& -180, z-12 e_{2}, l-1, c_{0}, \quad \\
& -12 e, w+30 t+2+6 y^{2}+0^{2} \\
& +24 t=1 \\
& +f_{2}^{2} \cdot 3\left(a b_{2}+2 b a_{0}-a c_{1} f-3 \cdot k+k a_{1} e_{0} f\right. \\
& -2 e_{0} e_{0}+a_{i}, w-b_{2}+3 c, q 2 \\
& \left.\left.-2 a_{2} h, 2 e_{0}, \quad f+k+i t u^{\prime}\right)\right]
\end{aligned}
$$

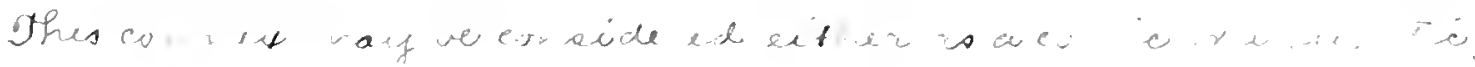

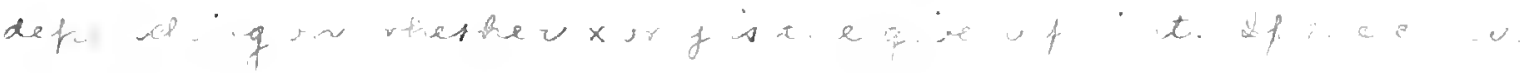



4.3

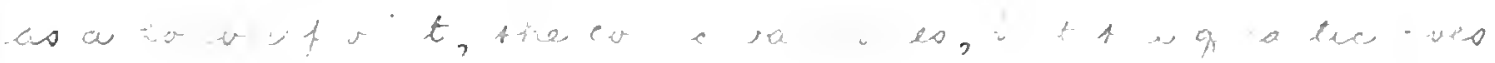
t. . t

$x-a_{1}=a_{2}=0_{2}$

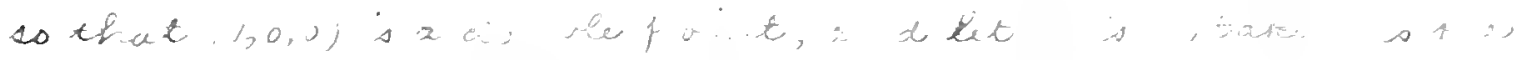

$\gamma: t^{2} f \cdot a$

$$
\begin{aligned}
& A^{3} x^{4} y^{2}=x_{0}{ }^{2} x_{1}^{2} \cdot 3\left(-2 x^{2}+2: e^{2}\right) \\
& \left.+x, x^{2} x, x_{2}, 3-4 \quad+4+2\right) \\
& +x_{0}-x^{2}+3-2 q^{2}+2+22^{2}, \\
& \left.+x_{3} x_{i} \cdot 3(-0,-)^{2} v+2 n \ell-v\right) \\
& \left.+x_{0} x_{1}^{2} x_{2} \cdot 3-e_{0} h^{2}-2 \dot{b}_{0} g l-q h n++l^{2} n\right) \\
& +x_{0} x_{1} x_{2}^{2} \cdot 3\left(-b_{0} g^{2}-2 c_{0} h l-q h \cdot+4 b^{2}-1\right) \\
& \left.+x, x_{2}{ }^{3} \cdot 3-c_{0} q h-g^{2} n+2 g l v\right) \\
& \left.+x_{1}^{4} j g h-4 b b^{2}-b_{0}^{2} g-b_{0} \pi n+2 b_{0} l a\right) \\
& +x_{1}^{3} x_{2}-b_{0} e_{0} t+b_{2} g k-+y_{0} g u+2 b_{0} l u-1+b_{2} e^{2}-3 n=2 x \\
& \left.+, l_{2}, 2^{x}\right) \\
& +x_{1}^{2} x_{2}^{2}\left(-3 b_{0} q u-3 e_{0} h m+3 t g h-0 g e^{2}-3 q n^{2}-3 x u^{2}+12 k m x\right) \\
& +x_{1} x_{2}^{3} \quad b, e_{0} q++e_{1} g h-4 e_{0} n \sim+2 e_{0} l \quad v-4 e_{1} z^{2}-3 q x \\
& +\ldots v^{2} ; \\
& +x_{2}+\left(e q c-+c l^{2}-c_{c}^{2}=c-c+i+2 c_{0} l-u\right) \text {. }
\end{aligned}
$$



$4+1$

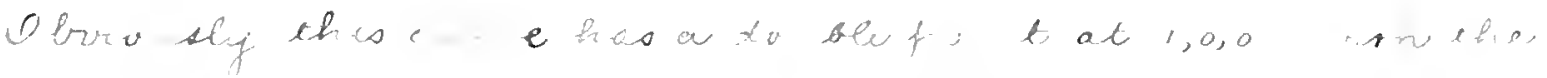

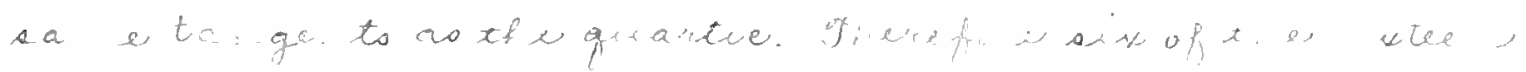

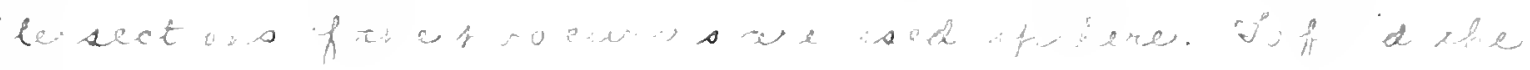

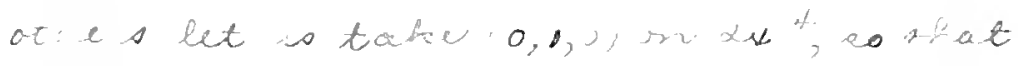

$$
b=j \text {. }
$$

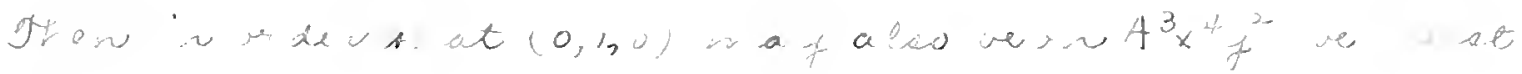

$\therefore$. in

$$
v(b, q+h v-2 l \cdots j-0 .
$$

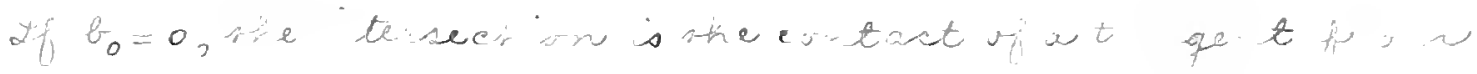

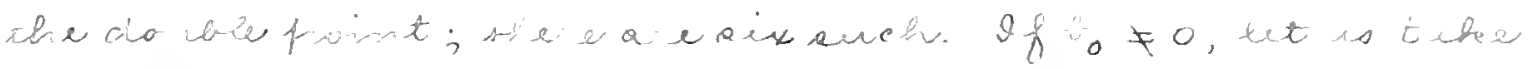

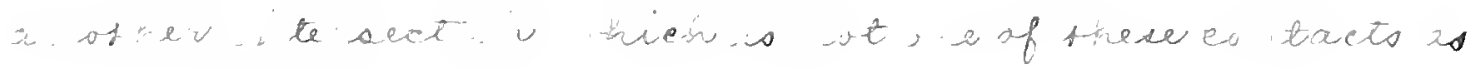
$(0,0,1 \ldots$ Yi,

$$
\begin{gathered}
e=0, \\
b, g+h-2-2 b \cdot r=0, \\
e_{0} u+g-2 i \cdot v=0 .
\end{gathered}
$$

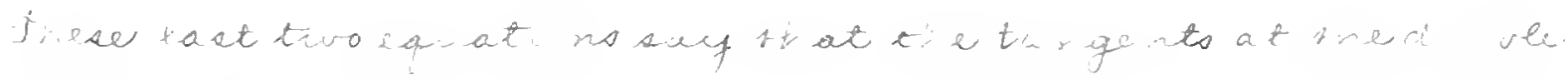
forit, qiven by

$$
x_{1}^{2}+2 l x_{1} x_{2}+x_{2} x_{2}-y_{2}
$$

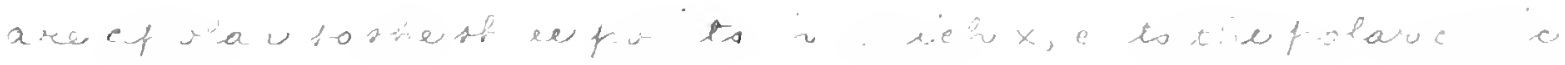



$1 / 3$

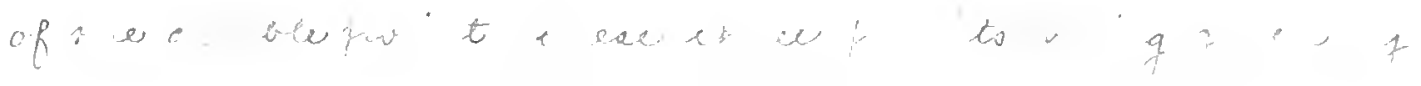

$$
x_{0} y_{2}^{3}-3, v y_{1} y_{2}+3 \cup y_{1}^{2} \xi_{2} \cdot c, g_{1}^{3}-0 .
$$

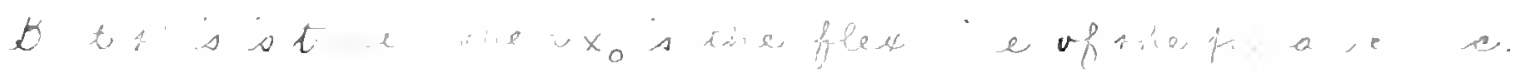

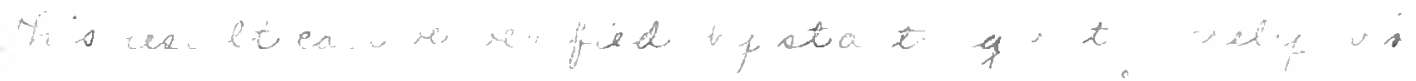

$$
r=a_{1}=a_{2}=0
$$

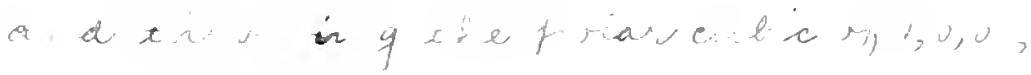

$$
3 x_{0} x_{1}^{2}+0 x_{0} x_{1} x_{2}+3 q x_{0} x_{2}^{2}+b_{0} x_{1}+32 x_{1}^{2} x_{2}+32 x_{1} x_{2}^{2}+e_{2} x_{2}^{3}
$$

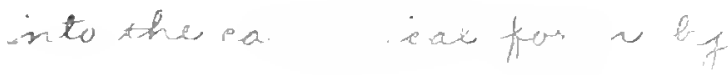

$$
\text { r }-q=r=-\therefore-0 \text {. }
$$

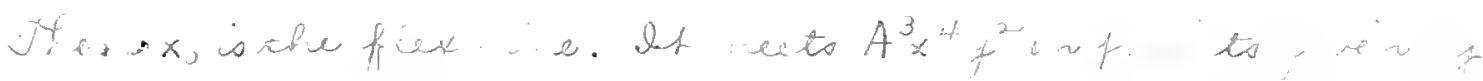

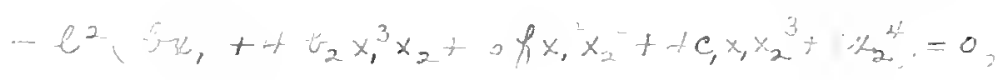

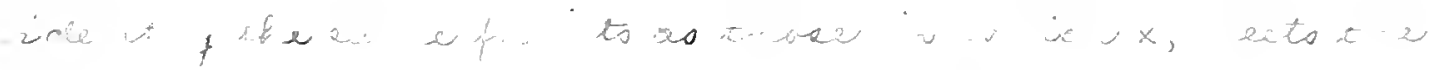

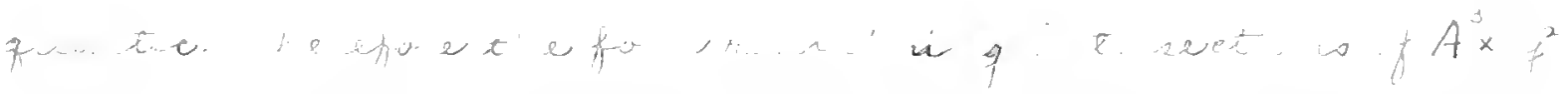

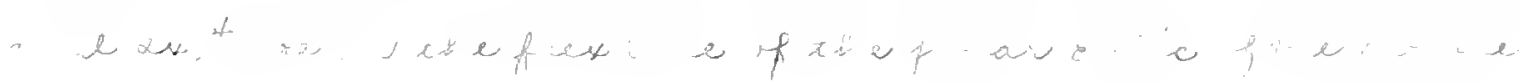
$f \cdot i$

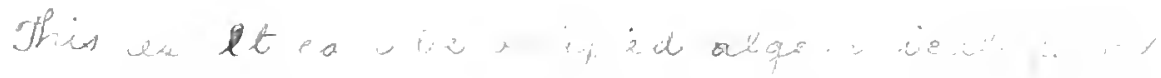

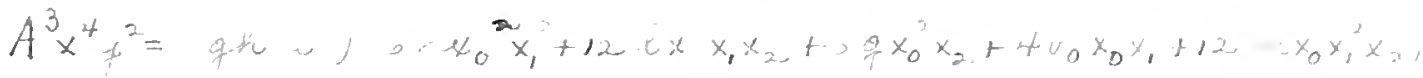

$$
\begin{aligned}
& +12+x_{0} x_{1} x_{2}^{2}+2+c_{0} x_{2} x_{2}+x_{1}+x_{2} x_{1} x_{2}+2 x_{1} x_{2} \\
& ++c_{1} x_{1} x_{2}+e_{1} x_{2}
\end{aligned}
$$





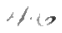

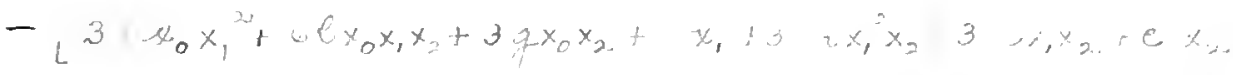

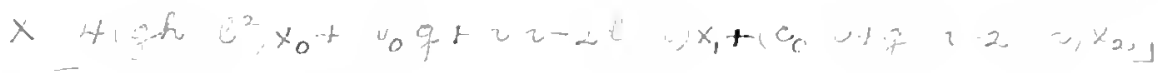

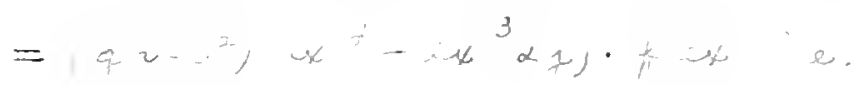

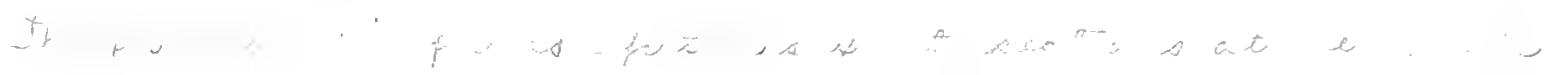

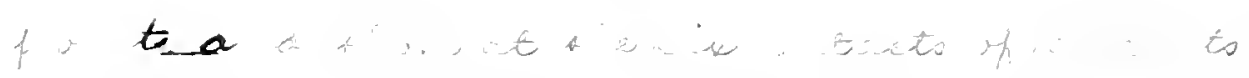

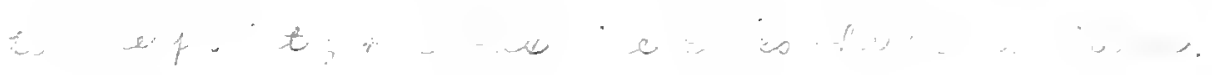

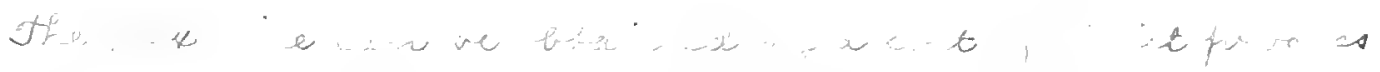

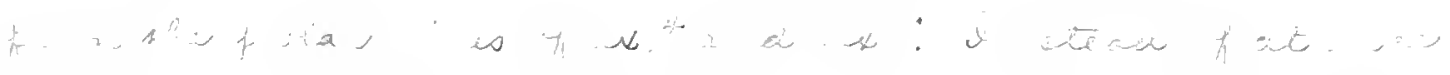

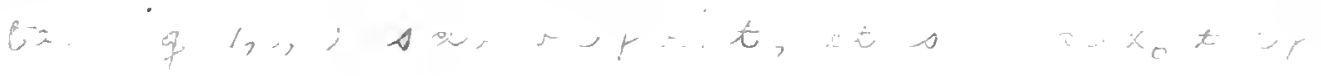
thi, 20,2 thot t t t

$$
2,-a_{2}=c
$$

$$
\begin{aligned}
& -f+\infty \quad, c, \ldots
\end{aligned}
$$

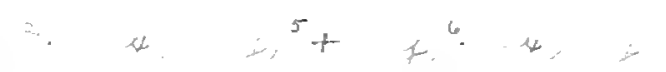

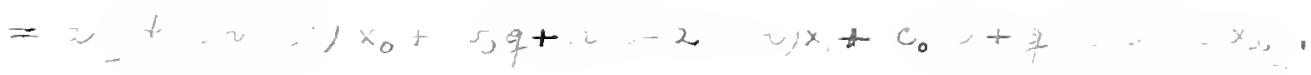

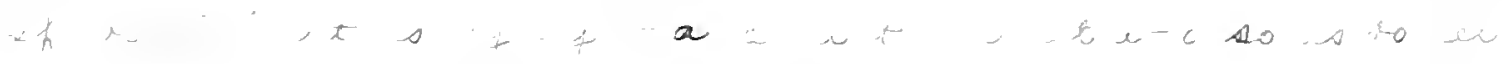

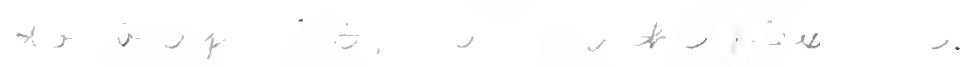

$$
\begin{aligned}
& A^{3} x^{+} x^{2}+\text { t } \\
& \text { in to to to to to to }
\end{aligned}
$$



17$$
x^{5}=3,0 \times,+, \ldots
$$$$
+\frac{1}{+} x_{0} x_{2} x_{1}
$$$$
x+x^{2}-2
$$

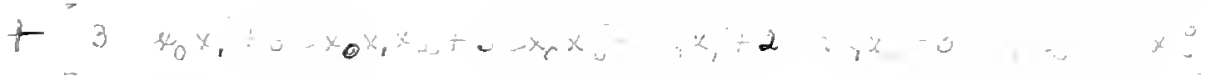$$
x \quad x_{0}^{2} \cdot-1 C(\ldots,-)
$$

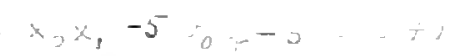$$
x+2,-3 ;, 2-3 \div
$$$$
\therefore i_{i}-
$$$$
x, \therefore, \quad, s_{0}
$$$$
=
$$$$
=t, \quad-x_{c} \text {. }
$$ 


$$
\bullet
$$


48

3

1

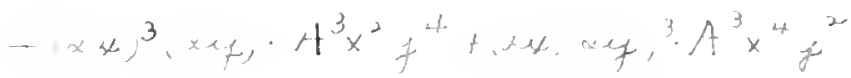

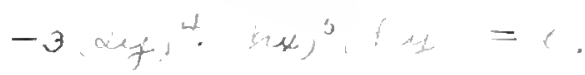



49

Note 1.

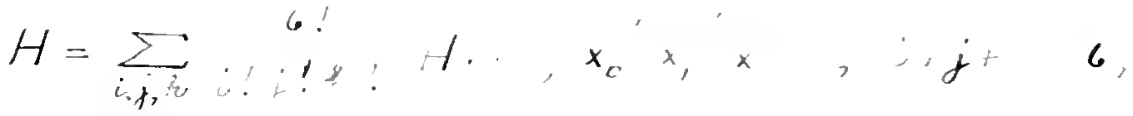

$$
\begin{aligned}
& H_{600}=a g h-a l^{2}-a_{1}^{2} g-a_{2}^{2} h+2 a_{1} a_{2} l, \\
& H_{5,0}={ }_{3}^{1}\left(a b_{0} g+a h n-2 a l m-a_{2}^{2} b_{0}-a_{1}^{2} n+2 a_{1} a_{2} m\right. \\
& \left.-a_{1} g h+a_{1} e^{2}\right) \text {, }
\end{aligned}
$$

$$
\begin{aligned}
H_{501}=\frac{1}{3}\left(a c_{0} h+a g m-2 a l n-a_{1}^{2} c_{0}+2 a_{1} a_{2} m-a_{21}^{2} m\right. \\
\left.-a_{2} g h+a_{2} l^{2}\right)_{2}
\end{aligned}
$$

$$
\begin{aligned}
H_{420}=\frac{1}{15}(a b g & -b a_{2}^{2}+4 a b_{0} n-2 a_{2} l+a f h-4 a m n^{2} \\
& +2 a_{1} a_{2} b_{2}-a_{1}^{2} f+2 a_{1} b_{0} g-b a_{2} b_{0} l-4 a_{1} h n \\
& \left.+b a_{2} h m+2 a_{1} b_{2 n}-3 g h^{2}+3 h l^{2}\right),
\end{aligned}
$$

$$
\begin{aligned}
& H_{402}=\frac{1}{15}\left(a c h-c a_{1}^{2}+4 a e_{0}{ }^{2}-2 a c_{1} l+a f g-4 a x^{2}\right. \\
& +2 a_{1} a_{2} c,-a_{2}^{2} f+2 a_{2} c_{0} f u-0, a_{1} c_{0} b+0 a_{1} q 2 \\
& \left.-4 a_{2} g+2 a_{2} t n-3 g^{2} n+3 g t^{2}\right),
\end{aligned}
$$

$$
\begin{aligned}
& H_{411}=\frac{1}{15}\left(2 a b_{0} c_{0}\right.+a b_{2} g+a c_{1} h-2 a f l-2 a m w-a_{1}^{2} c_{1}-a_{2}^{2} b_{2} \\
&+2 a_{1} a_{2} f-2 a_{1} c_{0} h-2 a_{2} b_{0} g+4 a_{1} g \cdots \\
&\left.+4 a_{2} h n-2 a_{1} l_{n}-22_{2} l_{m}-3 g h b+3 b^{3}\right), \\
& H_{330}=\frac{1}{10}\left(a b n+a b_{0} f+b a_{1} g \text { ab } b_{2} m-2 b a_{2} b+2 a_{2} b_{2} h\right.
\end{aligned}
$$



50

$$
\begin{aligned}
& +2 a_{1} b_{0} n \quad a_{1} b h-b_{0} g h-2 a_{1}=n^{2} 2 b_{0} b^{2}
\end{aligned}
$$

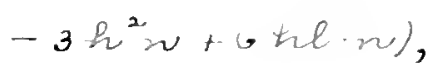

$$
\begin{aligned}
& H_{321}=\frac{1}{30}\left(a b c_{0}+2 a b_{0} c_{1}-b_{1} a_{2} g-3 a f m+2 a_{1} b_{0} c_{0}+4 a_{1} b_{2} g\right. \\
& -2 a_{1} e_{1} b+2 a_{2} b_{0} \sim-4 a_{2} b_{2} l+3 a_{2} f h \\
& -3 c_{0} h^{2}-2 a_{1} f l-6 b_{0} g l-2 a_{1}, m n-2 a_{2} m^{2} \\
& +3 g h m+6 l^{2} m / 2 \\
& H_{312}=\frac{1}{30}\left(a c b_{0}+2 a b_{2} c_{0}-c a_{1} h-3 a f w+2 a_{2} b_{0} c_{0}-2 a_{2} b_{2} g\right. \\
& +4 a_{2} c_{1} h+2 a_{1} e_{0} m-4 a_{1} c_{1} l+5 a_{1} f g \\
& -3 b_{0} g^{2}-2 a_{2} f l-6 c_{0} h l-2 a_{1} \nu^{2}-2 a_{2} m w \\
& +3 g h+6 l^{2}+0,2 \\
& H_{222}=a_{0}^{\prime}\left(a b c+2 a b_{2} c_{1}+2 b a_{2} c_{0}+2 c a_{1} b_{0}-3 a f^{2}-3 b g^{2}-3 c h^{2}\right. \\
& +10 a_{1} b_{2} c_{0}+10 a_{2} b_{0} c_{1}-6 a_{1} c_{1}=0 a_{2} b_{2}= \\
& -6 b_{0} c_{0} l-6 a_{1} h=-6 a_{2} h m-6 b_{0} g w \\
& -6 b_{2} g l-6 c_{0} h m-0 e_{1} h l+18 f g h+18 b i n g .
\end{aligned}
$$

The otheic coeffieie nts nay be vbta hed fio v these bf syp. itsy. 

51

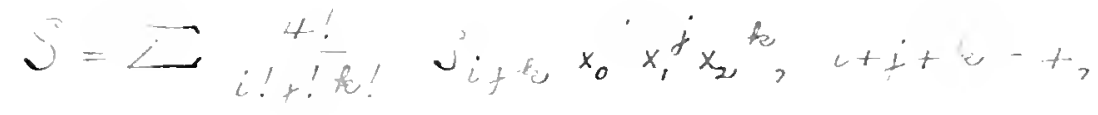

AS:C

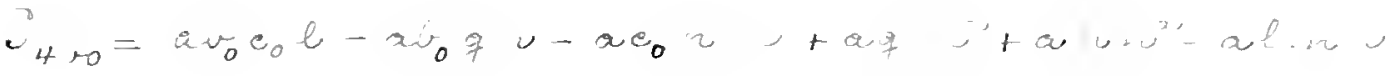

$$
\begin{aligned}
& -a_{1} x_{2} b_{0} c_{0}+a_{1}^{2} e_{0},+a_{2}^{2} b_{0},+a_{1} b_{0} q^{2}+a_{2} c_{0} 2^{2}
\end{aligned}
$$

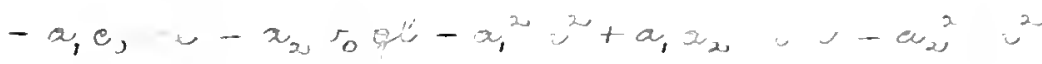

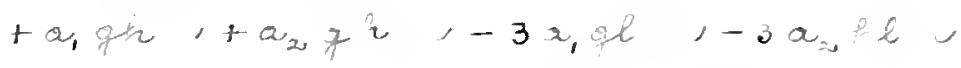

$$
\begin{aligned}
& +2 a_{1} b^{2} u+2 a_{2} b^{2} v-g^{2} u^{2}+2 a b^{2}-b^{4},
\end{aligned}
$$

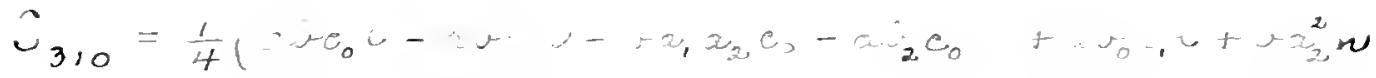

$$
\begin{aligned}
& -2 y_{0}+b+b a_{1} g^{2}+2 a b_{2} g m-a c_{1} h m-b a_{2} g l \\
& -a b_{2} l w+2 a f h w-a f b^{2} w+a_{1}^{2} b_{2} c_{0}-a_{1} a_{2} b_{0} e_{1} \\
& +a_{2}^{2} b_{0} f+a_{2} b_{0} c_{0} h+a_{1}^{2} c_{1} m+a_{1} a_{2} b_{2} n-2 a_{2}^{2} b_{2} m \\
& -a_{1} b_{0} c_{0} b+a_{2} b_{2} g h+a_{2} c_{1} h^{2}-2 e_{1}^{2} b v+a_{1} a_{2} f \cdots \\
& +2 a_{1} b_{0} g n^{\prime}-3 a_{1} b_{2} g l-a_{1} e_{1} h l+2 a_{2} b_{2} l^{2}-2 a_{2} b_{0} b \\
& +a_{1} f g h-b_{0} g^{2} h-3 a_{2}, f h l+2 a_{1} f b^{2}-2 a_{1} g m^{2} \\
& -a_{2} h m n+b_{0} g l^{2}+a_{1} f m \cdots+2 a_{2} k n^{2}-g h^{2} \cdots \\
& +2 q h l: u+b e^{2} n-2 l^{3} \cdot u \text { ) } \\
& \dot{j}_{301}=\frac{1}{4}\left(a c b_{0} b-a c h+c a_{1} a_{2} b_{0}-2 b_{0} e_{1} q+a b_{20} c_{0}+c a_{1}^{2} 2\right.
\end{aligned}
$$





$$
\begin{aligned}
& -a c_{0} f_{1}+c a_{2} b^{2}-a b_{2} q{ }^{2}+2 x_{1}, \ldots a_{1}
\end{aligned}
$$

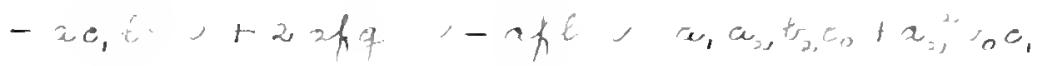

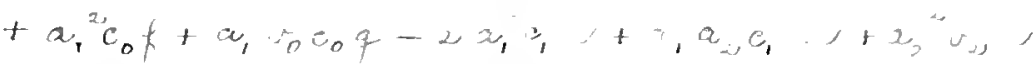

$$
\begin{aligned}
& -a_{2} v_{0} e_{0} b+a_{1} r_{2} z^{2}+u_{1} c_{1} v+a_{1} a_{3}+\cdots a_{2}^{2} \\
& -a_{2}, y_{1} l+2 a_{2} c_{0}{ }^{2},-3 a_{2} e_{1},-2 a_{1} e_{0} t, \\
& +2 a, c_{1}+a_{2, \pi^{2}}+c_{0} a^{2}-3 x_{1}=b^{2}-a_{1} \\
& +2 a_{2}+b^{2}-2 a_{2}, \cdots+c_{0} b^{2}+2 a_{1}, \cdots+2,2^{2}=
\end{aligned}
$$

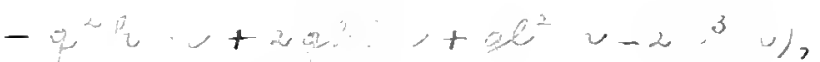

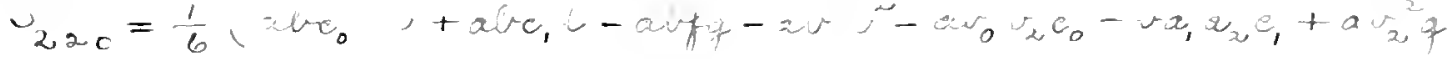

$$
\begin{aligned}
& -2 v_{2} c_{1} \cdot u+r a_{2}^{2} \hbar-r a_{2} e_{0} v+2 j_{0} k \cdots-z_{2}^{2}+b
\end{aligned}
$$

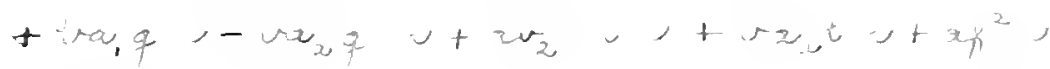

$$
\begin{aligned}
& +v y^{2}-a f \ldots+b^{2}+u_{1}^{2} r_{2} c_{1}-a_{2}^{2} v_{1}^{2}+a_{2} r_{0}^{2} c_{0}
\end{aligned}
$$

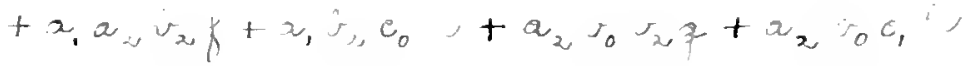

$$
\begin{aligned}
& -a, j_{0} c_{0} \cdot \cdots a_{1} j_{0} c_{1},-x, b^{2}-j_{0}^{2} 2^{2}-x_{2}+
\end{aligned}
$$

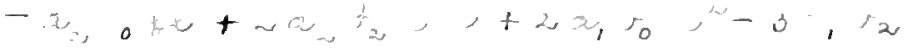

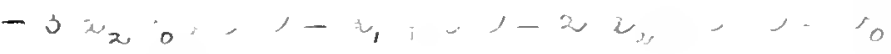

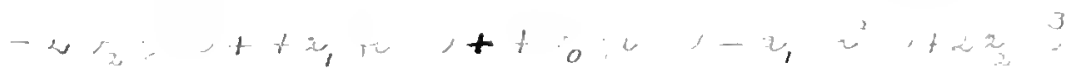

$$
\begin{aligned}
& -5_{0}, 2,+2,3+\ldots, \ldots, \ldots,- \\
& +7 \ldots,-3,2,2
\end{aligned}
$$



53

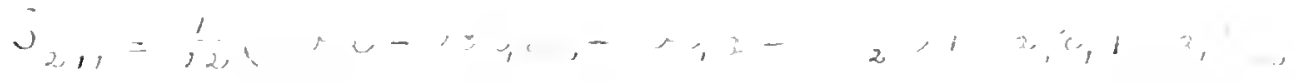

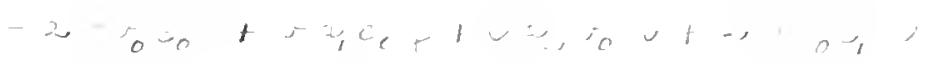

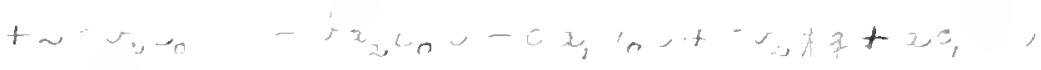

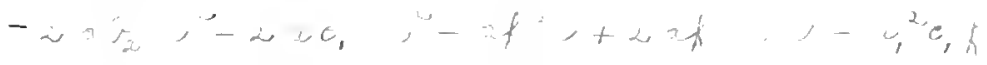

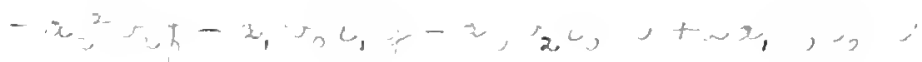

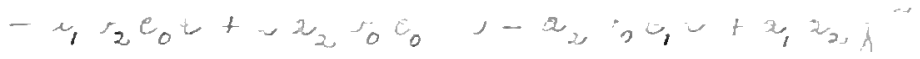

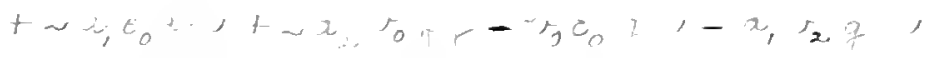

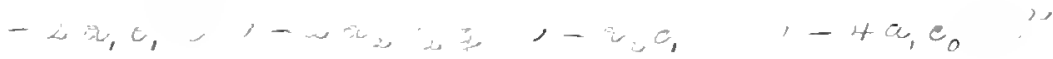

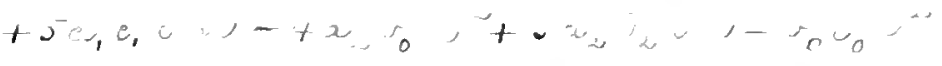

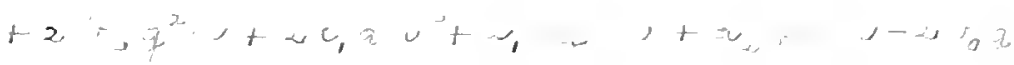

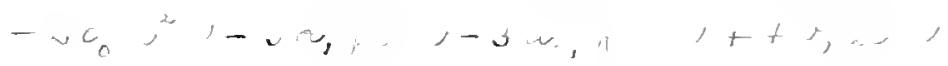

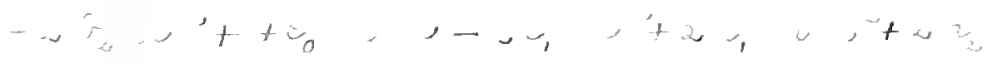

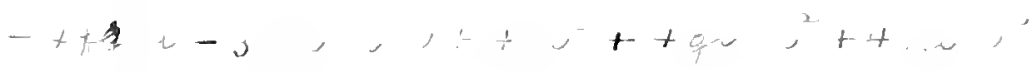

$$
\begin{aligned}
& -1 i^{2}, 1 .
\end{aligned}
$$





\section{Note 3}

The corfficurt of $x_{0}{ }^{6}$ in $T$ is votained from the supression given by Sabmon for the mariant I of the eubie as

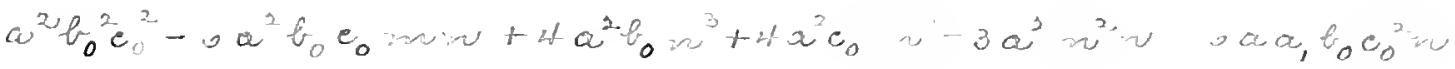

$$
\begin{aligned}
& -6 a a_{2} b_{0}^{2} e_{0} g+0 a a_{1} b_{0} c_{0} g=n+0 a a_{2} b_{0} e_{0} b=1+12 a_{1} a_{1} b_{0} c_{0} b_{n} \\
& +12 a a_{2} b_{0} c_{0} b n+4 a b_{0}^{2} g^{3}+4 a c_{0}^{2} h^{3}+12 a b_{0} e_{0} g h b+18 a a_{1} c_{0} h m i v \\
& +18 a a_{2} b_{0} g a n-12 a a_{1} b_{0} g n^{2}-12 a a_{2} c_{0} b n^{2}-20 a b_{0} c_{0} b^{3} \\
& -24 a a_{1} c_{0} b n^{2}-24 a a_{2} y_{0} b^{2}-12 a b_{0} g^{2} h \cdot v-12 a c_{0} g h^{2}= \\
& -24 a b_{0} g^{2} l=-2+a e_{0} h^{2} b^{2}+60 a_{1} g n^{2} n-12 a a_{1}, \ldots \\
& -12 a a_{2} g n^{3}+6 a a_{2} h: n n^{2}+36 a b_{0} g l^{2} n+36 a c_{0} R b^{2}= \\
& +12 a a_{1} b n^{2}+12 a a_{2} b n^{2} n+24 a g^{2} n n^{2}+24 a g h^{2} n^{2} \\
& \text { - } 60 \text { aqhinintinaql } x^{2}+12 a b^{2} a^{2}+12=b^{3} n \omega \\
& +4 a_{1}^{3} b_{0} e_{0}^{2}+4 a_{2}^{3} b_{0}^{2} e_{0}-12 a_{0}^{2} a_{2} b_{0} c_{0} w-12 a_{1} a_{2}^{2} b_{0} e_{0} \\
& +18 a_{1} a_{2} b_{0} e_{0} q^{h}-3 a_{1}^{2} e_{0}^{2} \omega^{2}-3 a_{2}^{2} b_{0}^{2} y^{2}-2+a_{1}^{2} e_{0} e^{2} \\
& 2+a_{2}^{2}, c_{0} \quad 12 a_{1} c_{0} 2 \sim+2+i_{1}^{2} a_{2} c_{0}^{2}+2+a_{1} a_{2} \\
& -12 i_{2}-0 \sim J+3+a_{1} a_{2} \cdot e_{0} e^{2}+2+a_{1} a^{2}+0 a_{1}^{2} c_{0}
\end{aligned}
$$

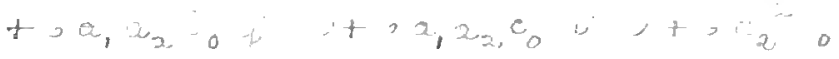

$$
\begin{aligned}
& +12,2,{ }^{2} c_{0}
\end{aligned}
$$



33

$$
\begin{aligned}
& +12 a_{1} e_{0}=1+12 a_{2} \quad v+2, \ldots, \quad 3, a \\
& -12 a_{1} a_{2} \quad 2+a_{2}-12 a_{1} a^{2}-12 a_{2} c_{0}+12 a_{1} c_{0}
\end{aligned}
$$

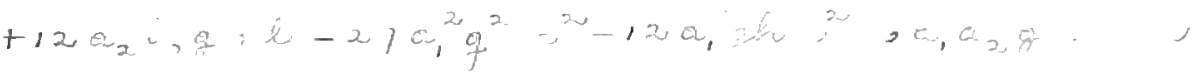

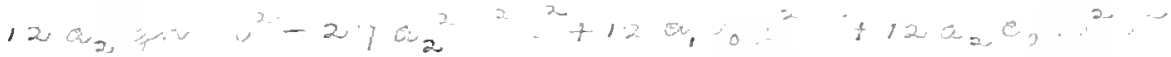

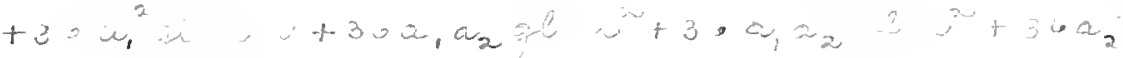

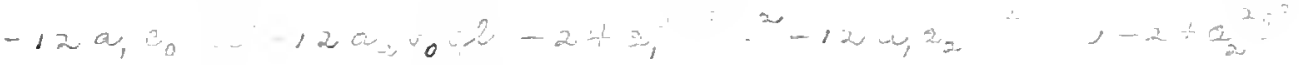

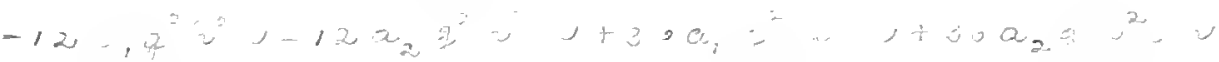

$$
\begin{aligned}
& -1+x_{1} a^{2}-12 a_{2}+i^{2},-36 a_{1} g b^{3} v-36 a_{2} l^{3}
\end{aligned}
$$

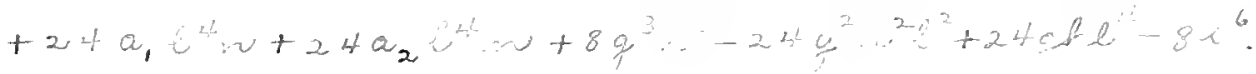

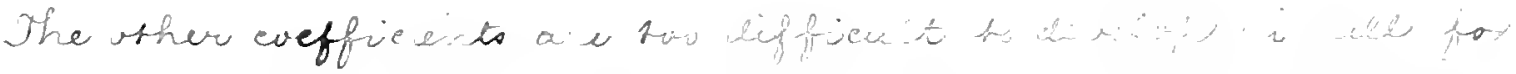

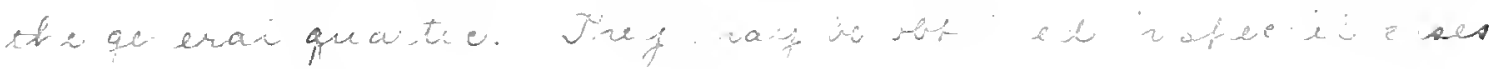

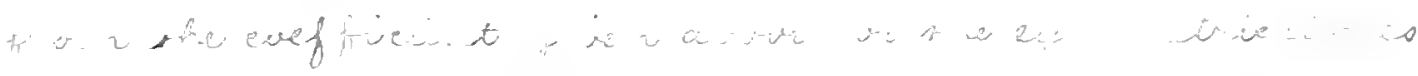

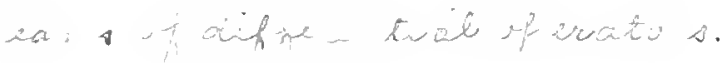



Note 4

Certain invariants of the quartic have a vell-lefined geometrical nearing. The two simplest warets, the $A^{3}$ a $2 A^{6}$ hewers afolarity meanings and ac be included in the lest by courtesy then comes the $A^{15}$ of Dr. Coble, which is the canclition that the quartic be

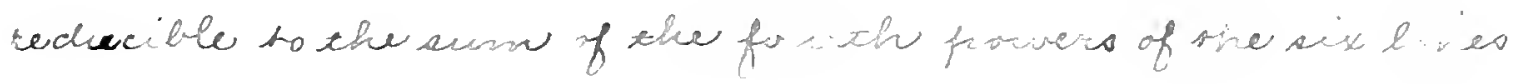
of a complete quadrilateral on what the curaricut s become, t to conies; the discrivinima $t, A^{27}$; the $A^{42}$ of fr. Oramsen,

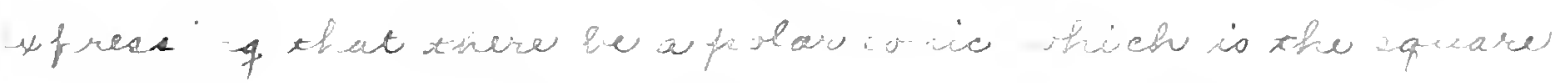

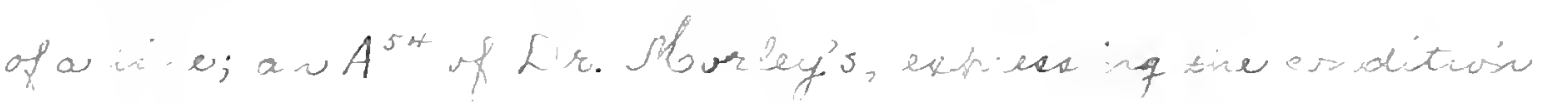
that she qua tie pass the w tree re ties if $x$. e. tough, the

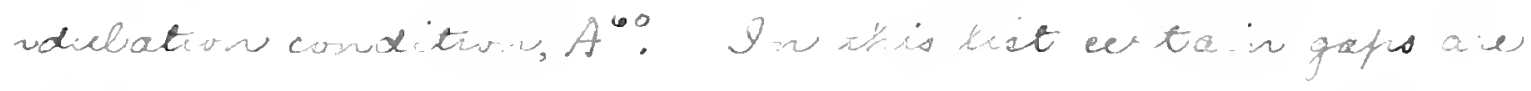

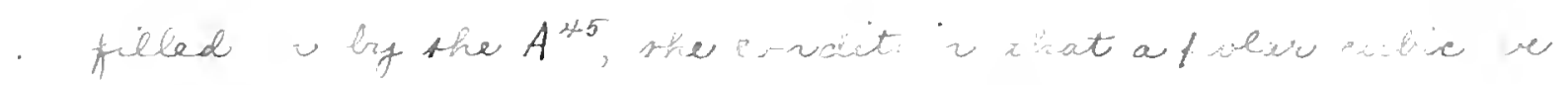

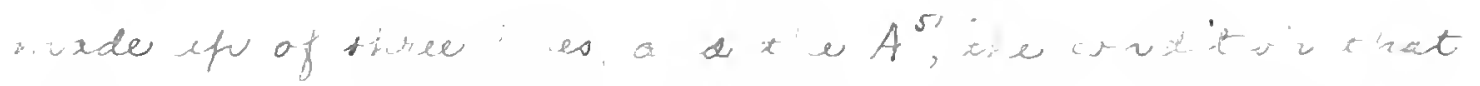

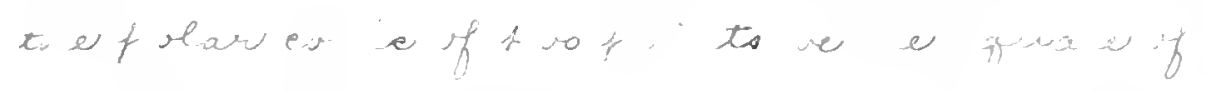

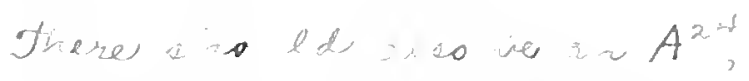

Ar re tour. Hath, wo l XXXI, p.357 (1909) 

, as tivetit.

$\infty, 1,+\infty$ is 2

$f_{0}: P_{2}$

$A^{\prime} J^{\prime}, A$

$\checkmark$

$A \cdots j^{\prime}$

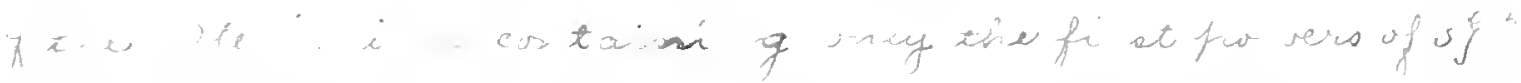

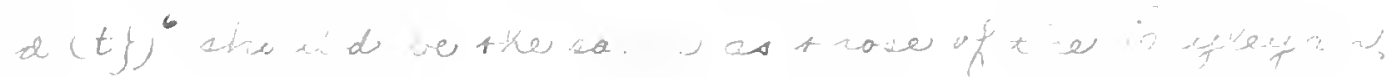

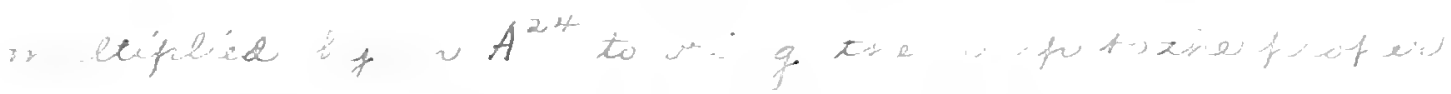

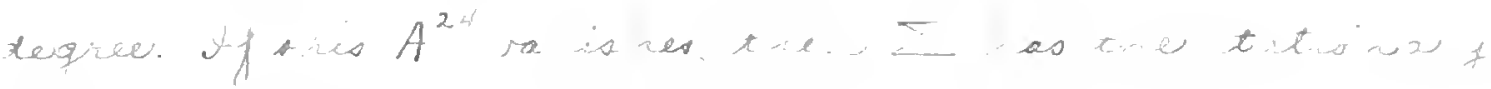

in as to ne is. is.

* is

Proc. Mat. Ac. Sci., Vul. 3, f $+49(19,7)$ 

Sita

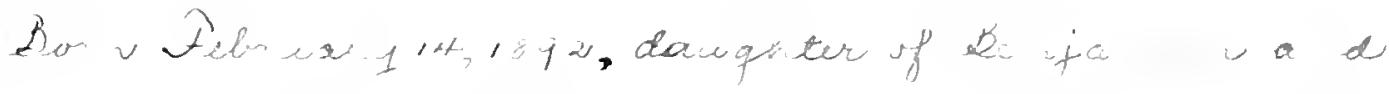

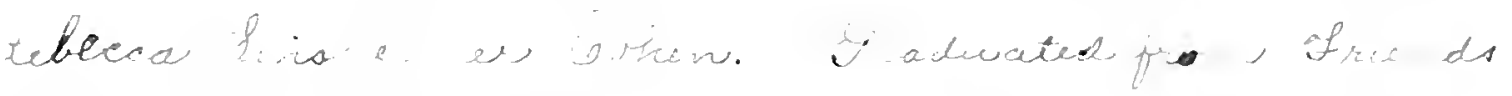

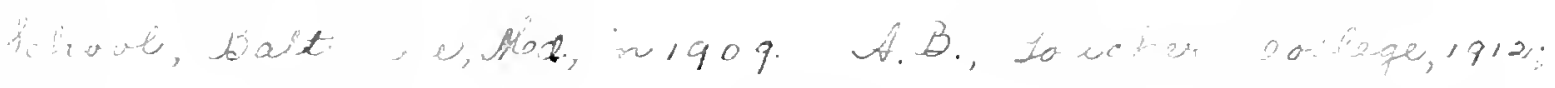

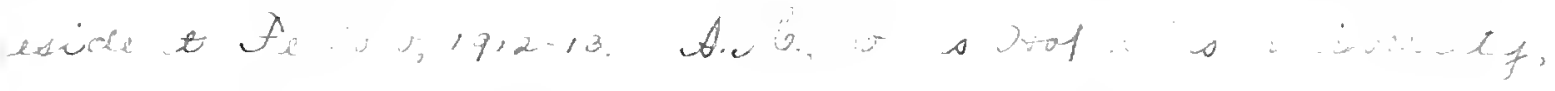

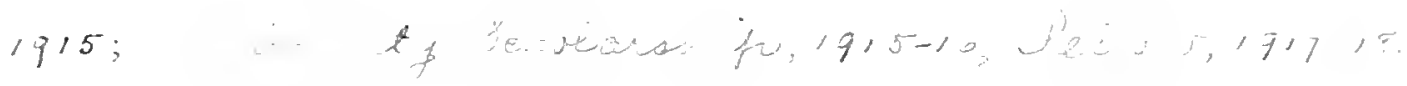

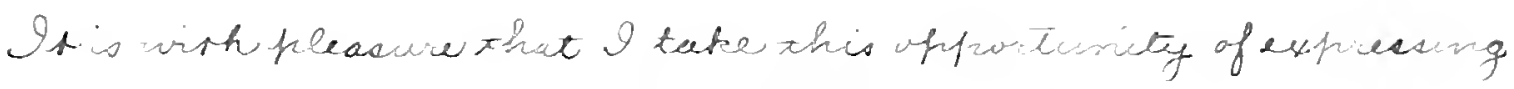

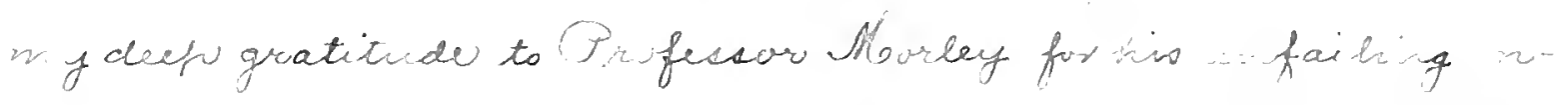

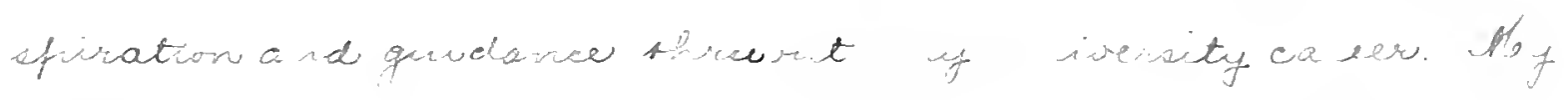

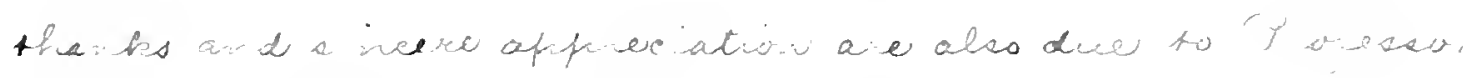

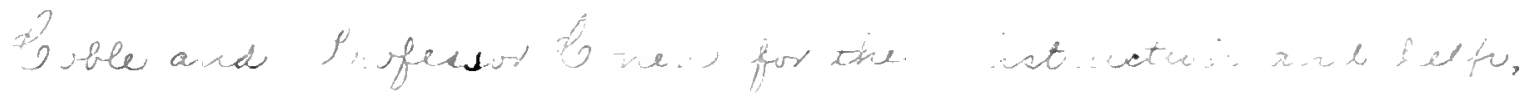
a to Profesear Bacen and Profesear Leviso of Swecher

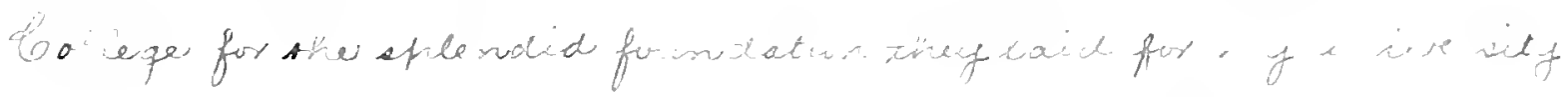

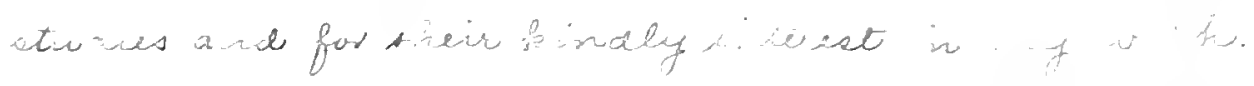

Werea in ine 







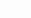


